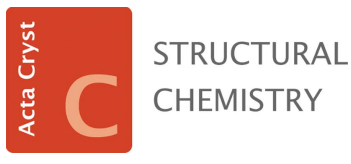

ISSN 2053-2296

Received 17 May 2018

Accepted 11 July 2018

Edited by J. R. Galán-Mascarós, Institute of Chemical Research of Catalonia (ICIQ), Spain

Keywords: polyoxometalate; POM; isopolytungstate; three-dimensional structure; crystal structure; dodecatungstate; IPOT.

CCDC references: $1843518 ; 1843519$

Supporting information: this article has supporting information at journals.iucr.org/C

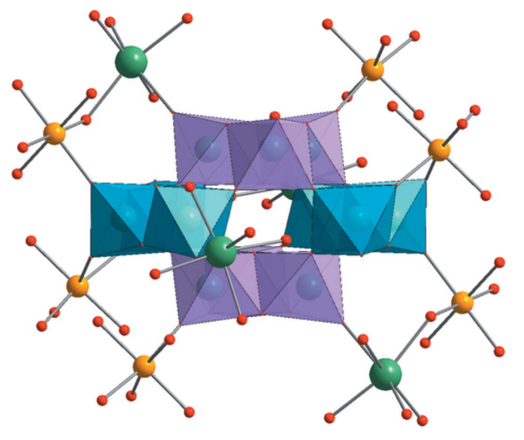

OPEN $\odot$ ACCESS

\section{Iron(II) and copper(II) paratungstates B: a single- crystal X-ray diffraction study}

\author{
Nadiia I. Gumerova, ${ }^{a}$ Anatolie Dobrov, ${ }^{a}$ Alexander Roller ${ }^{\mathrm{b}}$ and Annette Rompel ${ }^{\mathrm{a} *}$ \\ a'Universität Wien, Fakultät für Chemie, Institut für Biophysikalische Chemie, Althanstrasse 14, Wien 1090, Austria, and \\ bUniversität Wien, Fakultät für Chemie, Zentrum für Röntgenstrukturanalyse, Währinger Strasse 42, Wien 1090, Austria. \\ *Correspondence e-mail: annette.rompel@univie.ac.at
}

Paratungstate $\mathrm{B}$ is a common isopolytungstate (IPOT) built of the $\left[\mathrm{W}_{12} \mathrm{O}_{40}(\mathrm{OH})_{2}\right]^{10-}$ anion and exhibits a cluster-like construction of $12 \mathrm{~W}$ centred distorted octahedra. Due to a high surface charge density, the paratungstate anion acts as a multidentate ligand forming high-dimensional extended structures, which exhibit unique catalytic and magnetic properties. Two new paradodecatungstate $\mathrm{B}$ compounds decorated by iron(II) or copper(II), namely $\mathrm{Na}_{5} \mathrm{Fe}_{2.5}\left[\mathrm{~W}_{12} \mathrm{O}_{40}(\mathrm{OH})_{2}\right] \cdot 36 \mathrm{H}_{2} \mathrm{O} \quad\left(\mathbf{N a}_{5} \mathbf{F e}_{2.5}\right.$ paraB $)$ and $\mathrm{Na}_{4} \mathrm{Cu}_{3}\left[\mathrm{~W}_{12} \mathrm{O}_{40}(\mathrm{OH})_{2}\right] \cdot 28 \mathrm{H}_{2} \mathrm{O}\left(\mathbf{N a}_{4} \mathbf{C u}_{3}\right.$ paraB $)$, have been synthesized by a convenient aqueous solution method, and structurally characterized by singlecrystal and powder X-ray diffraction, IR spectroscopy, elemental analysis and thermogravimetric analysis. Both compounds crystallize in the triclinic $P \overline{1}$ space group. In both compounds, the $\left[\mathrm{W}_{12} \mathrm{O}_{40}(\mathrm{OH})_{2}\right]^{10-}$ polyanion acts as a multidentate ligand that links transition-metal and sodium cations, forming a three-dimensional framework.

\section{Introduction}

The structural diversity of polyoxometalates (Pope, 1983) and their proven applications in catalysis (Wang \& Yang, 2015), nanotechnology (Yamase \& Pope, 2002), electrochemistry (Sadakane \& Steckhan, 1998), materials science (Proust et al., 2008), molecular magnetism (Clemente-Juan et al., 2012), macromolecular crystallography (Bijelic \& Rompel, 2015, 2017; Molitor et al., 2017) and medicine (Fu et al., 2015; Bijelic et al., 2018a,b) have encouraged the synthesis of novel polyanions with promising properties. One of the most common isopolytungstates (IPOTs) is paratungstate $\mathrm{B}$, built of the $\left[\mathrm{W}_{12} \mathrm{O}_{40}(\mathrm{OH})_{2}\right]^{10-}$ anion that is stable in aqueous acidic solution and exhibits a cluster-like construction of $12 \mathrm{~W}$-centred distorted octahedra (Evans \& Rollins, 1976; Pope, 1983). Due to a high surface charge density, the paratungstate anion acts as a multidentate ligand, which can coordinate alkaline (Peresypkina et al., 2014) and transition-metal cations (Radio et al., 2010, 2011; Gumerova et al., 2015), and also act as a precursor (Sokolov et al., 2012). By coordinating transitionmetal cations, paratungstates can form high-dimensional extended structures, which exhibit unique catalytic (He et al., 2008; Chen et al., 2017) and magnetic properties ( $\mathrm{Li}$ et al., 2008, 2009). So far, three paratungstates $\mathrm{B}$ with $\mathrm{Fe}^{\mathrm{II}}$ and nine with $\mathrm{Cu}^{\mathrm{II}}$ as counter-cations have been successfully synthesized and characterized by X-ray diffraction (Table 1). We present herein two novel paratungstates $\mathrm{B}$, one with $\mathrm{Fe}^{\mathrm{II}}$ and one with $\mathrm{Cu}^{\mathrm{II}}$, namely the double sodium-iron(II) paratungstate $\mathrm{Na}_{5} \mathrm{Fe}_{2.5}\left[\mathrm{~W}_{12} \mathrm{O}_{40}(\mathrm{OH})_{2}\right] \cdot 36 \mathrm{H}_{2} \mathrm{O}$ (denoted $\mathbf{N a}_{5} \mathbf{F e}_{2.5^{-}}$ paraB) and the double sodium-copper(II) paratungstate $\mathrm{Na}_{4} \mathrm{Cu}_{3}\left[\mathrm{~W}_{12} \mathrm{O}_{40}(\mathrm{OH})_{2}\right] \cdot 28 \mathrm{H}_{2} \mathrm{O}$ (denoted $\mathbf{N a}_{4} \mathbf{C u}_{3}$ paraB), 
Table 1

$\mathrm{Fe}^{\mathrm{II}}$ - and $\mathrm{Cu}^{\mathrm{II}}$-containing paratungstates B [based on the Inorganic Crystal Structure Database (FIZ, Karlsruhe; http://www.fiz-informationsdienste.de/ DB/icsd/www-recherche.html) and the Cambridge Structural Database (CSD; Groom et al., 2016)].

\begin{tabular}{|c|c|c|c|c|}
\hline Compounds & $\begin{array}{l}\text { Unit-cell parameters } a, b \text { and } c(\AA) \text {, } \\
\text { and } \alpha, \beta \text { and } \gamma\left({ }^{\circ}\right)\end{array}$ & $\begin{array}{l}\text { Volume }\left(\AA^{3}\right), Z \text { and } \\
\text { space group }\end{array}$ & $\begin{array}{l}\text { Synthesis details } \\
\text { (source of } \mathrm{W} ; \mathrm{W}: M^{\mathrm{II}} \\
\text { ratio, with } M=\mathrm{Fe} \text {, } \\
\mathrm{Cu} ; \mathrm{pH} \text { ) }\end{array}$ & Reference \\
\hline \multicolumn{5}{|l|}{$\mathrm{Fe}^{\mathrm{II}}$} \\
\hline $\mathrm{K}_{6}\left[\left\{\mathrm{Fe}\left(\mathrm{H}_{2} \mathrm{O}\right)_{4}\right\}_{2}\left(\mathrm{H}_{2} \mathrm{~W}_{12} \mathrm{O}_{42}\right)\right] \cdot 15 \mathrm{H}_{2} \mathrm{O}$ & $\begin{array}{l}\text { 14.9967 (5), } 10.3872(3), 18.8237(6) \\
\quad 90,93.407(1), 90\end{array}$ & $2927.1(2), 2, P 2_{1} / n$ & $\mathrm{~K}_{2} \mathrm{WO}_{4} ; 12: 1.4 ;-$ & Yang et al. (2003) \\
\hline$\left(\mathrm{H}_{3} \mathrm{O}\right)_{2}\left[\left\{\mathrm{Fe}\left(\mathrm{H}_{2} \mathrm{O}\right)_{4} \mathrm{Fe}\left(\mathrm{H}_{2} \mathrm{O}\right)_{3}\right\}_{2}\left(\mathrm{H}_{2} \mathrm{~W}_{12} \mathrm{O}_{42}\right)\right] \cdot 20 \mathrm{H}_{2} \mathrm{O}$ & $\begin{array}{l}12.1794(4), 22.4938(4), 11.6941(3) \\
\quad 90,105.731(2), 90\end{array}$ & $3083.7(1), 2, P 2_{1} / c$ & $\mathrm{Li}_{2} \mathrm{WO}_{4} ; 12: 1.4 ;-$ & Yang et al. (2003) \\
\hline $\mathrm{Na}_{5}\left[\left\{\mathrm{Fe}\left(\mathrm{H}_{2} \mathrm{O}\right)_{3}\right\}_{2}\left\{\mathrm{Fe}\left(\mathrm{H}_{2} \mathrm{O}\right)_{4}\right\}_{0.5}\left(\mathrm{H}_{2} \mathrm{~W}_{12} \mathrm{O}_{42}\right)\right] \cdot 30 \mathrm{H}_{2} \mathrm{O}$ & $\begin{array}{l}12.121(2), 12.426(3), 13.247(3) \\
\quad 68.33(3), 71.33(3), 71.44(3)\end{array}$ & 1710.7 (6), $1, P \overline{1}$ & $\mathrm{Na}_{2} \mathrm{WO}_{4} ; 12: 1.4 ;-$ & Yang et al. (2003) \\
\hline \multicolumn{5}{|l|}{$\mathrm{Cu}^{\mathrm{II}}$} \\
\hline $\mathrm{Na}_{8}\left[\mathrm{Cu}\left(\mathrm{H}_{2} \mathrm{O}\right)_{2}\left(\mathrm{H}_{2} \mathrm{~W}_{12} \mathrm{O}_{42}\right)\right] \cdot 30 \mathrm{H}_{2} \mathrm{O}$ & $\begin{array}{l}13.081(4), 13.160(6), 20.127(6) \\
\quad 78.294(12), 78.524(11), 72.593(11)\end{array}$ & $3201.7(17), 2, P \overline{1}$ & $\mathrm{Na}_{2} \mathrm{WO}_{4} ; 12: 2.4 ; 4.8$ & Li et al. (2008) \\
\hline $\mathrm{KNa}_{3}\left[\mathrm{Cu}\left(\mathrm{H}_{2} \mathrm{O}\right)_{2}\left\{\mathrm{Cu}\left(\mathrm{H}_{2} \mathrm{O}\right)_{3}\right\}_{2}\left(\mathrm{H}_{2} \mathrm{~W}_{12} \mathrm{O}_{42}\right)\right] \cdot 16 \mathrm{H}_{2} \mathrm{O}$ & $\begin{array}{l}10.799(2), 11.914(2), 13.377(3) \\
\quad 70.18(3), 68.07(3), 64.80(3)\end{array}$ & $1410.9(5), 1, P \overline{1}$ & $\begin{array}{l}\mathrm{Na}_{2}\left[\mathrm{~W}_{12} \mathrm{O}_{40}(\mathrm{OH})_{2}\right] \\
\quad 12: 2 ; 3.5\end{array}$ & Li et al. (2009) \\
\hline $\begin{array}{c}{\left[\left\{\mathrm{Na}_{2}\left(\mu-\mathrm{H}_{2} \mathrm{O}\right)_{2}\left(\mathrm{H}_{2} \mathrm{O}\right)_{6}\right\}\left\{\mathrm{Cu}\left(\mathrm{H}_{2} \mathrm{O}\right)_{2}\right\}\left\{\mathrm{Cu}\left(\mathrm{H}_{2} \mathrm{O}\right)_{4}\right\}_{2}-\right.} \\
\left.\left\{\mathrm{Cu}_{2}(\mu-\mathrm{OH})_{2}\left(\mathrm{H}_{2} \mathrm{O}\right)_{6}\right\}\left(\mathrm{H}_{2} \mathrm{~W}_{12} \mathrm{O}_{42}\right)\right] \cdot 10 \mathrm{H}_{2} \mathrm{O}\end{array}$ & $\begin{array}{l}10.697(5), 12.921(5), 13.653(5) \\
\quad 73.608(5), 75.671(5), 67.748(5)\end{array}$ & $1654.4(12), 1, P \overline{1}$ & $\begin{array}{l}\left(\mathrm{NH}_{4}\right)_{6}\left[\mathrm{~W}_{12} \mathrm{O}_{40}\right] \\
12: 0.4 ; 6.2\end{array}$ & Kong et al. (2010) \\
\hline $\begin{array}{l}{\left[\left\{\mathrm{Na}_{(}\left(\mathrm{H}_{2} \mathrm{O}\right)_{4}\right\}_{2}\left\{\mathrm{Cu}_{0.5}\left(\mathrm{H}_{2} \mathrm{O}\right)\right\}_{4}\left\{\mathrm{Cu}_{0.5}\left(\mathrm{H}_{2} \mathrm{O}\right)_{1.5}\right\}_{2^{-}}\right.} \\
\left.\quad\left(\mathrm{H}_{4} \mathrm{~W}_{12} \mathrm{O}_{42}\right)\right] \cdot 3 \mathrm{H}_{2} \mathrm{O}\end{array}$ & $\begin{array}{l}10.7060(11), 12.7124(14), 13.1664(14) ; \\
\quad 113.7600(10), 90.8230(10), 111.8290(10)\end{array}$ & $1493.8(3), 1, P \overline{1}$ & $\mathrm{Na}_{2} \mathrm{WO}_{4} ; 12: 3 ; 6.5$ & Gao et al. (2011) \\
\hline$\left[\mathrm{Na}_{2}\left(\mathrm{H}_{2} \mathrm{O}\right)_{10}\right]\left[\mathrm{Cu}_{4}\left(\mathrm{H}_{2} \mathrm{O}\right)_{12}\left(\mathrm{H}_{2} \mathrm{~W}_{12} \mathrm{O}_{42}\right)\right] \cdot 15 \mathrm{H}_{2} \mathrm{O}$ & $\begin{array}{l}10.1535(2), 13.2118(3), 13.7049(5) \\
\quad 112.692(3), 94.771(3), 102.969(2)\end{array}$ & $1623.15(8), 1, P \overline{1}$ & $\mathrm{Na}_{2} \mathrm{WO}_{4} ; 12: 36 ; 4$ & Qu et al. (2012) \\
\hline $\mathrm{Cu}_{3}\left(\mathrm{H}_{2} \mathrm{O}\right)_{8}\left[\mathrm{H}_{6} \mathrm{~W}_{12} \mathrm{O}_{42}\right]$ & $\begin{array}{l}10.6753(5), 12.7814(5), 13.0976(5) \\
113.737(4), 90.433(3), 112.560(4)\end{array}$ & $1482.73(12), 2, P \overline{1}$ & $\begin{array}{l}\left(\mathrm{NH}_{4}\right)_{6}\left[\mathrm{~W}_{12} \mathrm{O}_{40}\right] \\
12: 36 ;-\end{array}$ & Chen et al. (2017) \\
\hline$\left(\mathrm{NH}_{4}\right)_{8}\left[\mathrm{Cu}\left(\mathrm{H}_{2} \mathrm{O}\right)_{2} \mathrm{H}_{2} \mathrm{~W}_{12} \mathrm{O}_{42}\right] \cdot 10 \mathrm{H}_{2} \mathrm{O}$ & $\begin{array}{l}14.278(5), 15.435(5), 24.881(5) \\
\quad 90,90,90\end{array}$ & $5483(3), 2, P b c n$ & $\begin{array}{l}\left(\mathrm{NH}_{4}\right)_{6}\left[\mathrm{~W}_{12} \mathrm{O}_{40}\right] \\
12: 2.5 ; 4.8\end{array}$ & Zhang (2012) \\
\hline $\mathrm{Na}_{2} \mathrm{Cu}_{3}(\mathrm{CuOH})_{2}\left[\mathrm{~W}_{12} \mathrm{O}_{40}(\mathrm{OH})_{2}\right] \cdot 32 \mathrm{H}_{2} \mathrm{O}$ & $\begin{array}{l}10.6836(4), 12.9066(6), 13.6475(5) \\
\quad 73.561(4), 75.685(3), 67.666(4)\end{array}$ & $1648.68(12), 1, P \overline{1}$ & $\mathrm{Na}_{2} \mathrm{WO}_{4} ; 12: 7.5 ;-$ & Radio et al. (2014) \\
\hline $\mathrm{Na}_{2} \mathrm{Cu}_{5}\left(\mathrm{H}_{2} \mathrm{O}\right)_{24}(\mathrm{OH})_{2}\left[\mathrm{H}_{2} \mathrm{~W}_{12} \mathrm{O}_{42}\right] \cdot 10 \mathrm{H}_{2} \mathrm{O}$ & $\begin{array}{l}10.7140(8), 12.9476(9), 13.6696(10) \\
\quad 73.56,75.73,67.69\end{array}$ & $1661.8(2), 1, P \overline{1}$ & $\mathrm{Na}_{2} \mathrm{WO}_{4} ; 12: 20 ; 3.8$ & Qu et al. (2015) \\
\hline
\end{tabular}

Table 2

Experimental details.

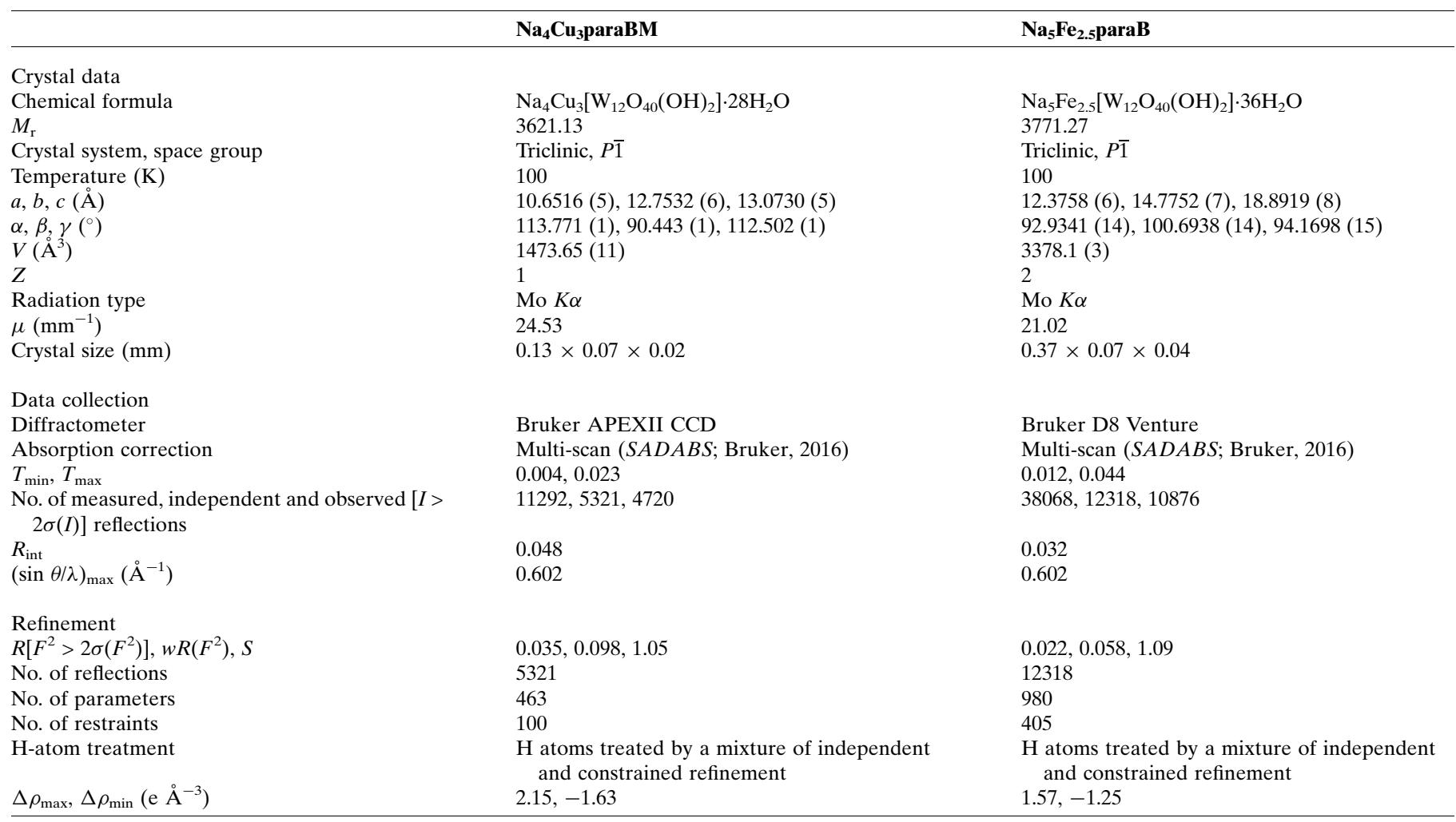

Computer programs: APEX3 (Bruker, 2015), SAINT (Bruker, 2015), SHELXS97 (Sheldrick, 2008), shelXle (Hübschle et al., 2011), SHELXL2014 (Sheldrick, 2015), SHELXL2016 (Sheldrick, 2015), OLEX2 (Dolomanov et al., 2009) and DIAMOND (Brandenburg, 2006). 
which were synthesized by a convenient aqueous solution method.

\section{Experimental}

\subsection{Synthesis and crystallization}

The reagents were used as purchased from Sigma-Aldrich without further purification.

2.1.1. Synthesis of $\mathrm{Na}_{5} \mathrm{Fe}_{2.5}$ paraB. Iron powder $(0.112 \mathrm{~g}$, $2 \mathrm{mmol}$ ) was added to a solution $(15 \mathrm{ml})$ of $\mathrm{Na}_{2} \mathrm{WO}_{4} \cdot 2 \mathrm{H}_{2} \mathrm{O}$ (3.96 g, $12 \mathrm{mmol}$ ), which was acidified to $\mathrm{pH}=2.5$ with $\mathrm{HCl}$ $(1 M)$. The mixture was stirred in an ultrasonic bath, giving a deep-blue solution, which was left to stand closed at room temperature. The pale-red-brown crystals which grew on the beaker walls were collected after three weeks (yield $\sim 2 \mathrm{~g}$, $\sim 53 \%$, based on $\mathrm{W}$ ). Elemental analysis found (calculated) for $\mathrm{Fe}_{2.5} \mathrm{H}_{74} \mathrm{Na}_{5} \mathrm{O}_{78} \mathrm{~W}_{12}$ (\%): Na 3.13 (3.03), Fe 3.71 (3.69), W 56.8 (58.32).

2.1.2. Synthesis of $\mathrm{Na}_{4} \mathrm{Cu}_{3}$ paraB. Sodium orthotungstate $\mathrm{Na}_{2} \mathrm{WO}_{4} \cdot 2 \mathrm{H}_{2} \mathrm{O}(5.5 \mathrm{~g}, 16.7 \mathrm{mmol})$ was dissolved in water
( $25 \mathrm{ml}$ ) and the $\mathrm{pH}$ was adjusted to 8 by adding dilute $\mathrm{HNO}_{3}$ $(1 M)$. An aqueous solution $(10 \mathrm{ml})$ of $\mathrm{Cu}\left(\mathrm{NO}_{3}\right)_{2} \cdot 3 \mathrm{H}_{2} \mathrm{O}(0.5 \mathrm{~g}$, $2.1 \mathrm{mmol}$ ) was then added dropwise, while the $\mathrm{pH}$ was maintained between 3.0 and 4.5 with $\mathrm{HNO}_{3}(1 M)$. The final mixture was filtered $(\mathrm{pH}=4.2)$ and allowed to stand closed at room temperature. Light-blue crystals formed within two months (yield $\sim 3.5 \mathrm{~g}, 69 \%$ based on $\mathrm{W}$ ). Elemental analysis found (calculated) for $\mathrm{Cu}_{3} \mathrm{H}_{58} \mathrm{Na}_{4} \mathrm{O}_{70} \mathrm{~W}_{12}$ (\%): $\mathrm{Na} 2.62$ (2.51), Cu 5.13 (5.20), W 59.1 (60.16).

\subsection{IR spectroscopy}

The title compounds were identified by IR measurements on a Bruker Vertex70 IR Spectrometer equipped with a single-reflection diamond-ATR unit (ATR is attenuated total reflectance) in the range $4000-400 \mathrm{~cm}^{-1}$.

\subsection{TGA measurements}

Thermogravimetric analysis (TGA) was performed on a Mettler SDTA851e Thermogravimetric Analyzer under a

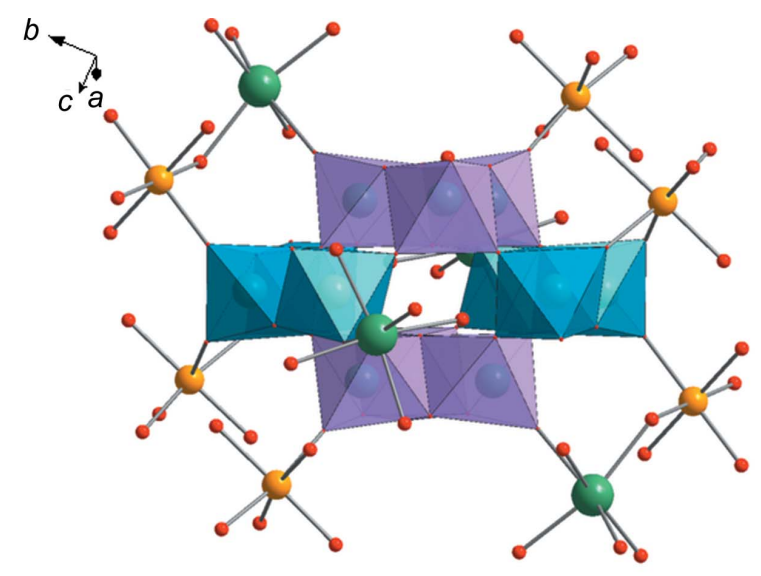

(a)

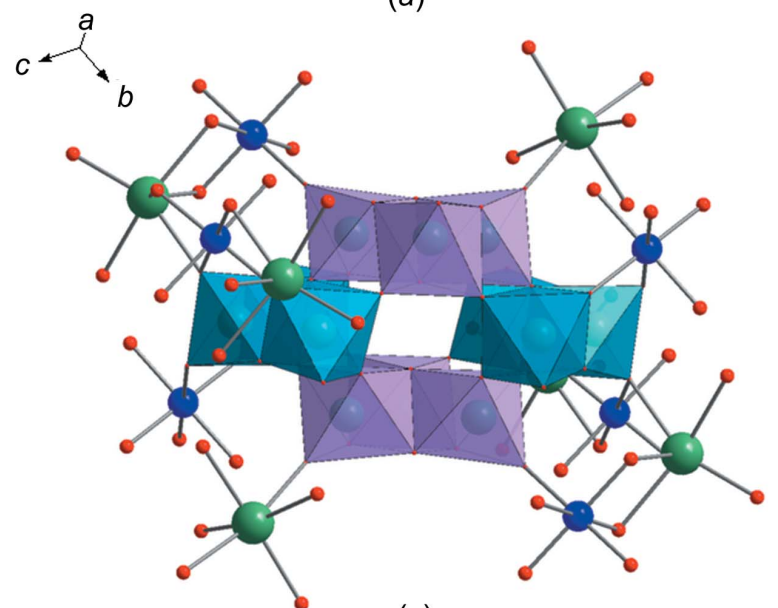

(c)

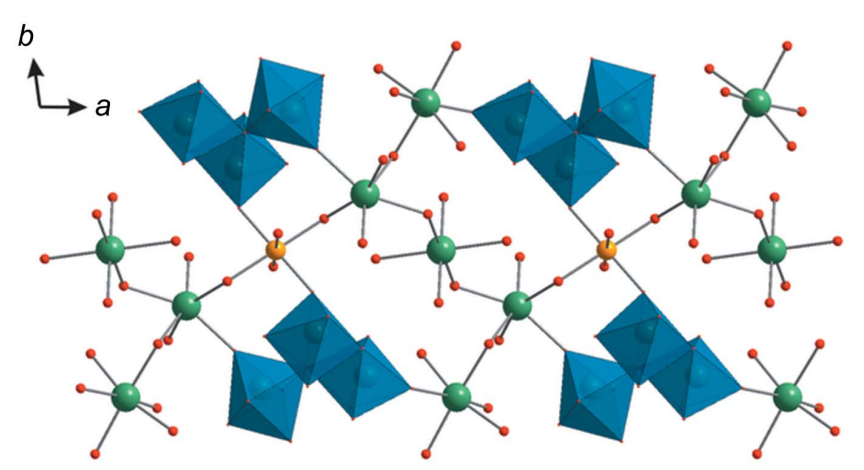

(b)

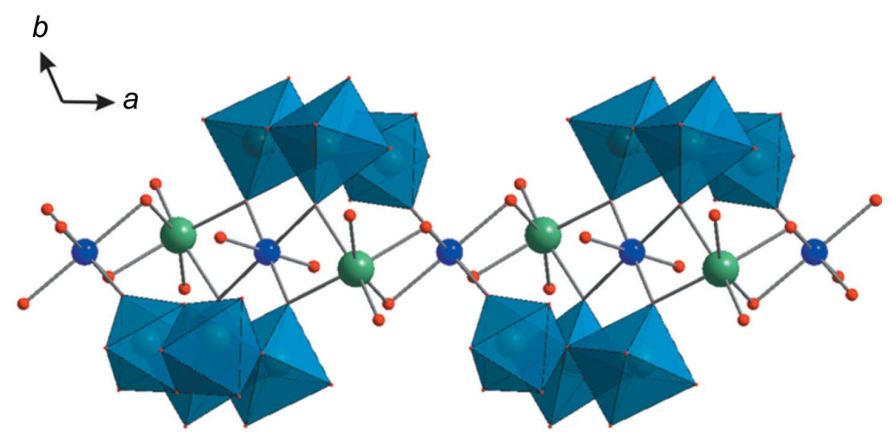

(d)

Figure 1

Structural elements in $\mathbf{N a}_{5} \mathbf{F e}_{2.5}$ paraB and $\mathbf{N a}_{4} \mathbf{C u} \mathbf{u}_{3}$ paraB. (a) The $\left[\mathrm{W}_{12} \mathrm{O}_{40}(\mathrm{OH})_{2}\right]^{10-}$ anion in $\mathbf{N a}_{5} \mathbf{F e}_{2.5}$ paraB connected to four Na ${ }^{+}$and six Fe ${ }^{2+}$ ions via terminal $\mathrm{O}$ atoms. (b) A fragment of the infinite $1 \mathrm{D}$ chain in $\mathbf{N a}_{5} \mathbf{F e}_{2.5}$ paraB consisting of $\mathrm{Na}$ and Fe polyhedra. $(c)$ The $\left[\mathrm{W}_{12} \mathrm{O}_{40}(\mathrm{OH})_{2}\right]^{10-}$ anion in $\mathrm{Na}_{4} \mathrm{Cu}_{3}$ paraB connected to six $\mathrm{Na}^{+}$and six $\mathrm{Cu}^{2+}$ ions via terminal $\mathrm{O}$ atoms. (d) A fragment of the infinite $1 \mathrm{D}$ chain in $\mathbf{N a} \mathbf{a}_{4} \mathrm{Cu}_{3} \mathbf{p a r a B}$ consisting of Na and $\mathrm{Cu}$ polyhedra. Colour code: $\left\{\mathrm{WO}_{6}\right\}$ are light-blue or violet octahedra, $\left\{\mathrm{W}_{3} \mathrm{O}_{14}\right\}$ are blue octahedra and $\left\{\mathrm{W}_{3} \mathrm{O}_{13}\right\}$ are violet octahedra, and $\mathrm{Na}$ atoms are green, $\mathrm{Fe}$ orange, $\mathrm{Cu}$ blue and $\mathrm{O}$ red. 
Figure 2

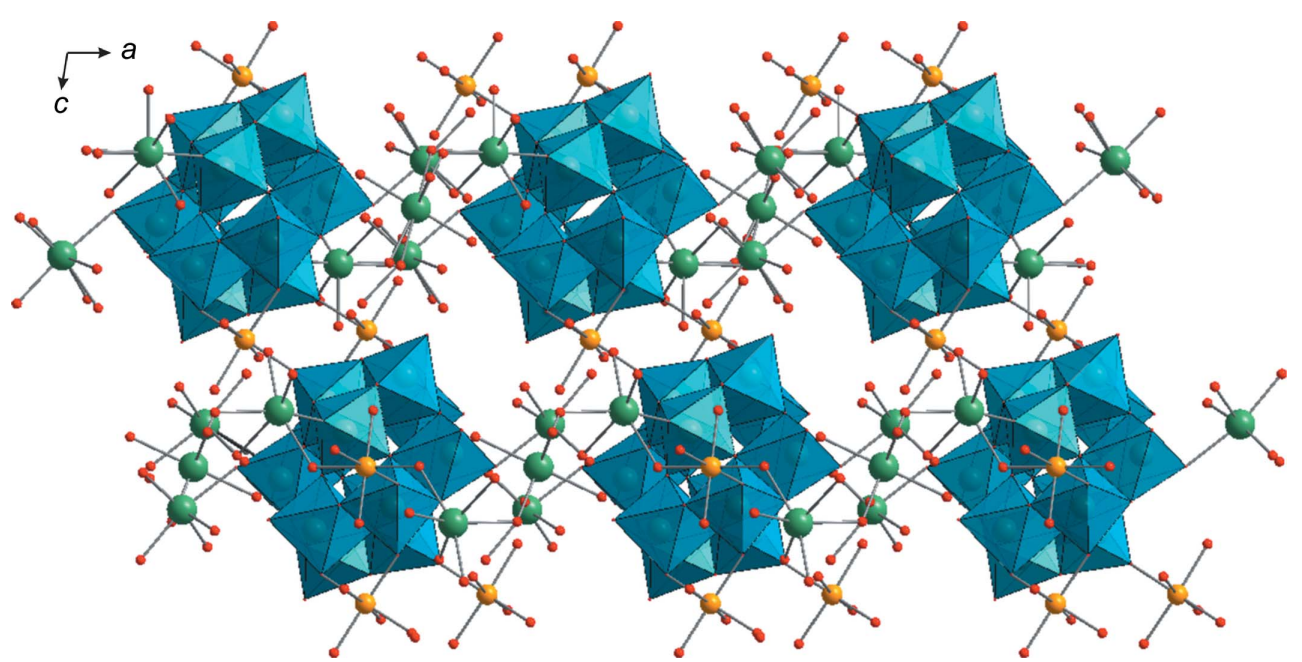

The crystal packing of $\mathbf{N a}_{5} \mathbf{F e}_{2.5}$ paraB, viewed along the $b$ axis. Colour code: $\left\{\mathrm{WO}_{6}\right\}$ are light-blue octahedra and Na atoms are green, Fe yellow and $\mathrm{O}$ red.

nitrogen flow with a heating rate of $5 \mathrm{~K} \mathrm{~min}^{-1}$ in the region from 298 to $973 \mathrm{~K}$.

\subsection{Elemental analysis}

Elemental analysis was conducted using inductive-coupled plasma-mass spectrometry (PerkinElmer Elan 6000 ICP MS) and atomic absorption spectroscopy (PerkinElmer 1100 Flame AAS) in aqueous solutions containing $2 \% \mathrm{HNO}_{3}$. Standards were prepared from single-element standard solutions of concentration $1000 \mathrm{mg} \mathrm{l}^{-1}$ (from Merck, Ultra Scientific and Analytika Prague).

\subsection{Powder X-ray diffraction}

Powder X-ray diffraction (PXRD) was performed on a Bruker D8 Advance diffractometer, with $\mathrm{Cu} K \alpha$ radiation $(\lambda=$ $1.54056 \AA$ A), a Lynxeye silicon strip detector, a SolX energy dispersive detector and a variable slit aperture of $12 \mathrm{~mm}$. The $2 \theta$ range was $8-50^{\circ}$.

\subsection{Refinement}

In Table 2, the crystallographic characteristics of the two new paratungstates $\mathrm{B}$ and the experimental conditions of the data collection and refinement are reported. The positions of the independent $\mathrm{H}$ atoms were obtained by difference Fourier techniques and were refined with free isotropic displacement parameters.

Fixed isotropic displacement parameters for all $\mathrm{H}$ atoms with a value equal to $1.5 U_{\text {eq }}$ of the corresponding $\mathrm{O}-\mathrm{H}$ group atom were assigned. Restrained distances for $D-\mathrm{H}$ bonds were applied to avoid short $D-\mathrm{H} \cdots \mathrm{H}-D$ interactions. To force correct bonds, specified bonds were added to or removed from the connectivity list.

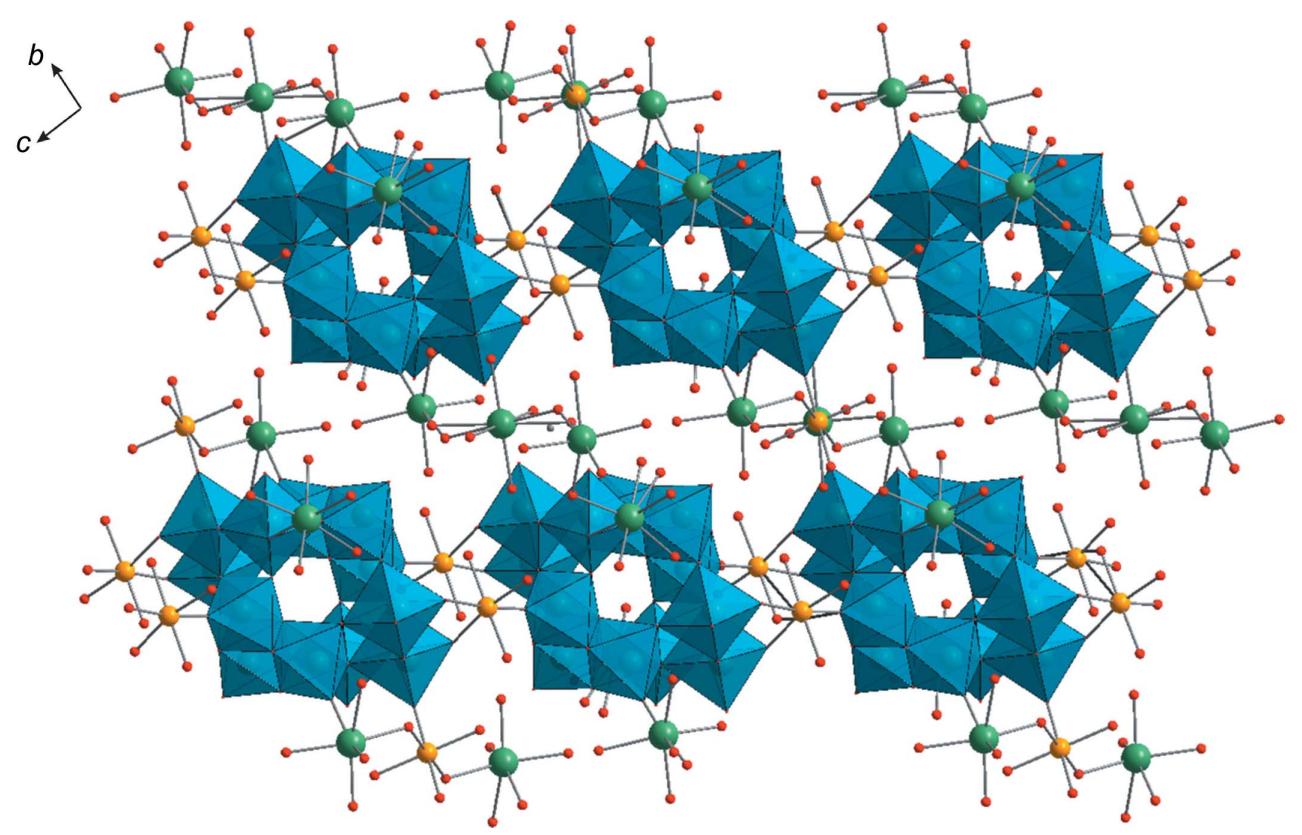

Figure 3

The crystal packing of $\mathbf{N a}_{5} \mathbf{F e}_{2.5}$ paraB, viewed along the $a$ axis. Colour code: $\left\{\mathrm{WO}_{6}\right\}$ are light-blue octahedra and $\mathrm{Na}$ atoms are green, Fe yellow and $\mathrm{O}$ red. 


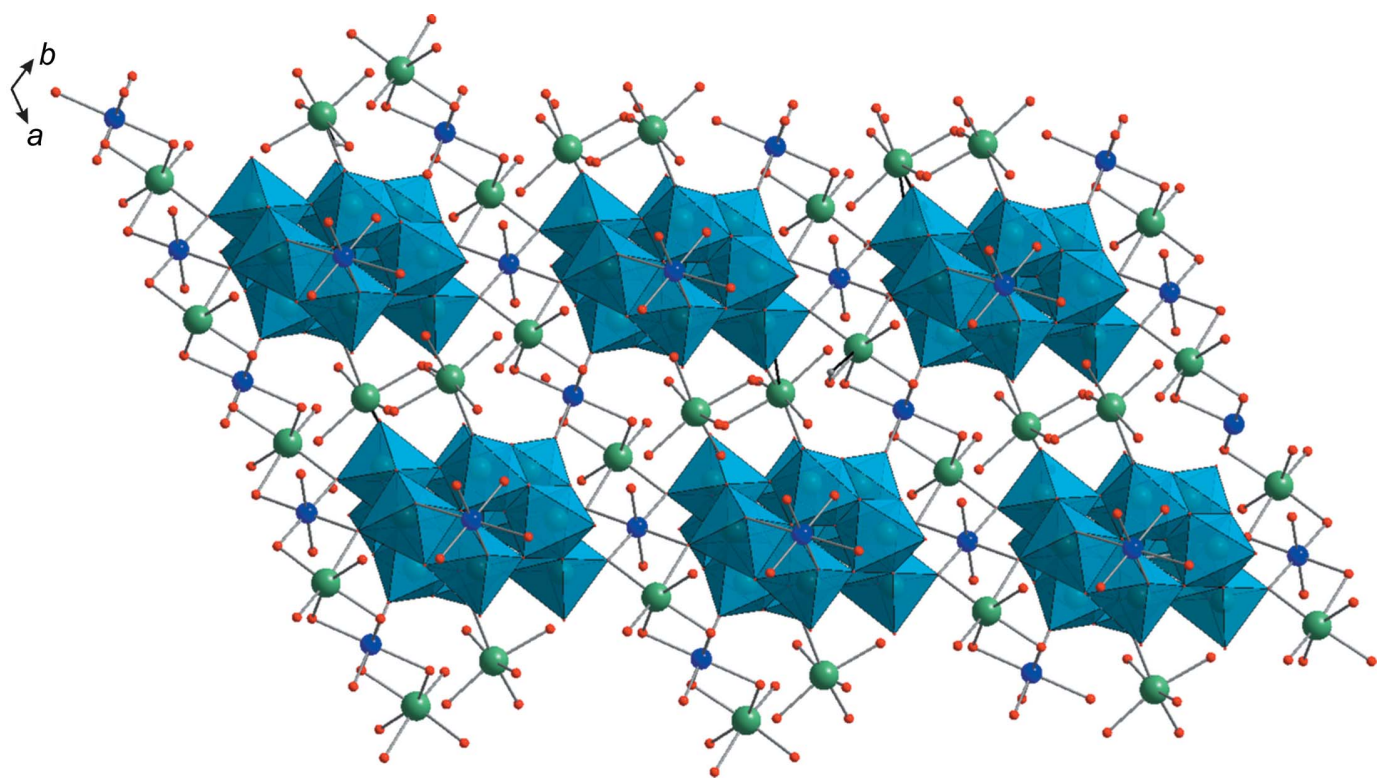

Figure 4

The crystal packing of $\mathbf{N a}_{4} \mathbf{C u}_{3}$ paraB, viewed along the $c$ axis. Colour code: $\left\{\mathrm{WO}_{6}\right\}$ are light-blue octahedra and $\mathrm{Na}$ atoms are green, $\mathrm{Cu}$ blue and $\mathrm{O}$ red.

The disordered water molecules in the coordination spheres of atom $\mathrm{Na} 1$ in $\mathbf{N a}_{\mathbf{4}} \mathbf{C u}_{3}$ paraB and of atoms $\mathrm{Na} 4$ and $\mathrm{Na} 5$ in $\mathbf{N a}_{5} \mathbf{F e}_{2.5}$ paraB were refined with two positions with fixed occupancy factors of 0.5 .

In $\mathbf{N a}_{4} \mathbf{C u}_{3}$ paraB, part of the disordered water molecules were not modelled and the disordered density was considered using the OLEX2 (Dolomanov et al., 2009) implementation of BYPASS (a.k.a. SQUEEZE; Spek, 2015). The modelled electron density is consistent with approximately four water molecules per unit cell.

The structures have been deposited with the Inorganic Crystal Structure Database (ICSD) (http://www2.fiz-karlsruhe.de/icsd_home.html) under collection numbers 434558 and 434559 .

\section{Results and discussion}

The syntheses of $\mathbf{N a}_{5} \mathbf{F e}_{2.5}$ paraB and $\mathbf{N a}_{\mathbf{4}} \mathbf{C u}_{3}$ paraB were carried out with $\mathrm{W}^{\mathrm{VI}}$-to- $M^{\mathrm{II}}$ ratios of $\mathrm{W}: \mathrm{Fe}=12: 2$ and $\mathrm{W}: \mathrm{Cu}=$ 12:1.5, and a $\mathrm{pH}$ of 2.5 for $\mathbf{N a}_{5} \mathbf{F e}_{2.5} \mathbf{p a r a B}$ and 4.2 for $\mathbf{N a}_{4} \mathbf{C u}_{3}$ paraB, which are different from previously reported conditions (Table 1) and made it possible to obtain compounds with new $\mathrm{Fe}-\mathrm{Na}$ and $\mathrm{Cu}-\mathrm{Na}$ compositions. The presence of $\mathrm{Na}^{\mathrm{I}}$ as counter-cation in paratungstates $\mathrm{B}$, together with $\mathrm{Cu}^{\mathrm{II}}$ or $\mathrm{Fe}^{\mathrm{II}}$, have been observed previously both in excess and in deficiency of the transition-metal ion in the reaction mixture, which had a $\mathrm{pH}$ in the range 3.5-6.5 (Table 1). This allows one to conclude that crystallization of paratungstates B as double-alkali-transition-metal salts is more preferable than crystallization of pure transition-metal paratungstates $\mathrm{B}$, regardless of the starting molar ratios of the components and the $\mathrm{pH}$ of the reaction system.

The main structural elements of $\mathbf{N a}_{5} \mathbf{F e}_{2.5}$ paraB and $\mathbf{N a}_{4} \mathbf{C u}_{3}$ paraB are shown in Fig. 1. Both compounds consist of paradodecatungstate $\mathrm{B}\left[\mathrm{W}_{12} \mathrm{O}_{40}(\mathrm{OH})_{2}\right]^{10-}$ polyanions (Evans
\& Rollins, 1976; Pope, 1983), sodium and transition-metal cations, and additional water molecules (Fig. 1). The paratungstate $B$ units observed in $\mathbf{N a}_{5} \mathbf{F e}_{2.5}$ paraB and $\mathbf{N a}_{4} \mathbf{C u}_{3^{-}}$ paraB are structurally identical to previously reported units (Table 1).

In $\mathbf{N a}_{\mathbf{4}} \mathbf{C} \mathbf{u}_{3}$ paraB, there is one-half unit of the POM, which lies on an inversion centre, in the asymmetric unit. For $\mathbf{N a}_{5} \mathbf{F e}_{2.5}$ paraB, there are two independent half-POM units in the asymmetric unit.

The centrosymmetric $\left[\mathrm{W}_{12} \mathrm{O}_{40}(\mathrm{OH})_{2}\right]^{10-}$ anion consists of four corner-sharing groups: two $\left\{\mathrm{W}_{3} \mathrm{O}_{13}\right\}$ (violet octahedra in Figs. $1 a$ and $1 c$ ) and two $\left\{\mathrm{W}_{3} \mathrm{O}_{14}\right\}$ (blue octahedra in Figs. $1 a$ and $1 c$ ) units. Each $\left\{\mathrm{W}_{3} \mathrm{O}_{13}\right\}$ fragment is formed by three edgesharing $\left\{\mathrm{WO}_{6}\right\}$ octahedra with a common $\mathrm{O}$ atom, while in the $\left\{\mathrm{W}_{3} \mathrm{O}_{14}\right\}$ triads, the three edge-sharing $\left\{\mathrm{WO}_{6}\right\}$ octahedra are linearly connected with no common $\mathrm{O}$ atom to the three $\mathrm{W}$ atoms. In the $\left\{\mathrm{W}_{3} \mathrm{O}_{13}\right\}$ groups, each octahedron has one terminal $\mathrm{O}$ atom, while in the $\left\{\mathrm{W}_{3} \mathrm{O}_{14}\right\}$ units, each octahedron has two unshared $\mathrm{O}$ atoms (Figs. $1 a$ and $1 c$ ). The $\mathrm{O}$ atoms

Table 3

Selected bond length and angles $\left(\AA,{ }^{\circ}\right)$ in $\mathbf{N a}_{5} \mathbf{F e}_{2.5}$ paraB and $\mathrm{Na}_{4} \mathrm{Cu}_{3}$ paraB

\begin{tabular}{lll}
\hline & $\mathbf{N a}_{5} \mathbf{F e}_{2.5} \mathbf{p a r a B}$ & $\mathbf{N a}_{\mathbf{4}} \mathbf{C} \mathbf{u}_{\mathbf{3}} \mathbf{p a r a} \mathbf{B}$ \\
\hline $\mathrm{W}=\mathrm{O}_{\mathrm{t}}$ & $1.719(4)-1.797(4)$ & $1.710(8)-1.780(7)$ \\
$\mathrm{W}-\mathrm{O}_{\mathrm{db} 1}$ & $1.888(4)-2.050(2)$ & $1.872(7)-2.103(7)$ \\
$\mathrm{W}-\mathrm{O}_{\mathrm{db} 2}$ & $1.826(3)-2.166(3)$ & $1.805(7)-2.098(7)$ \\
$\mathrm{W}-\mathrm{O}_{\mathrm{tb} 1}$ & $2.201(3)-2.297(3)$ & $2.207(7)-2.273(7)$ \\
$\mathrm{W}-\mathrm{O}_{\mathrm{tb} 2}$ & $1.895(4)-2.259(4)$ & $1.882(8)-2.287(7)$ \\
$\mathrm{W} \cdots \mathrm{W}$ (between & $3.649(4)-3.878(5)$ & $3.377(4)-3.688(2)$ \\
$\quad$ corner-sharing WO \\
$\mathrm{W} \cdots \mathrm{W}$ (between & & \\
$\quad$ edge-sharing $\left.\mathrm{WO}_{6}\right)$ & $3.273(4)-3.352(4)$ & $3.306(2)-3.377(3)$ \\
$M^{\mathrm{II}}-\mathrm{O}(M=\mathrm{Fe}$ or $\mathrm{Cu})$ & $2.087(4)-2.169(4)$ & $1.918(7)-2.366(8)$ \\
$\mathrm{Na}-\mathrm{O}$ & $2.302(11)-2.606(11)$ & $2.345(9)-2.519(13)$ \\
$\mathrm{O}-\mathrm{W}-\mathrm{O}$ & $70.73(14)-104.38(17)$ & $154.94(15)-177.56(16)$ \\
& $70.2(3)-105.6(3)$ & $152.8(3)-178.1(3)$ \\
\hline
\end{tabular}




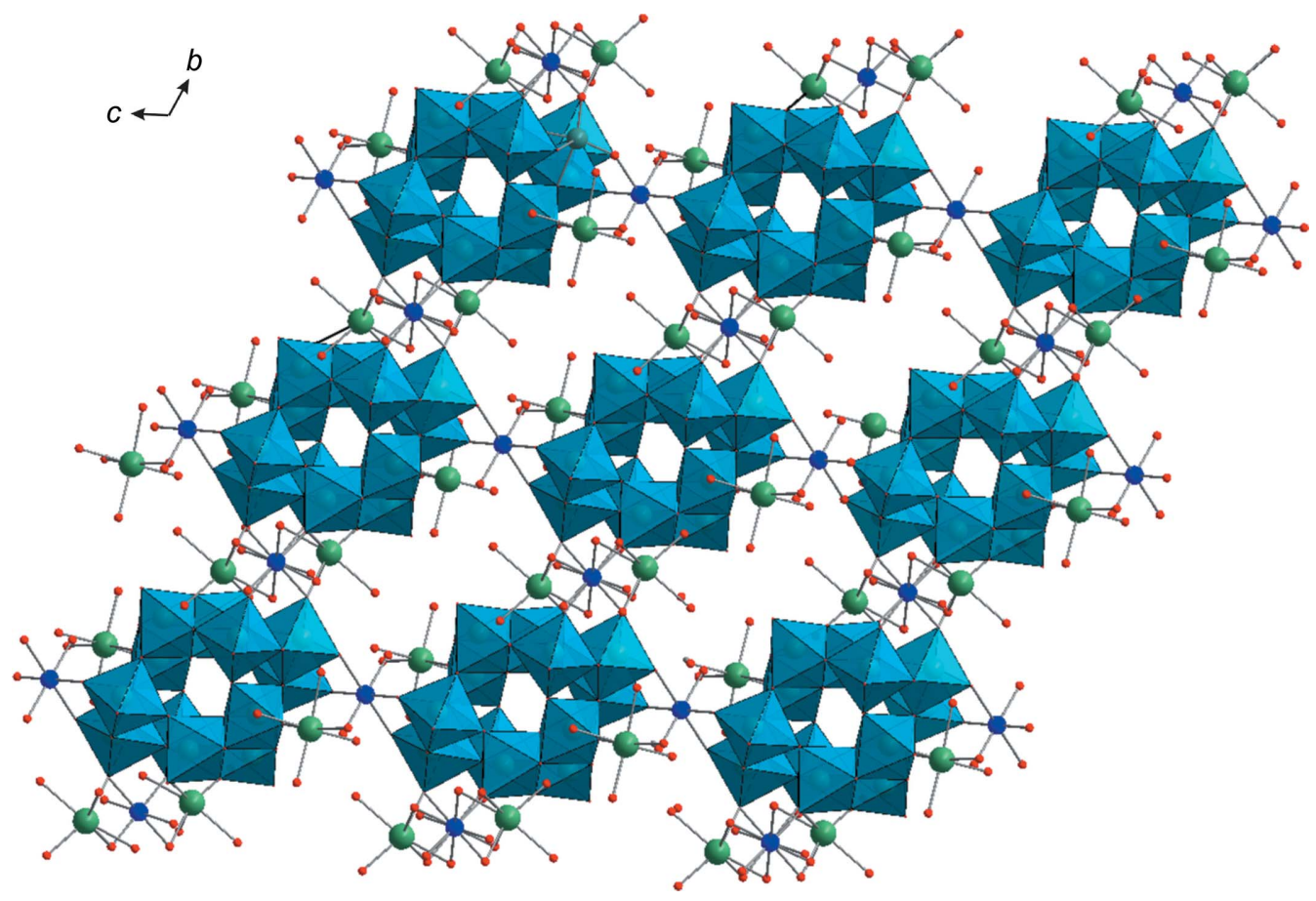

Figure 5

The crystal packing of $\mathbf{N a}_{4} \mathbf{C u}_{3}$ paraB, viewed along the $a$ axis. Colour code: $\left\{\mathrm{WO}_{6}\right\}$ are light-blue octahedra and Na atoms are green, $\mathrm{Cu}$ blue and $\mathrm{O}$ red.

connected to the $\mathrm{W}$ centres can be classified into three groups. The first group is comprised of terminal $\mathrm{O}$ atoms $\left(\mathrm{O}_{\mathrm{t}}\right)$, each bonded to one $\mathrm{W}$ atom. The second group consists of bridging $\mathrm{O}$ atoms $\left(\mathrm{O}_{\mathrm{db}}\right)$, each connected to two $\mathrm{W}$ atoms. There are two types of $\mathrm{O}_{\mathrm{db}}$, one bridges two $\mathrm{W}$ atoms within the same $\left\{\mathrm{W}_{3} \mathrm{O}_{13}\right\}$ or $\left\{\mathrm{W}_{3} \mathrm{O}_{14}\right\}$ fragment $\left(\mathrm{O}_{\mathrm{db} 1}\right)$, while the other bridges two $\mathrm{W}$ atoms between the different $\left\{\mathrm{W}_{3} \mathrm{O}_{13}\right\}$ and $\left\{\mathrm{W}_{3} \mathrm{O}_{14}\right\}$ units $\left(\mathrm{O}_{\mathrm{db} 2}\right)$. The third group contains triply bridging $\mathrm{O}$ atoms, linked by three $\mathrm{W}$ atoms. The triply bridging $\mathrm{O}$ atoms exclusively from $\left\{\mathrm{W}_{3} \mathrm{O}_{13}\right\}$ are labelled $\mathrm{O}_{\mathrm{tb} 1}$, whereas the $\mathrm{O}$ atoms bridging three $\mathrm{W}$ atoms between $\left\{\mathrm{W}_{3} \mathrm{O}_{13}\right\}$ and $\left\{\mathrm{W}_{3} \mathrm{O}_{14}\right\}$ units are labelled as $\mathrm{O}_{\mathrm{tb} 2}$.

The exact positions of the two protons in $\left[\mathrm{W}_{12} \mathrm{O}_{40}(\mathrm{OH})_{2}\right]^{10-}$ were located previously on triply bridging $\mathrm{O}$ atoms of $\left\{\mathrm{W}_{3} \mathrm{O}_{13}\right\}$ by neutron diffraction (Evans \& Prince, 1983). Selected bond

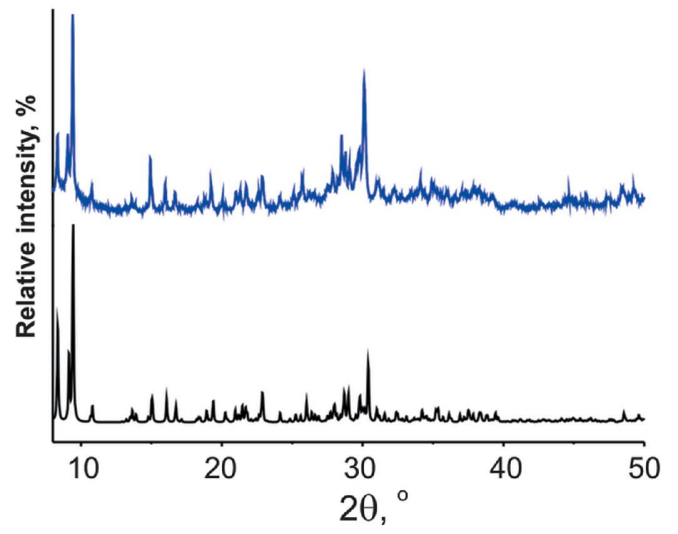

Figure 6

(a) lengths and angles are presented in Table 3. All the $\mathrm{W}$ atoms in $\left[\mathrm{W}_{12} \mathrm{O}_{40}(\mathrm{OH})_{2}\right]^{10-}$ exhibit the +VI oxidation state, when applying the bond valence sum (BVS) calculations of Brown \& Altermatt (1985). For $\mathbf{N a}_{5} \mathbf{F e}_{2.5}$ paraB and $\mathbf{N a}_{4} \mathbf{C u}_{3}$ paraB, we got average values of 6.01 and 6.09, respectively. BVS calculations for $\mathrm{Fe}$ and $\mathrm{Cu}$ sites show that both ions exhibit the $+\mathrm{II}$ oxidation state, with a value of 2.12 for $\mathrm{Fe}$ and 2.08 for $\mathrm{Cu}$.

In the crystal structure of $\mathbf{N a}_{\mathbf{5}} \mathbf{F e} \mathbf{2}_{\mathbf{2 . 5}}$ paraB, the paratungstate anions act as decadentate ligands, which are linked via terminal $\mathrm{O}$ atoms to six $\mathrm{Fe}^{2+}$ and four $\mathrm{Na}^{+}$cations. There are two crystallographically unique iron centres with different coordination modes (Figs. $1 a$ and $1 b$ ). The coordination sphere of one type of $\mathrm{Fe}^{2+}$ atom $(\mathrm{Fe} 2)$ is formed by two $\mathrm{O}_{\mathrm{t}}$ from the belt unit $\left\{\mathrm{W}_{3} \mathrm{O}_{14}\right\}$ of one $\left[\mathrm{W}_{12} \mathrm{O}_{40}(\mathrm{OH})_{2}\right]^{10-}$, one $\mathrm{O}_{t}$ from the capping $\left\{\mathrm{W}_{3} \mathrm{O}_{13}\right\}$ group of a neighbouring polyanion and

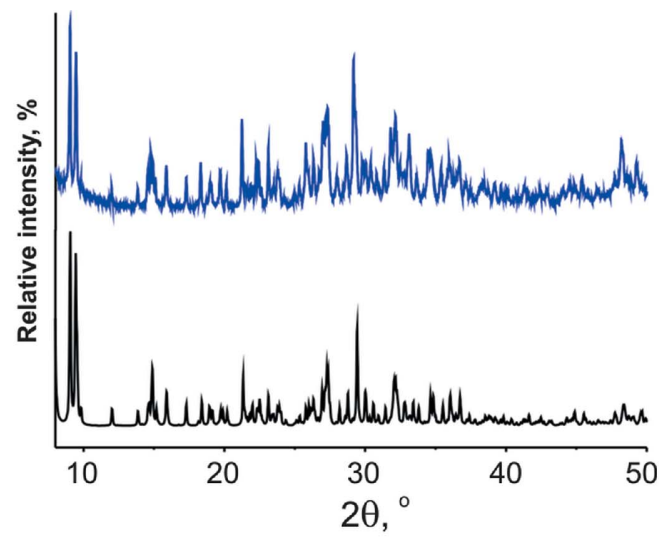

(b)

Experimental (blue) and simulated (black) X-ray diffraction patterns of (a) $\mathbf{N} \mathbf{a}_{4} \mathbf{C u}_{3}$ paraB and (b) $\mathbf{N a}_{5} \mathbf{F e}_{2.5} \mathbf{p a r a B}$. 
completed by three $\mathrm{H}_{2} \mathrm{O}$ molecules. The octahedrally coordinated second $\mathrm{Fe}^{2+}$ atom $(\mathrm{Fe} 1)$ is linked by two $\mathrm{O}_{\mathrm{t}}$ from the $\left\{\mathrm{W}_{3} \mathrm{O}_{13}\right\}$ units of two neighbouring polyanions, two $\mathrm{Na}^{+}$bridging $\mathrm{O}$ atoms and two lattice $\mathrm{H}_{2} \mathrm{O}$ molecules. The $\mathrm{Fe} 1$ octahedron and three $\mathrm{Na}\left(\mathrm{H}_{2} \mathrm{O}\right)_{6}$ units from the infinite onedimensional (1D) chain share a corner, thereby forming a twodimensional sheet (2D) in the $a b$ plane (Fig. 1b). Neighbouring sheets are connected to each other by $\mathrm{Fe} 2$ cations, giving rise to a complicated three-dimensional structure (Figs. 2 and 3). It should be noted that the double sodium-iron(II) paratungstate $\mathrm{B} \mathrm{Na} \mathrm{Fe}_{2.5}\left[\mathrm{~W}_{12} \mathrm{O}_{40}(\mathrm{OH})_{2}\right] \cdot 36 \mathrm{H}_{2} \mathrm{O}$ reported in this work has the same cationic composition as reported in $\mathrm{Na}_{5}\left[\left\{\mathrm{Fe}\left(\mathrm{H}_{2} \mathrm{O}\right)_{3}\right\}_{2}\left\{\mathrm{Fe}\left(\mathrm{H}_{2} \mathrm{O}\right)_{4}\right\}_{0.5}\left(\mathrm{H}_{2} \mathrm{~W}_{12} \mathrm{O}_{42}\right)\right] \cdot 30 \mathrm{H}_{2} \mathrm{O}$ (Yang et al., 2003) (Table 1). However, the minor difference with respect to the water content in these two structures leads to a significant change in the unit-cell parameters (Tables 1 and 2) and the motif of crystal packing (Figs. 2 and 3).

In the crystal structure of $\mathbf{N a}_{4} \mathbf{C u}_{3} \mathbf{p a r a B}$, each paratungstate $\mathrm{B}$ anion is coordinated to six $\mathrm{Cu}^{2+}$ and six $\mathrm{Na}^{+}$via $\mathrm{O}_{\mathrm{t}}$ and therefore acts as a dodecadentate ligand (Figs. $1 c$ and $1 d$ ). There are three crystallographically unique copper centres with different coordination modes. Two ( $\mathrm{Cu} 1$ and $\mathrm{Cu} 2)$ out of three $\mathrm{Cu}^{2+}$ cations take part in the formation of infinite chains with alternating $\mathrm{Na}$ and $\mathrm{Cu}$ polyhedra connected by a common edge (Figs. $1 d$ and 4 ) and have different coordination environments. The $\mathrm{Cu} 2$ atoms are linked by four $\mathrm{O}_{\mathrm{t}}$ atoms of the belt-fragment $\left\{\mathrm{W}_{3} \mathrm{O}_{14}\right\}$ from two neighbouring $\left[\mathrm{W}_{12} \mathrm{O}_{40^{-}}\right.$ $\left.(\mathrm{OH})_{2}\right]^{10-}$ anions and two $\mathrm{Na}^{+}$bridging $\mathrm{H}_{2} \mathrm{O}$ molecules. The coordination sphere of $\mathrm{Cu} 3$ consists of two $\mathrm{O}_{\mathrm{t}}$ of the capping $\left\{\mathrm{W}_{3} \mathrm{O}_{13}\right\}$ group of a neighbouring polyanion and is completed by four bridging $\mathrm{H}_{2} \mathrm{O}$ molecules. The third $\mathrm{Cu} 1$ atom coordinates to four $\mathrm{O}_{\mathrm{t}}$ atoms of the belt units $\left\{\mathrm{W}_{3} \mathrm{O}_{14}\right\}$ from two neighbouring $\left[\mathrm{W}_{12} \mathrm{O}_{40}(\mathrm{OH})_{2}\right]^{10-}$ anions and two $\mathrm{H}_{2} \mathrm{O}$ molecules. The $\mathrm{Cu}^{2+}$ ions exhibit a distorted square-bipyramidal coordination geometry with elongated axial distances [2.365 (7)-2.520 (8) $\AA$ ]. The three-dimensional (3D) structure of $\mathbf{N a}_{4} \mathbf{C u}_{3}$-paraB consists of $2 \mathrm{D}$ sheets formed by two chains, namely $\left\{\left[\left(\mathrm{Na}\left(\mathrm{H}_{2} \mathrm{O}\right)_{2}\right)_{2} \mathrm{~W}_{12} \mathrm{O}_{40}(\mathrm{OH})_{2}\right]^{8-}\right\}_{n}$ and $\left\{\left[\mathrm{Na}\left(\mathrm{H}_{2} \mathrm{O}\right)_{2^{-}}\right.\right.$ $\left.\left.\mathrm{Cu}\left(\mathrm{H}_{2} \mathrm{O}\right)_{2}-\mathrm{Na}\left(\mathrm{H}_{2} \mathrm{O}\right)_{2}-\mathrm{Cu}\left(\mathrm{H}_{2} \mathrm{O}\right)_{4}\right]^{6+}\right\}_{n}$ parallel to the $a b$ plane (Fig. 4). The 2D sheets are connected along the $c$ axis by $\left[\mathrm{Cu}\left(\mathrm{H}_{2} \mathrm{O}\right)_{4}\right]^{2+}$ cations (Fig. 5). The two $\left[\mathrm{Na}\left(\mathrm{H}_{2} \mathrm{O}\right)_{5}\right]^{+}$cations, which are connected to $\mathrm{O}_{t}$ of one polyanion and do not participate in the formation of sodium-copper chains, are located in 1D tunnels in the structure of $\mathbf{N a}_{\mathbf{4}} \mathbf{C u}_{\mathbf{3}}$-paraB.

The results of the powder XRD patterns of $\mathbf{N a}_{5} \mathbf{F e}_{2.5}$ paraB and $\mathbf{N a}_{4} \mathbf{C u}_{3}$ paraB have been investigated in the solid state at room temperature (Fig. 6). The simulated powder diffraction pattern was based on the single-crystal structural data. The simulated peak positions are in good agreement with those observed. A comparison of the experimental and simulated powder diffraction patterns confirms that the POTs structures had been solved accurately and that both products consist of a single phase.

In the IR spectra of $\mathbf{N a}_{5} \mathbf{F e}_{2.5}$ paraB and $\mathbf{N a}_{4} \mathbf{C} \mathbf{u}_{3}$ paraB, the characteristic peaks at 975, 950, 932, 867, 676 and $488 \mathrm{~cm}^{-1}$, and at $972,937,926,872,675$ and $493 \mathrm{~cm}^{-1}$, respectively, are attributed to the $\mathrm{W}=\mathrm{O}_{\mathrm{t}}$ and $\mathrm{W}-\mathrm{O}-\mathrm{W}$ vibrations in the

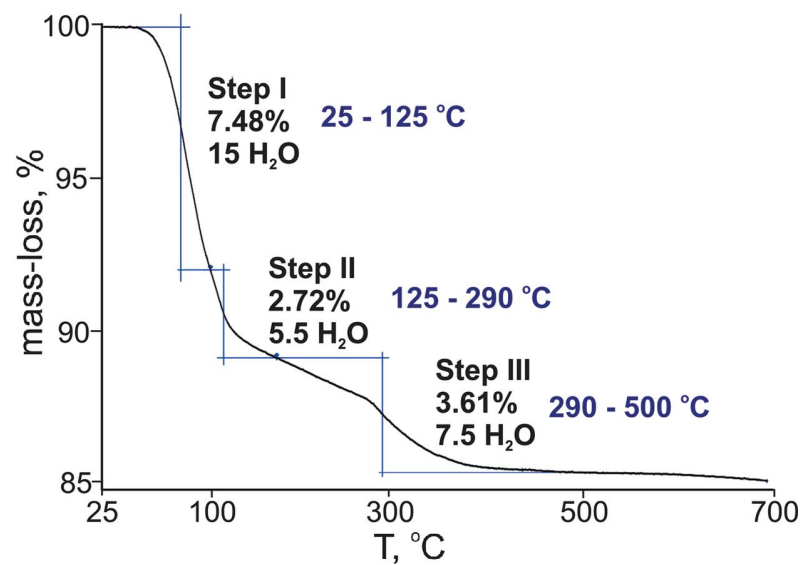

Figure 7

Thermogravimetric curve of $\mathbf{N a}_{\mathbf{4}} \mathrm{Cu}_{3}$ paraB.

paratungstate anion, which are in agreement with previously reported data (Table 1; Qu et al., 2012). The slight peak displacements are due to the effects of different coordination modes of paratungstate $B$. The peaks at $\sim 1600$ and $3400 \mathrm{~cm}^{-1}$ are attributed to the vibration of water molecules.

The disordered water molecules in $\mathbf{N a}_{\mathbf{4}} \mathbf{C} \mathbf{u}_{3}$ paraB were treated with SQUEEZE (Spek, 2015) and the exact number of water molecules was determined by TGA. The TG curve shows a three-step weight-loss process (Fig. 7). The first weight loss of $7.48 \%$ in the temperature range $25-125^{\circ} \mathrm{C}$ corresponds to all lattice $\mathrm{H}_{2} \mathrm{O}$ and water molecules from coordinating $\mathrm{Na}^{+}$ and $\mathrm{Cu}^{2+}$. The second $(2.72 \%)$ and third $(3.61 \%)$ steps in the range $125-500{ }^{\circ} \mathrm{C}$ correspond to $13 \mathrm{H}_{2} \mathrm{O}$ molecules coordinating $\mathrm{Na}^{+}$and $\mathrm{Cu}^{2+}$. The total weight loss is $13.83 \%$, which results in the formula $\mathrm{Na}_{4} \mathrm{Cu}_{3}\left[\mathrm{~W}_{12} \mathrm{O}_{40}(\mathrm{OH})_{2}\right] \cdot 28 \mathrm{H}_{2} \mathrm{O}$.

The success in synthesizing $\mathbf{N a}_{5} \mathbf{F e}_{2.5}$ paraB and $\mathbf{N a}_{\mathbf{4}} \mathbf{C} \mathbf{u}_{\mathbf{3}}$ paraB shows that paratungstate $\mathrm{B}$ is a versatile building block, which can be modified by metal sites into high-dimensional architectures and the different connection principle of the transition metals has a big impact on the dimensionalities of the frameworks.

\section{Acknowledgements}

The authors are grateful to Assistant Professor Dr P. Unfried for support with the TGA and to Associate Professor K. Richter and M. Andraghetti for help with PXRD measurements at the Department of Inorganic Chemistry, University of Vienna. We thank RNDr Marek Bujdoš, PhD, for support with the ICP-MS and AAS analysis at the Institute of Laboratory Research on Geomaterials, Comenius Univiversity in Bratislava. We wish to thank E. Al-Sayed, MSc, and Dr J. Breibeck for valuable discussions regarding this work.

\section{Funding information}

Funding for this research was provided by: Austrian Science Fund (award No. M2203 to N. Gumerova; award No. P27534 to A. Rompel). 


\section{References}

Bijelic, A., Aureliano, M. \& Rompel, A. (2018a). Angew. Chem. Int. $E d$. In the press. doi:10.1002/anie.201803868 and doi:10.1002/ ange. 201803868.

Bijelic, A., Aureliano, M. \& Rompel, A. (2018b). Chem. Commun. 54, 1153-1169.

Bijelic, A. \& Rompel, A. (2015). Coord. Chem. Rev. 299, 22-38.

Bijelic, A. \& Rompel, A. (2017). Acc. Chem. Res. 50, 1441-1448.

Brandenburg, K. (2006). DIAMOND. Crystal Impact GbR, Bonn, Germany.

Brown, I. D. \& Altermatt, D. (1985). Acta Cryst. B41, 244-247.

Bruker (2015). SAINT. Bruker AXS Inc., Madison, Wisconsin, USA.

Bruker (2016). SADABS. Bruker AXS Inc., Madison, Wisconsin, USA.

Chen, Y., Zhang, C., Zhang, J., Ye, Z., Zheng, K., Fang, Q. \& Li, G. (2017). Inorg. Chem. Front. 4, 1917-1922.

Clemente-Juan, J. M., Coronado, E. \& Gaita-Ariño, A. (2012). Chem. Soc. Rev. 41, 7464-7478.

Dolomanov, O. V., Bourhis, L. J., Gildea, R. J., Howard, J. A. K. \& Puschmann, H. (2009). J. Appl. Cryst. 42, 339-341.

Evans, H. T. \& Prince, E. (1983). J. Am. Chem. Soc. 105, 4838-4839.

Evans, H. T. \& Rollins, O. W. (1976). Acta Cryst. B32, 1565-1567.

Fu, L., Gao, H., Yan, M., Li, S., Li, X., Dai, Z. \& Liu, S. (2015). Small, 11, 2938-2945.

Gao, S., Zhao, J., Zhou, B., Yu, K., Su, Z., Wang, L., Yin, Y., Zhao, Z., Yu, Y. \& Chen, Y. (2011). Inorg. Chim. Acta, 379, 151-157.

Groom, C. R., Bruno, I. J., Lightfoot, M. P. \& Ward, S. C. (2016). Acta Cryst. B72, 171-179.

Gumerova, N. I., Kasyanova, K. V., Rozantsev, G. M., Baumer, V. N. \& Radio, S. V. (2015). J. Cluster Sci. 26, 1171-1186.

He, L.-W., Lin, B.-Z., Liu, X.-Z., Huang, X.-F. \& Feng, Y.-L. (2008). Solid State Sci. 10, 237-243.

Hübschle, C. B., Sheldrick, G. M. \& Dittrich, B. (2011). J. Appl. Cryst. 44, 1281-1284.

Kong, Q.-J., Zhang, C.-J. \& Chen, Y.-G. (2010). J. Mol. Struct. 964, 8287.
Li, B., Bi, B., Li, W. \& Wu, L. (2008). J. Solid State Chem. 181, 33373343.

Li, Y.-W., Wang, Y.-H., Li, Y.-G., Wang, E.-B., Chen, W.-L., Wu, Q. \& Shi, Q. (2009). Inorg. Chim. Acta, 362, 1078-1082.

Molitor, C., Bijelic, A. \& Rompel, A. (2017). IUCrJ, 4, 734-740.

Peresypkina, E. V., Virovets, A. V., Adonin, S. A., Abramov, P. A., Rogachev, A. V., Sinkevich, P. L., Korenev, V. S. \& Sokolov, M. N. (2014). J. Struct. Chem. 55, 295-298.

Pope, M. (1983). In Heteropoly and Isopoly Oxometalates. Berlin: Springer.

Proust, A., Thouvenot, R. \& Gouzerh, P. (2008). Chem. Commun. pp. 1837-1852.

Qu, X., Yang, Y., Yu, X., Lv, Z., Ji, M. \& Feng, S. (2015). Inorg. Chem. Commun. 60, 126-130.

Qu, X., Yang, Y., Zhang, F. \& Yu, X. (2012). Struct. Chem. 23, 18671872.

Radio, S. V., Kryuchkov, M. A., Zavialova, E. G., Baumer, V. N., Shishkin, O. V. \& Rozantsev, G. M. (2010). J. Coord. Chem. 63, 1678-1689.

Radio, S. V., Melnik, N. A., Ivantsova, E. S. \& Baumer, V. N. (2014). J. Struct. Chem. 55, 879-886.

Radio, S. V., Rozantsev, G. M., Baumer, V. N. \& Shishkin, O. V. (2011). J. Struct. Chem. 52, 111-117.

Sadakane, M. \& Steckhan, E. (1998). Chem. Rev. 98, 219-238.

Sheldrick, G. M. (2008). Acta Cryst. A64, 112-122.

Sheldrick, G. M. (2015). Acta Cryst. C71, 3-8.

Spek, A. L. (2015). Acta Cryst. C71, 9-18.

Sokolov, M. N., Adonin, S. A., Abramov, P. A., Mainichev, D. A., Zakharchuk, N. F. \& Fedin, V. P. (2012). Chem. Commun. 48, 66666668.

Wang, S.-S. \& Yang, G.-Y. (2015). Chem. Rev. 115, 4893-4962.

Yamase, T. \& Pope, M. (2002). Editors. Polyoxometalate Chemistry for Nano-Composite Design. Springer Science \& Business Media.

Yang, W.-B., Lu, C.-Z., Lin, X. \& Zhuang, H.-H. (2003). Z. Anorg. Allg. Chem. 629, 2046-2052.

Zhang, Z. (2012). J. Chem. Crystallogr. 42, 333-337. 


\section{supporting information}

Acta Cryst. (2018). C74, 1252-1259 [https://doi.org/10.1107/S2053229618010021]

\section{Iron(II) and copper(II) paratungstates B: a single-crystal X-ray diffraction study}

\section{Nadiia I. Gumerova, Anatolie Dobrov, Alexander Roller and Annette Rompel}

\section{Computing details}

For both structures, data collection: APEX3; cell refinement: SAINT (Bruker, 2015); data reduction: SAINT (Bruker, 2015). Program(s) used to solve structure: SHELXS97 (Sheldrick, 2008) and shelXle (Hübschle et al., 2011) for ando209_p-1; SHELXS97 (Sheldrick, 2008) for mo_ando241_p-1. Program(s) used to refine structure: SHELXL2014 (Sheldrick, 2015) for ando209_p-1; SHELXL2016 (Sheldrick, 2015) for mo_ando241_p-1. Molecular graphics: OLEX2 (Dolomanov et al., 2009) and DIAMOND (Brandenburg, 2006) for ando209_p-1; OLEX2 (Dolomanov et al., 2009) for mo_ando241_p-1. For both structures, software used to prepare material for publication: OLEX2 (Dolomanov et al., 2009).

(ando209_p-1)

\section{Crystal data}

$\mathrm{Na}_{4} \mathrm{Cu}_{3}\left[\mathrm{~W}_{12} \mathrm{O}_{40}(\mathrm{OH})_{2}\right] \cdot 28 \mathrm{H}_{2} \mathrm{O}$

$M_{r}=3621.13$

Triclinic, $P \overline{1}$

$a=10.6516(5) \AA$

$b=12.7532(6) \AA$

$c=13.0730(5) \AA$

$\alpha=113.771(1)^{\circ}$

$\beta=90.443(1)^{\circ}$

$\gamma=112.502(1)^{\circ}$

$V=1473.65(11) \AA^{3}$

\section{Data collection}

Bruker APEX-II CCD diffractometer

Radiation source: sealed xray tube, Incoatec IuS $\varphi$ and $\omega$ scans

Absorption correction: multi-scan

(SADABS; Bruker, 2016)

$T_{\min }=0.004, T_{\max }=0.023$

11292 measured reflections

\section{Refinement}

Refinement on $F^{2}$

Least-squares matrix: full

$R\left[F^{2}>2 \sigma\left(F^{2}\right)\right]=0.035$

$w R\left(F^{2}\right)=0.098$

$S=1.05$

5321 reflections

463 parameters

$$
\begin{aligned}
& Z=1 \\
& F(000)=1607 \\
& D_{\mathrm{x}}=4.080 \mathrm{Mg} \mathrm{m}^{-3}
\end{aligned}
$$

Mo $K \alpha$ radiation, $\lambda=0.71073 \AA$

Cell parameters from 6027 reflections

$\theta=3.0-30.0^{\circ}$

$\mu=24.53 \mathrm{~mm}^{-1}$

$T=100 \mathrm{~K}$

Plate, blue

$0.13 \times 0.07 \times 0.02 \mathrm{~mm}$

5321 independent reflections 4720 reflections with $I>2 \sigma(I)$

$R_{\text {int }}=0.048$

$\theta_{\max }=25.4^{\circ}, \theta_{\min }=2.1^{\circ}$

$h=-12 \rightarrow 12$

$k=-15 \rightarrow 14$

$l=-11 \rightarrow 15$

\section{0 restraints}

Hydrogen site location: difference Fourier map

$\mathrm{H}$ atoms treated by a mixture of independent and constrained refinement

$w=1 /\left[\sigma^{2}\left(F_{0}^{2}\right)+(0.0463 P)^{2}+6.2838 P\right]$

where $P=\left(F_{\mathrm{o}}{ }^{2}+2 F_{\mathrm{c}}{ }^{2}\right) / 3$

$(\Delta / \sigma)_{\max }<0.001$ 
$\Delta \rho_{\max }=2.15$ e $\AA^{-3}$

$\Delta \rho_{\min }=-1.63$ e $\AA^{-3}$
Extinction correction: SHELXL2014

(Sheldrick, 2015),

$\mathrm{Fc}^{*}=\mathrm{kFc}\left[1+0.001 \mathrm{xFc}^{2} \lambda^{3} / \sin (2 \theta)\right]^{-1 / 4}$

Extinction coefficient: $0.00022(6)$

Special details

Geometry. All esds (except the esd in the dihedral angle between two 1.s. planes) are estimated using the full covariance matrix. The cell esds are taken into account individually in the estimation of esds in distances, angles and torsion angles; correlations between esds in cell parameters are only used when they are defined by crystal symmetry. An approximate (isotropic) treatment of cell esds is used for estimating esds involving l.s. planes.

Refinement._olex2_refinement_description

1. Fixed Uiso At 1.5 times of: All $\mathrm{O}(\mathrm{H}, \mathrm{H})$ groups 2. Restrained distances Na2-H31A 2.8 with sigma of $0.05 \mathrm{H} 34 \mathrm{~B}-\mathrm{H} 25 \mathrm{~A}$ 2.2 with sigma of 0.02 H34A-H25A 2.2 with sigma of 0.03 H34B_\$2-H31B 2.2 with sigma of 0.02 H34A_\$4-H31B_\$3 2.2 with sigma of $0.02 \mathrm{Cu} 3-\mathrm{H} 25 \mathrm{~A} 2.7$ with sigma of $0.05 \mathrm{Cu} 3-\mathrm{H} 25 \mathrm{~B} 2.3$ with sigma of $0.02 \mathrm{H} 35 \mathrm{~A}-\mathrm{O} 241.8$ with sigma of $0.06 \mathrm{H} 35 \mathrm{~B}-\mathrm{O} 192$ with sigma of $0.06 \mathrm{Na} 1-\mathrm{H} 27 \mathrm{~B} 2.8$ with sigma of $0.05 \mathrm{Na} 1-\mathrm{H} 27 \mathrm{C}=\mathrm{Na} 1-\mathrm{H} 27 \mathrm{~A}=\mathrm{Na} 1-\mathrm{H} 27 \mathrm{D} 2.8$ with sigma of $0.05 \mathrm{H} 33 \mathrm{~B}-\mathrm{Na} 12.8$ with sigma of $0.02 \mathrm{H} 33 \mathrm{~B}-\mathrm{O} 272.4$ with sigma of $0.05 \mathrm{Na} 1-\mathrm{H} 28 \mathrm{~A} 2.7$ with sigma of $0.04 \mathrm{H} 33 \mathrm{~B}-\mathrm{H} 28 \mathrm{~A} 2.4$ with sigma of $0.04 \mathrm{H} 32 \mathrm{~A}$ \$3-O26 1.8 with sigma of $0.04 \mathrm{Na} 2-\mathrm{H} 31 \mathrm{~B}=\mathrm{Na} 2-\mathrm{H} 32 \mathrm{~B} 2.8$ with sigma of 0.075 O32-H27B_\$5 2.3 with sigma of 0.04 H29A_\$1-H22A 2.2 with sigma of 0.02 O31-H31A = O31-H31B = O26$\mathrm{H} 26 \mathrm{~B}=\mathrm{O} 26-\mathrm{H} 26 \mathrm{~A}=\mathrm{O} 29-\mathrm{H} 29 \mathrm{~B}=\mathrm{O} 29-\mathrm{H} 29 \mathrm{~A}=\mathrm{O} 30-\mathrm{H} 30 \mathrm{~A}=\mathrm{O} 30-\mathrm{H} 30 \mathrm{~B}=\mathrm{O} 32-\mathrm{H} 32 \mathrm{~A}=\mathrm{O} 32-\mathrm{H} 32 \mathrm{~B}=\mathrm{O} 27-\mathrm{H} 27 \mathrm{~A}=$ $\mathrm{O} 27-\mathrm{H} 27 \mathrm{~B}=\mathrm{O} 22-\mathrm{H} 22 \mathrm{~A}=\mathrm{O} 22-\mathrm{H} 22 \mathrm{~B}=\mathrm{O} 25-\mathrm{H} 25 \mathrm{~A}=\mathrm{O} 25-\mathrm{H} 25 \mathrm{~B}=\mathrm{O} 28-\mathrm{H} 28 \mathrm{~A}=\mathrm{O} 28-\mathrm{H} 28 \mathrm{~B}=\mathrm{O} 33-\mathrm{H} 33 \mathrm{~A}=\mathrm{O} 33-\mathrm{H} 33 \mathrm{~B}$ 0.87 with sigma of $0.015 \mathrm{Na} 1$ \$5-H29A \$5 2.7 with sigma of $0.04 \mathrm{H} 31 \mathrm{~A}-\mathrm{H} 31 \mathrm{~B} \sim \mathrm{H} 32 \mathrm{~B}-\mathrm{H} 32 \mathrm{~A} \sim \mathrm{H} 29 \mathrm{~A}-\mathrm{H} 29 \mathrm{~B} \sim \mathrm{H} 30 \mathrm{~A}-$ $\mathrm{H} 30 \mathrm{~B} \sim \mathrm{H} 26 \mathrm{~B}-\mathrm{H} 26 \mathrm{~A} \sim \mathrm{H} 27 \mathrm{~A}-\mathrm{H} 27 \mathrm{~B} \sim \mathrm{H} 22 \mathrm{~A}-\mathrm{H} 22 \mathrm{~B} \sim \mathrm{H} 25 \mathrm{~A}-\mathrm{H} 25 \mathrm{~B} \sim \mathrm{H} 28 \mathrm{~A}-\mathrm{H} 28 \mathrm{~B} \sim \mathrm{H} 33 \mathrm{~A}-\mathrm{H} 33 \mathrm{~B}$ with sigma of 0.013. Uiso/Uaniso restraints and constraints Uanis $(\mathrm{O} 3) \sim \mathrm{Ueq}$ : with sigma of 0.01 and sigma for terminal atoms of 0.02 Uanis $(\mathrm{O} 35) \sim$ Ueq: with sigma of 0.01 and sigma for terminal atoms of $0.02 \operatorname{Uanis}(\mathrm{O} 27)=\operatorname{Uanis}(\mathrm{O} 27 \mathrm{~B})=\operatorname{Uanis}(\mathrm{O} 33) 4$. Others Fixed Sof: O27(0.5) H27A(0.5) H27B(0.5) O27B(0.5) H27C(0.5) H27D(0.5) O33(0.2) H33A(0.2) H33B(0.2) O34(0.8) H34A(0.8) H34B(0.8) 5.a Free rotating group: O27B(H27C,H27D), O35(H35A,H35B), O34(H34A,H34B) 5.b Rotating group: $\mathrm{O} 26(\mathrm{H} 26 \mathrm{~A}, \mathrm{H} 26 \mathrm{~B}), \mathrm{O} 30(\mathrm{H} 30 \mathrm{~A}, \mathrm{H} 30 \mathrm{~B})$

smtbx_masks_special_details? loop__smtbx_masks_void_nr_smtbx_masks_void_average_x _smtbx_masks_void_average_y_smtbx_masks_void_average_z_smtbx_masks_void_volume _smtbx_masks_void_count_electrons_smtbx_masks_void_content $10 . \overline{500} 0.0000 .00042 .550 .5$ ?

Fractional atomic coordinates and isotropic or equivalent isotropic displacement parameters $\left(\AA^{2}\right)$

\begin{tabular}{lllll}
\hline & $x$ & $y$ & $z$ & $U_{\text {iso }} * U_{\text {eq }}$ \\
W1 & $0.40078(5)$ & $0.59904(4)$ & $0.70077(4)$ & $0.02587(13)$ \\
W2 & $0.29232(5)$ & $0.29326(4)$ & $0.70980(4)$ & $0.02647(13)$ \\
W3 & $0.11342(5)$ & $0.30259(4)$ & $0.50852(4)$ & $0.02644(13)$ \\
W4 & $0.36784(5)$ & $0.55467(4)$ & $0.25109(4)$ & $0.02598(13)$ \\
W5 & $0.25460(5)$ & $0.24015(4)$ & $0.24532(4)$ & $0.02651(13)$ \\
W6 & $0.44888(5)$ & $0.25037(4)$ & $0.45361(4)$ & $0.02571(13)$ \\
Cu1 & 0.5000 & 0.5000 & 0.0000 & $0.0295(4)$ \\
Cu2 & 0.5000 & 0.0000 & 0.5000 & $0.0318(4)$ \\
Cu3 & 0.0000 & 0.0000 & 0.5000 & $0.0292(4)$ \\
Na1 & $0.1011(5)$ & $0.6455(5)$ & $0.8881(4)$ & $0.0388(11)$ \\
Na2 & $0.2217(6)$ & $-0.0459(5)$ & $0.6480(4)$ & $0.0453(12)$ \\
O1 & $0.4360(8)$ & $0.7354(7)$ & $0.6568(6)$ & $0.0307(17)$ \\
O2 & $0.2961(8)$ & $0.6304(7)$ & $0.7964(6)$ & $0.0321(18)$ \\
O3 & $0.2806(8)$ & $0.4797(7)$ & $0.5682(6)$ & $0.0274(16)$ \\
O4 & $0.4464(8)$ & $0.4809(7)$ & $0.7296(6)$ & $0.0287(17)$ \\
O5 & $0.2953(7)$ & $0.3510(7)$ & $0.8548(6)$ & $0.0278(16)$ \\
O6 & $0.1920(8)$ & $0.1290(7)$ & $0.6530(7)$ & $0.0311(17)$ \\
O7 & $0.4689(7)$ & $0.2862(7)$ & $0.7099(6)$ & $0.0265(16)$
\end{tabular}




\begin{tabular}{|c|c|c|c|c|c|}
\hline O8 & $0.1595(8)$ & $0.3407(7)$ & $0.6693(6)$ & $0.0280(17)$ & \\
\hline O9 & $0.3000(7)$ & $0.2617(7)$ & $0.5253(6)$ & $0.0256(16)$ & \\
\hline $\mathrm{O} 10$ & $0.0060(8)$ & $0.1394(7)$ & $0.4742(6)$ & $0.0283(17)$ & \\
\hline O11 & $0.1443(7)$ & $0.2788(7)$ & $0.3603(6)$ & $0.0273(16)$ & \\
\hline O12 & $-0.0075(8)$ & $0.3650(7)$ & $0.5232(6)$ & $0.0303(17)$ & \\
\hline O13 & $0.3723(8)$ & $0.5048(7)$ & $0.1034(6)$ & $0.0303(17)$ & \\
\hline O14 & $0.2479(8)$ & $0.6201(7)$ & $0.2670(6)$ & $0.0292(17)$ & \\
\hline O15 & $0.2601(8)$ & $0.3979(7)$ & $0.2454(6)$ & $0.0285(17)$ & \\
\hline O16 & $0.4167(8)$ & $0.2715(7)$ & $0.1837(6)$ & $0.0277(17)$ & \\
\hline O17 & $0.1273(8)$ & $0.1263(7)$ & $0.1285(6)$ & $0.0321(18)$ & \\
\hline O18 & $0.4261(8)$ & $0.3860(7)$ & $0.3991(6)$ & $0.0270(16)$ & \\
\hline O19 & $0.2939(8)$ & $0.1433(7)$ & $0.3058(6)$ & $0.0278(16)$ & \\
\hline $\mathrm{O} 20$ & $0.4522(8)$ & $0.1175(7)$ & $0.4616(6)$ & 0.0308 (17) & \\
\hline $\mathrm{O} 21$ & $0.5820(8)$ & $0.3799(7)$ & $0.5722(6)$ & $0.0278(17)$ & \\
\hline $\mathrm{O} 22$ & $0.5004(8)$ & $0.6562(7)$ & $-0.0030(7)$ & $0.0322(18)$ & \\
\hline $\mathrm{H} 22 \mathrm{~A}$ & $0.484(6)$ & $0.641(10)$ & $-0.074(3)$ & $0.048 *$ & \\
\hline $\mathrm{H} 22 \mathrm{~B}$ & $0.572(9)$ & $0.722(8)$ & $0.045(6)$ & $0.048^{*}$ & \\
\hline $\mathrm{O} 23$ & $0.2659(9)$ & $-0.1426(8)$ & $0.4500(7)$ & $0.039(2)$ & \\
\hline $\mathrm{O} 24$ & $0.5333(8)$ & $-0.0689(7)$ & $0.3444(7)$ & $0.0329(18)$ & \\
\hline $\mathrm{O} 25$ & $0.1090(9)$ & $-0.0398(7)$ & $0.3744(7)$ & $0.039(2)$ & \\
\hline $\mathrm{H} 25 \mathrm{~A}$ & $0.110(4)$ & $-0.105(2)$ & 0.3192 (19) & $0.059^{*}$ & \\
\hline $\mathrm{H} 25 \mathrm{~B}$ & $0.166(10)$ & $0.037(2)$ & $0.392(8)$ & $0.059^{*}$ & \\
\hline $\mathrm{O} 26$ & $0.0069(9)$ & $0.5944(8)$ & $0.7000(7)$ & $0.0376(19)$ & \\
\hline $\mathrm{H} 26 \mathrm{~A}$ & -0.0758 & 0.6059 & 0.6943 & $0.056^{*}$ & \\
\hline $\mathrm{H} 26 \mathrm{~B}$ & -0.0175 & 0.5090 & 0.6477 & $0.056^{*}$ & \\
\hline $\mathrm{O} 28$ & $-0.0711(10)$ & $0.6370(10)$ & $1.0034(8)$ & $0.053(3)$ & \\
\hline $\mathrm{H} 28 \mathrm{~A}$ & $-0.148(3)$ & $0.585(9)$ & $0.954(3)$ & $0.080^{*}$ & \\
\hline $\mathrm{H} 28 \mathrm{~B}$ & $-0.056(11)$ & 0.635 (12) & $1.068(7)$ & $0.080 *$ & \\
\hline O29 & $0.2535(9)$ & $0.6809(10)$ & $1.0510(8)$ & $0.047(2)$ & \\
\hline $\mathrm{H} 29 \mathrm{~A}$ & $0.310(7)$ & $0.654(10)$ & $1.013(4)$ & $0.070^{*}$ & \\
\hline H29B & $0.282(10)$ & $0.757(6)$ & $1.107(9)$ & $0.070 *$ & \\
\hline $\mathrm{O} 30$ & $0.0122(9)$ & $0.4225(8)$ & $0.8273(7)$ & $0.039(2)$ & \\
\hline H30A & 0.0498 & 0.3733 & 0.7612 & $0.058 *$ & \\
\hline H30B & -0.0916 & 0.3696 & 0.7900 & $0.058^{*}$ & \\
\hline O31 & $0.2749(14)$ & 0.1008 (11) & $0.8572(10)$ & $0.072(3)$ & \\
\hline H31A & $0.306(13)$ & $0.060(8)$ & $0.883(4)$ & $0.107^{*}$ & \\
\hline H31B & $0.196(6)$ & $0.105(11)$ & $0.867(7)$ & $0.107^{*}$ & \\
\hline $\mathrm{O} 32$ & $0.2210(10)$ & $-0.1857(9)$ & $0.7127(9)$ & $0.051(2)$ & \\
\hline $\mathrm{H} 32 \mathrm{~A}$ & $0.150(6)$ & $-0.252(9)$ & $0.712(13)$ & $0.077^{*}$ & \\
\hline H32B & $0.305(4)$ & $-0.174(11)$ & $0.736(14)$ & $0.077^{*}$ & \\
\hline $\mathrm{O} 27$ & $0.158(3)$ & $0.860(3)$ & $0.922(2)$ & $0.057(5)$ & 0.5 \\
\hline $\mathrm{H} 27 \mathrm{~A}$ & $0.246(5)$ & $0.904(6)$ & $0.946(10)$ & $0.086^{*}$ & 0.5 \\
\hline H27B & $0.113(6)$ & $0.858(6)$ & $0.865(4)$ & $0.086^{*}$ & 0.5 \\
\hline $\mathrm{O} 27 \mathrm{~B}$ & $0.119(3)$ & $0.853(3)$ & $0.960(2)$ & $0.057(5)$ & 0.5 \\
\hline $\mathrm{H} 27 \mathrm{C}$ & 0.0694 & 0.8626 & 0.9158 & $0.086^{*}$ & 0.5 \\
\hline $\mathrm{H} 27 \mathrm{D}$ & 0.2008 & 0.9067 & 0.9675 & $0.086^{*}$ & 0.5 \\
\hline $\mathrm{O} 35$ & $0.3779(17)$ & $-0.0594(14)$ & $0.1911(10)$ & $0.097(5)$ & \\
\hline $\mathrm{H} 35 \mathrm{~A}$ & 0.4125 & -0.0615 & 0.2488 & $0.145^{*}$ & \\
\hline
\end{tabular}




\begin{tabular}{llllll} 
H35B & 0.3375 & -0.0105 & 0.2126 & $0.145^{*}$ & \\
O33 & $0.004(6)$ & $0.884(4)$ & $1.035(4)$ & $0.057(5)$ & 0.2 \\
H33A & $0.07(5)$ & $0.951(12)$ & $1.09(4)$ & $0.086^{*}$ & 0.2 \\
H33B & $-0.012(17)$ & $0.808(8)$ & $1.027(16)$ & $0.086^{*}$ & 0.2 \\
O34 & $-0.0162(12)$ & $-0.1184(11)$ & $0.1541(9)$ & $0.050(3)$ & 0.8 \\
H34A & 0.0167 & -0.0472 & 0.2126 & $0.075^{*}$ & 0.8 \\
H34B & -0.0554 & -0.1769 & 0.1746 & $0.075^{*}$ & 0.8 \\
\hline
\end{tabular}

Atomic displacement parameters $\left(\AA^{2}\right)$

\begin{tabular}{|c|c|c|c|c|c|c|}
\hline & $U^{11}$ & $U^{22}$ & $U^{\beta 3}$ & $U^{12}$ & $U^{13}$ & $U^{23}$ \\
\hline W1 & $0.0266(2)$ & $0.0247(2)$ & $0.0263(2)$ & $0.01145(19)$ & $0.00481(18)$ & 0.01057 (19) \\
\hline W2 & $0.0264(2)$ & $0.0259(2)$ & $0.0273(2)$ & $0.01121(19)$ & $0.00467(18)$ & 0.01163 (19) \\
\hline W3 & $0.0264(2)$ & $0.0253(2)$ & $0.0273(2)$ & $0.01089(19)$ & $0.00448(18)$ & 0.01138 (19) \\
\hline W4 & $0.0262(2)$ & 0.0257 (2) & $0.0260(2)$ & $0.01136(19)$ & $0.00429(18)$ & 0.01098 (19) \\
\hline W5 & $0.0270(2)$ & $0.0250(2)$ & $0.0265(2)$ & 0.01085 (19) & $0.00361(18)$ & 0.01049 (19) \\
\hline W6 & $0.0266(2)$ & 0.0245 (2) & 0.0259 (2) & 0.01144 (19) & $0.00433(18)$ & 0.01039 (19) \\
\hline $\mathrm{Cu} 1$ & $0.0326(10)$ & $0.0310(10)$ & $0.0286(10)$ & $0.0162(9)$ & $0.0076(8)$ & $0.0138(8)$ \\
\hline $\mathrm{Cu} 2$ & $0.0351(11)$ & $0.0298(10)$ & $0.0326(10)$ & $0.0152(9)$ & $0.0068(8)$ & $0.0142(9)$ \\
\hline $\mathrm{Cu} 3$ & $0.0302(10)$ & $0.0274(10)$ & $0.0319(10)$ & $0.0130(8)$ & $0.0059(8)$ & $0.0140(8)$ \\
\hline $\mathrm{Na} 1$ & 0.037 (3) & $0.042(3)$ & $0.039(3)$ & 0.019 (2) & $0.008(2)$ & $0.017(2)$ \\
\hline $\mathrm{Na} 2$ & $0.045(3)$ & $0.037(3)$ & $0.052(3)$ & $0.015(2)$ & $0.004(2)$ & $0.021(2)$ \\
\hline $\mathrm{O} 1$ & $0.027(4)$ & $0.029(4)$ & $0.031(4)$ & 0.009 (3) & $0.002(3)$ & $0.011(3)$ \\
\hline $\mathrm{O} 2$ & $0.032(4)$ & $0.038(4)$ & $0.032(4)$ & 0.017 (4) & $0.004(3)$ & $0.019(4)$ \\
\hline $\mathrm{O} 3$ & $0.028(4)$ & $0.028(4)$ & $0.028(4)$ & 0.009 (3) & 0.009 (3) & $0.016(3)$ \\
\hline $\mathrm{O} 4$ & $0.031(4)$ & $0.025(4)$ & $0.035(4)$ & 0.015 & 0.009 (3) & $0.014(3)$ \\
\hline O5 & $0.023(4)$ & $0.030(4)$ & $0.030(4)$ & $0.010(3)$ & $0.004(3)$ & $0.014(3)$ \\
\hline O6 & $0.031(4)$ & $0.029(4)$ & $0.036(4)$ & 0.015 & 0.005 & 0.015 \\
\hline O7 & $0.024(4)$ & $0.028(4)$ & $0.029(4)$ & $0.012(3)$ & 0.002 & $0.012(3)$ \\
\hline $\mathrm{O} 8$ & $0.026(4)$ & $0.033(4)$ & $0.026(4)$ & 0.011 & 0.005 & 0.015 \\
\hline O9 & $0.026(4)$ & $0.028(4)$ & $0.021(4)$ & 0.009 (3) & $0.004(3)$ & 0.012 \\
\hline $\mathrm{O} 10$ & $0.028(4)$ & $0.023(4)$ & $0.030(4)$ & 0.007 (3) & 0.002 & 0.011 \\
\hline O11 & $0.023(4)$ & $0.031(4)$ & $0.031(4)$ & $0.013(3)$ & $0.004(3)$ & $0.016(3)$ \\
\hline $\mathrm{O} 12$ & $0.032(4)$ & 0.037 (4) & 0.029 (4) & 0.017 (4) & $0.011(3)$ & $0.018(4)$ \\
\hline $\mathrm{O} 13$ & $0.028(4)$ & $0.035(4)$ & $0.029(4)$ & $0.013(3)$ & 0.007 (3) & $0.014(3)$ \\
\hline $\mathrm{O} 14$ & $0.031(4)$ & $0.026(4)$ & $0.030(4)$ & 0.011 & 0.007 (3) & $0.013(3)$ \\
\hline $\mathrm{O} 15$ & $0.029(4)$ & $0.025(4)$ & $0.030(4)$ & 0.009 (3) & $0.006(3)$ & $0.013(3)$ \\
\hline O16 & $0.034(4)$ & $0.030(4)$ & $0.020(4)$ & 0.015 & $0.006(3)$ & 0.010 \\
\hline O17 & $0.039(5)$ & $0.032(4)$ & $0.028(4)$ & $0.017(4)$ & $0.006(3)$ & $0.014(3)$ \\
\hline $\mathrm{O} 18$ & $0.028(4)$ & $0.021(4)$ & $0.029(4)$ & 0.008 & $0.002(3)$ & 0.010 \\
\hline O19 & $0.025(4)$ & $0.028(4)$ & $0.032(4)$ & $0.012(3)$ & 0.007 (3) & 0.013 \\
\hline $\mathrm{O} 20$ & $0.033(4)$ & $0.033(4)$ & $0.030(4)$ & $0.016(4)$ & 0.010 & 0.015 \\
\hline $\mathrm{O} 21$ & $0.024(4)$ & $0.030(4)$ & $0.025(4)$ & 0.008 & $0.006(3)$ & $0.011(3)$ \\
\hline $\mathrm{O} 22$ & $0.033(5)$ & $0.032(4)$ & $0.029(4)$ & $0.017(4)$ & 0.002 & $0.008(4)$ \\
\hline $\mathrm{O} 23$ & $0.037(5)$ & $0.034(4)$ & $0.040(5)$ & $0.016(4)$ & 0.002 (4) & 0.009 (4) \\
\hline $\mathrm{O} 24$ & $0.032(4)$ & $0.029(4)$ & $0.034(4)$ & $0.013(4)$ & 0.003 & $0.010(3)$ \\
\hline $\mathrm{O} 25$ & $0.043(5)$ & $0.024(4)$ & $0.042(5)$ & $0.010(4)$ & $0.012(4)$ & $0.011(4)$ \\
\hline $\mathrm{O} 26$ & $0.036(5)$ & $0.042(5)$ & $0.038(5)$ & $0.020(4)$ & 0.007 (4) & $0.017(4)$ \\
\hline
\end{tabular}




\begin{tabular}{lllllll} 
O28 & $0.043(6)$ & $0.082(7)$ & $0.036(5)$ & $0.032(5)$ & $0.011(4)$ & $0.023(5)$ \\
O29 & $0.044(5)$ & $0.062(6)$ & $0.045(5)$ & $0.031(5)$ & $0.016(4)$ & $0.026(5)$ \\
O30 & $0.036(5)$ & $0.051(5)$ & $0.029(4)$ & $0.021(4)$ & $0.009(4)$ & $0.014(4)$ \\
O31 & $0.102(10)$ & $0.065(7)$ & $0.054(6)$ & $0.043(7)$ & $0.005(6)$ & $0.025(6)$ \\
O32 & $0.039(5)$ & $0.052(6)$ & $0.070(7)$ & $0.021(5)$ & $0.012(5)$ & $0.033(5)$ \\
O27 & $0.070(15)$ & $0.047(7)$ & $0.062(16)$ & $0.028(10)$ & $0.021(9)$ & $0.027(10)$ \\
O27B & $0.070(15)$ & $0.047(7)$ & $0.062(16)$ & $0.028(10)$ & $0.021(9)$ & $0.027(10)$ \\
O35 & $0.126(11)$ & $0.088(9)$ & $0.065(7)$ & $0.078(8)$ & $-0.027(7)$ & $-0.007(6)$ \\
O33 & $0.070(15)$ & $0.047(7)$ & $0.062(16)$ & $0.028(10)$ & $0.021(9)$ & $0.027(10)$ \\
O34 & $0.047(7)$ & $0.041(6)$ & $0.030(6)$ & $-0.005(5)$ & $0.010(5)$ & $0.008(5)$ \\
\hline
\end{tabular}

Geometric parameters $\left(\AA,{ }^{\circ}\right)$

\begin{tabular}{|c|c|c|c|}
\hline $\mathrm{W} 1-\mathrm{O} 1$ & $1.951(7)$ & $\mathrm{Cu} 1-\mathrm{O} 22$ & $2.007(8)$ \\
\hline $\mathrm{W} 1-\mathrm{O} 2$ & $1.710(8)$ & $\mathrm{Cu} 1-\mathrm{O} 22^{\mathrm{iv}}$ & $2.007(8)$ \\
\hline $\mathrm{W} 1-\mathrm{O} 3$ & $1.818(7)$ & $\mathrm{Cu} 2-\mathrm{Na} 2$ & $3.542(6)$ \\
\hline $\mathrm{W} 1-\mathrm{O} 4$ & $1.914(7)$ & $\mathrm{Cu} 2-\mathrm{Na} 2^{\mathrm{v}}$ & $3.542(6)$ \\
\hline $\mathrm{W} 1-\mathrm{O} 16^{\mathrm{i}}$ & $2.067(8)$ & $\mathrm{Cu} 2-\mathrm{O} 20$ & $1.985(8)$ \\
\hline $\mathrm{W} 1-\mathrm{O} 18^{\mathrm{i}}$ & $2.260(8)$ & $\mathrm{Cu} 2-\mathrm{O} 20^{v}$ & $1.985(8)$ \\
\hline $\mathrm{W} 2-\mathrm{O} 4$ & $2.230(7)$ & $\mathrm{Cu} 2-\mathrm{O} 23$ & $2.345(8)$ \\
\hline $\mathrm{W} 2-\mathrm{O} 5$ & $1.730(7)$ & $\mathrm{Cu} 2-\mathrm{O} 23^{\mathrm{v}}$ & $2.345(8)$ \\
\hline $\mathrm{W} 2-\mathrm{O} 6$ & $1.762(8)$ & $\mathrm{Cu} 2-\mathrm{O} 24$ & $1.961(8)$ \\
\hline $\mathrm{W} 2-\mathrm{O} 7$ & $1.917(7)$ & $\mathrm{Cu} 2-\mathrm{O} 24^{\mathrm{v}}$ & $1.961(8)$ \\
\hline $\mathrm{W} 2-\mathrm{O} 8$ & $1.884(8)$ & $\mathrm{Cu} 3-\mathrm{Na} 2$ & $3.400(5)$ \\
\hline $\mathrm{W} 2-\mathrm{O} 9$ & $2.287(7)$ & $\mathrm{Cu} 3-\mathrm{Na} 2^{\mathrm{ii}}$ & $3.400(5)$ \\
\hline $\mathrm{W} 3-\mathrm{Na} 2^{\mathrm{ii}}$ & $3.632(5)$ & $\mathrm{Cu} 3-\mathrm{O} 6$ & $2.366(8)$ \\
\hline $\mathrm{W} 3-\mathrm{O} 3$ & $2.088(7)$ & $\mathrm{Cu} 3-\mathrm{O}^{\mathrm{ii}}$ & $2.366(8)$ \\
\hline $\mathrm{W} 3-\mathrm{O} 8$ & $1.968(7)$ & $\mathrm{Cu} 3-\mathrm{O} 10$ & $1.918(7)$ \\
\hline W3-O9 & $2.268(8)$ & $\mathrm{Cu} 3-\mathrm{O} 10^{\mathrm{ii}}$ & $1.918(7)$ \\
\hline $\mathrm{W} 3-\mathrm{O} 10$ & $1.805(7)$ & $\mathrm{Cu} 3-\mathrm{O} 25^{\mathrm{ii}}$ & $2.027(8)$ \\
\hline $\mathrm{W} 3-\mathrm{O} 11$ & $1.888(7)$ & $\mathrm{Cu} 3-\mathrm{O} 25$ & $2.027(8)$ \\
\hline $\mathrm{W} 3-\mathrm{O} 12$ & $1.730(8)$ & $\mathrm{Na} 1-\mathrm{O} 2$ & $2.439(9)$ \\
\hline $\mathrm{W} 4-\mathrm{O} 4^{\mathrm{i}}$ & $2.226(8)$ & $\mathrm{Na} 1-\mathrm{O} 26$ & $2.376(9)$ \\
\hline $\mathrm{W} 4-\mathrm{O}^{\mathrm{i}}$ & $1.963(7)$ & $\mathrm{Na} 1-\mathrm{O} 28$ & $2.380(10)$ \\
\hline $\mathrm{W} 4-\mathrm{O} 13$ & $1.780(7)$ & $\mathrm{Na} 1-\mathrm{O} 29$ & $2.446(11)$ \\
\hline $\mathrm{W} 4-\mathrm{O} 14$ & $1.747(8)$ & $\mathrm{Na} 1-\mathrm{O} 30$ & $2.392(10)$ \\
\hline $\mathrm{W} 4-\mathrm{O} 15$ & $1.855(7)$ & $\mathrm{Na} 1-\mathrm{O} 27$ & $2.41(3)$ \\
\hline $\mathrm{W} 4-\mathrm{O} 21^{\mathrm{i}}$ & $2.098(7)$ & $\mathrm{Na} 1-\mathrm{O} 27 \mathrm{~B}$ & $2.35(3)$ \\
\hline W5-O11 & $1.938(7)$ & $\mathrm{Na} 2-\mathrm{W} 3^{\mathrm{ii}}$ & $3.632(5)$ \\
\hline $\mathrm{W} 5-\mathrm{O} 15$ & $1.990(7)$ & $\mathrm{Na} 2-\mathrm{O} 6$ & $2.345(9)$ \\
\hline $\mathrm{W} 5-\mathrm{O} 16$ & $1.882(8)$ & $\mathrm{Na} 2-\mathrm{O} 10^{\mathrm{ii}}$ & $2.459(9)$ \\
\hline $\mathrm{W} 5-\mathrm{O} 17$ & $1.718(8)$ & $\mathrm{Na} 2-\mathrm{O} 23$ & $2.516(10)$ \\
\hline $\mathrm{W} 5-\mathrm{O} 18$ & $2.273(7)$ & $\mathrm{Na} 2-\mathrm{O} 24^{\mathrm{v}}$ & $2.432(10)$ \\
\hline $\mathrm{W} 5-\mathrm{O} 19$ & $1.872(7)$ & $\mathrm{Na} 2-\mathrm{O} 31$ & $2.519(13)$ \\
\hline $\mathrm{W} 6-\mathrm{O} 1^{\mathrm{i}}$ & $1.920(8)$ & $\mathrm{Na} 2-\mathrm{O} 32$ & $2.260(10)$ \\
\hline W6-O9 & $1.872(7)$ & $\mathrm{O} 1-\mathrm{W} 6^{\mathrm{i}}$ & $1.920(8)$ \\
\hline $\mathrm{W} 6-\mathrm{O} 18$ & $2.207(7)$ & $\mathrm{O} 4-\mathrm{W} 4^{\mathrm{i}}$ & $2.226(8)$ \\
\hline W6-O19 & $2.103(7)$ & $\mathrm{O} 5-\mathrm{Cu} 1^{\mathrm{vi}}$ & $2.413(7)$ \\
\hline
\end{tabular}




\begin{tabular}{|c|c|}
\hline $\mathrm{W} 6-\mathrm{O} 20$ & $1.753(8)$ \\
\hline $\mathrm{W} 6-\mathrm{O} 21$ & $1.803(7)$ \\
\hline $\mathrm{Cu} 1-\mathrm{O} 5^{\mathrm{iii}}$ & $2.413(7)$ \\
\hline $\mathrm{Cu} 1-\mathrm{O}^{\mathrm{i}}$ & $2.413(7)$ \\
\hline $\mathrm{Cu} 1-\mathrm{O} 13$ & $1.926(8)$ \\
\hline $\mathrm{Cu} 1-\mathrm{O} 13^{\mathrm{iv}}$ & $1.926(8)$ \\
\hline $\mathrm{O} 1-\mathrm{W} 1-\mathrm{O} 16^{\mathrm{i}}$ & $82.4(3)$ \\
\hline $\mathrm{O} 1-\mathrm{W} 1-\mathrm{O} 18^{\mathrm{i}}$ & $70.2(3)$ \\
\hline $\mathrm{O} 2-\mathrm{W} 1-\mathrm{O} 1$ & $99.5(3)$ \\
\hline $\mathrm{O} 2-\mathrm{W} 1-\mathrm{O} 3$ & $104.1(4)$ \\
\hline $\mathrm{O} 2-\mathrm{W} 1-\mathrm{O} 4$ & $102.2(3)$ \\
\hline $\mathrm{O} 2-\mathrm{W} 1-\mathrm{O} 16^{\mathrm{i}}$ & $94.8(3)$ \\
\hline $\mathrm{O} 2-\mathrm{W} 1-\mathrm{O} 18^{\mathrm{i}}$ & $164.6(3)$ \\
\hline $\mathrm{O} 3-\mathrm{W} 1-\mathrm{O} 1$ & $93.2(3)$ \\
\hline $\mathrm{O} 3-\mathrm{W} 1-\mathrm{O} 4$ & $94.1(3)$ \\
\hline $\mathrm{O} 3-\mathrm{W} 1-\mathrm{O} 16^{\mathrm{i}}$ & $161.1(3)$ \\
\hline $\mathrm{O} 3-\mathrm{W} 1-\mathrm{O} 18^{\mathrm{i}}$ & $88.3(3)$ \\
\hline $\mathrm{O} 4-\mathrm{W} 1-\mathrm{O} 1$ & $154.6(3)$ \\
\hline $\mathrm{O} 4-\mathrm{W} 1-\mathrm{O} 16^{\mathrm{i}}$ & $82.8(3)$ \\
\hline $\mathrm{O} 4-\mathrm{W} 1-\mathrm{O} 18^{\mathrm{i}}$ & $85.7(3)$ \\
\hline $\mathrm{O} 16^{\mathrm{i}}-\mathrm{W} 1-\mathrm{O} 18^{\mathrm{i}}$ & $72.9(3)$ \\
\hline O4-W2-O9 & $77.3(3)$ \\
\hline $\mathrm{O} 5-\mathrm{W} 2-\mathrm{O} 4$ & $91.7(3)$ \\
\hline $\mathrm{O} 5-\mathrm{W} 2-\mathrm{O} 6$ & $105.6(3)$ \\
\hline $\mathrm{O} 5-\mathrm{W} 2-\mathrm{O} 7$ & $98.5(3)$ \\
\hline $\mathrm{O} 5-\mathrm{W} 2-\mathrm{O} 8$ & $98.8(3)$ \\
\hline $\mathrm{O} 5-\mathrm{W} 2-\mathrm{O} 9$ & $167.4(3)$ \\
\hline $\mathrm{O} 6-\mathrm{W} 2-\mathrm{O} 4$ & $161.2(3)$ \\
\hline $\mathrm{O} 6-\mathrm{W} 2-\mathrm{O} 7$ & $96.2(3)$ \\
\hline $\mathrm{O} 6-\mathrm{W} 2-\mathrm{O} 8$ & $99.2(3)$ \\
\hline $\mathrm{O} 6-\mathrm{W} 2-\mathrm{O} 9$ & $86.2(3)$ \\
\hline $\mathrm{O} 7-\mathrm{W} 2-\mathrm{O} 4$ & $73.6(3)$ \\
\hline $\mathrm{O} 7-\mathrm{W} 2-\mathrm{O} 9$ & $84.4(3)$ \\
\hline $\mathrm{O} 8-\mathrm{W} 2-\mathrm{O} 4$ & $85.1(3)$ \\
\hline $\mathrm{O} 8-\mathrm{W} 2-\mathrm{O} 7$ & $152.8(3)$ \\
\hline $\mathrm{O} 8-\mathrm{W} 2-\mathrm{O} 9$ & $74.4(3)$ \\
\hline $\mathrm{O} 3-\mathrm{W} 3-\mathrm{Na} 2^{\mathrm{ii}}$ & $159.2(2)$ \\
\hline $\mathrm{O} 3-\mathrm{W} 3-\mathrm{O} 9$ & $76.3(3)$ \\
\hline $\mathrm{O} 8-\mathrm{W} 3-\mathrm{Na} 2^{\mathrm{ii}}$ & $115.9(2)$ \\
\hline $\mathrm{O} 8-\mathrm{W} 3-\mathrm{O} 3$ & $79.4(3)$ \\
\hline $\mathrm{O} 8-\mathrm{W} 3-\mathrm{O} 9$ & $73.4(3)$ \\
\hline $\mathrm{O} 9-\mathrm{W} 3-\mathrm{Na} 2^{\mathrm{ii}}$ & $120.3(2)$ \\
\hline $\mathrm{O} 10-\mathrm{W} 3-\mathrm{Na} 2^{\mathrm{ii}}$ & $37.5(3)$ \\
\hline $\mathrm{O} 10-\mathrm{W} 3-\mathrm{O} 3$ & $163.0(3)$ \\
\hline $\mathrm{O} 10-\mathrm{W} 3-\mathrm{O} 8$ & $90.7(3)$ \\
\hline $\mathrm{O} 10-\mathrm{W} 3-\mathrm{O} 9$ & 87.7 (3) \\
\hline $\mathrm{O} 10-\mathrm{W} 3-\mathrm{O} 11$ & $97.4(3)$ \\
\hline
\end{tabular}

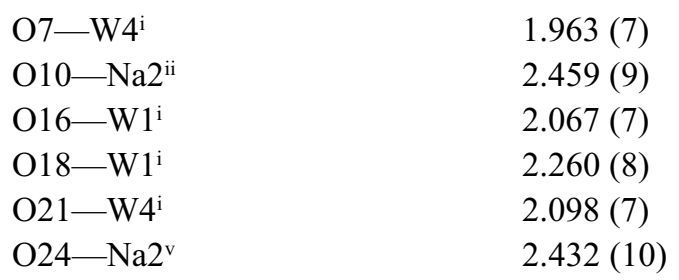

$1.963(7)$

$2.459(9)$

2.067 (7)

$2.260(8)$

$2.098(7)$

$2.432(10)$

$41.1(2)$

138.9 (2)

41.1 (2)

87.1 (3)

$92.9(3)$

87.1 (3)

$92.9(3)$

93.9 (3)

93.9 (3)

86.1 (3)

86.1 (3)

180.0

180.0

$43.6(2)$

$43.6(2)$

136.4 (2)

$136.4(2)$

$180.0(3)$

134.8 (2)

$45.2(2)$

$134.8(2)$

45.2 (2)

86.7 (3)

93.3 (3)

93.3 (3)

86.7 (3)

180.0

89.1 (3)

90.9 (3)

90.9 (3)

89.1 (3)

85.0 (3)

85.0 (3)

95.0 (3)

95.0 (3)

96.9 (3)

83.1 (3)

96.9 (3)

83.1 (3)

180.0 (4)

81.7 (3) 


\begin{tabular}{|c|c|c|c|}
\hline $\mathrm{O} 11-\mathrm{W} 3-\mathrm{Na}^{2 \mathrm{ii}}$ & $82.2(2)$ & $\mathrm{O} 26-\mathrm{Na} 1-\mathrm{O} 2$ & $80.5(3)$ \\
\hline $\mathrm{O} 11-\mathrm{W} 3-\mathrm{O} 3$ & $87.2(3)$ & $\mathrm{O} 26-\mathrm{Na} 1-\mathrm{O} 28$ & $111.0(4)$ \\
\hline $\mathrm{O} 11-\mathrm{W} 3-\mathrm{O} 8$ & $157.1(3)$ & $\mathrm{O} 26-\mathrm{Na} 1-\mathrm{O} 29$ & $161.9(4)$ \\
\hline $\mathrm{O} 11-\mathrm{W} 3-\mathrm{O} 9$ & $85.5(3)$ & $\mathrm{O} 26-\mathrm{Na} 1-\mathrm{O} 30$ & $86.2(3)$ \\
\hline $\mathrm{O} 12-\mathrm{W} 3-\mathrm{Na} 2^{\mathrm{ii}}$ & $71.8(3)$ & $\mathrm{O} 26-\mathrm{Na} 1-\mathrm{O} 27$ & $84.4(8)$ \\
\hline $\mathrm{O} 12-\mathrm{W} 3-\mathrm{O} 3$ & $93.1(3)$ & $\mathrm{O} 28-\mathrm{Na} 1-\mathrm{O} 2$ & $165.5(4)$ \\
\hline $\mathrm{O} 12-\mathrm{W} 3-\mathrm{O} 8$ & $97.5(3)$ & $\mathrm{O} 28-\mathrm{Na} 1-\mathrm{O} 29$ & $86.1(3)$ \\
\hline $\mathrm{O} 12-\mathrm{W} 3-\mathrm{O} 9$ & $167.0(3)$ & $\mathrm{O} 28-\mathrm{Na} 1-\mathrm{O} 30$ & $84.9(4)$ \\
\hline $\mathrm{O} 12-\mathrm{W} 3-\mathrm{O} 10$ & $101.9(4)$ & $\mathrm{O} 28-\mathrm{Na} 1-\mathrm{O} 27$ & $94.3(6)$ \\
\hline $\mathrm{O} 12-\mathrm{W} 3-\mathrm{O} 11$ & $101.7(3)$ & $\mathrm{O} 30-\mathrm{Na} 1-\mathrm{O} 2$ & $87.1(3)$ \\
\hline 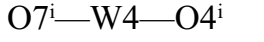 & $72.8(3)$ & $\mathrm{O} 30-\mathrm{Na} 1-\mathrm{O} 29$ & $89.7(4)$ \\
\hline 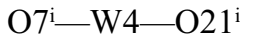 & $79.7(3)$ & $\mathrm{O} 30-\mathrm{Na} 1-\mathrm{O} 27$ & $169.6(9)$ \\
\hline $\mathrm{O} 13-\mathrm{W} 4-\mathrm{O}^{\mathrm{i}}$ & $88.2(3)$ & $\mathrm{O} 27-\mathrm{Na} 1-\mathrm{O} 2$ & $95.8(7)$ \\
\hline $\mathrm{O} 13-\mathrm{W} 4-\mathrm{O} 7^{\mathrm{i}}$ & $91.2(3)$ & $\mathrm{O} 27-\mathrm{Na} 1-\mathrm{O} 29$ & $100.6(9)$ \\
\hline $\mathrm{O} 13-\mathrm{W} 4-\mathrm{O} 15$ & $98.3(3)$ & $\mathrm{O} 27 \mathrm{~B}-\mathrm{Na} 1-\mathrm{O} 2$ & $110.2(7)$ \\
\hline $\mathrm{O} 13-\mathrm{W} 4-\mathrm{O} 21^{\mathrm{i}}$ & $164.6(3)$ & $\mathrm{O} 27 \mathrm{~B}-\mathrm{Na} 1-\mathrm{O} 26$ & $92.8(8)$ \\
\hline $\mathrm{O} 14-\mathrm{W} 4-\mathrm{O}_{4}^{\mathrm{i}}$ & $164.9(3)$ & $\mathrm{O} 27 \mathrm{~B}-\mathrm{Na} 1-\mathrm{O} 28$ & $78.9(6)$ \\
\hline $\mathrm{O} 14-\mathrm{W} 4-\mathrm{O} 7^{\mathrm{i}}$ & $95.4(3)$ & $\mathrm{O} 27 \mathrm{~B}-\mathrm{Na} 1-\mathrm{O} 29$ & $96.3(9)$ \\
\hline $\mathrm{O} 14-\mathrm{W} 4-\mathrm{O} 13$ & $101.8(3)$ & $\mathrm{O} 27 \mathrm{~B}-\mathrm{Na} 1-\mathrm{O} 30$ & $162.2(8)$ \\
\hline $\mathrm{O} 14-\mathrm{W} 4-\mathrm{O} 15$ & $101.3(3)$ & $\mathrm{Cu} 2-\mathrm{Na} 2-\mathrm{W} 3^{\mathrm{ii}}$ & $118.32(15)$ \\
\hline $\mathrm{O} 14-\mathrm{W} 4-\mathrm{O} 21^{\mathrm{i}}$ & $91.5(3)$ & $\mathrm{Cu} 3-\mathrm{Na} 2-\mathrm{W} 3^{\mathrm{ii}}$ & $60.12(9)$ \\
\hline $\mathrm{O} 15-\mathrm{W} 4-\mathrm{O}^{\mathrm{i}}$ & $88.2(3)$ & $\mathrm{Cu} 3-\mathrm{Na} 2-\mathrm{Cu} 2$ & $100.18(13)$ \\
\hline $\mathrm{O} 15-\mathrm{W} 4-07^{\mathrm{i}}$ & $158.6(3)$ & $\mathrm{O} 6-\mathrm{Na} 2-\mathrm{W} 3^{\mathrm{ii}}$ & $101.7(3)$ \\
\hline $\mathrm{O} 15-\mathrm{W} 4-\mathrm{O} 21^{\mathrm{i}}$ & $86.6(3)$ & $\mathrm{O} 6-\mathrm{Na} 2-\mathrm{Cu} 2$ & $93.5(2)$ \\
\hline $\mathrm{O} 21^{\mathrm{i}}-\mathrm{W} 4-\mathrm{O} 4^{\mathrm{i}}$ & $77.3(3)$ & $\mathrm{O} 6-\mathrm{Na} 2-\mathrm{Cu} 3$ & $44.0(2)$ \\
\hline O11-W5-O15 & $81.1(3)$ & $\mathrm{O} 6-\mathrm{Na} 2-\mathrm{O} 10^{\mathrm{ii}}$ & $76.0(3)$ \\
\hline $\mathrm{O} 11-\mathrm{W} 5-\mathrm{O} 18$ & $80.1(3)$ & $\mathrm{O} 6-\mathrm{Na} 2-\mathrm{O} 23$ & $102.4(3)$ \\
\hline $\mathrm{O} 15-\mathrm{W} 5-\mathrm{O} 18$ & $80.2(3)$ & $\mathrm{O} 6-\mathrm{Na} 2-\mathrm{O} 24^{\mathrm{v}}$ & $87.4(3)$ \\
\hline $\mathrm{O} 16-\mathrm{W} 5-\mathrm{O} 11$ & $154.7(3)$ & $\mathrm{O} 6-\mathrm{Na} 2-\mathrm{O} 31$ & $76.2(4)$ \\
\hline $\mathrm{O} 16-\mathrm{W} 5-\mathrm{O} 15$ & $86.7(3)$ & $\mathrm{O} 10^{\mathrm{ii}}-\mathrm{Na} 2-\mathrm{W} 3^{\mathrm{ii}}$ & $26.54(17)$ \\
\hline $\mathrm{O} 16-\mathrm{W} 5-\mathrm{O} 18$ & $76.0(3)$ & $\mathrm{O} 10^{\mathrm{ii}}-\mathrm{Na} 2-\mathrm{Cu} 2$ & $113.4(2)$ \\
\hline $\mathrm{O} 17-\mathrm{W} 5-\mathrm{O} 11$ & $100.9(3)$ & $\mathrm{O} 10^{\mathrm{ii}}-\mathrm{Na} 2-\mathrm{Cu} 3$ & $33.59(18)$ \\
\hline $\mathrm{O} 17-\mathrm{W} 5-\mathrm{O} 15$ & $101.6(3)$ & $\mathrm{O} 10^{\mathrm{ii}}-\mathrm{Na} 2-\mathrm{O} 23$ & $76.4(3)$ \\
\hline $\mathrm{O} 17-\mathrm{W} 5-\mathrm{O} 16$ & $103.2(3)$ & $\mathrm{O} 10^{\mathrm{ii}}-\mathrm{Na} 2-\mathrm{O} 31$ & $123.3(4)$ \\
\hline $\mathrm{O} 17-\mathrm{W} 5-\mathrm{O} 18$ & $178.1(3)$ & $\mathrm{O} 23-\mathrm{Na} 2-\mathrm{W} 3^{\mathrm{ii}}$ & $77.0(2)$ \\
\hline $\mathrm{O} 17-\mathrm{W} 5-\mathrm{O} 19$ & $102.1(3)$ & $\mathrm{O} 23-\mathrm{Na} 2-\mathrm{Cu} 2$ & $41.4(2)$ \\
\hline $\mathrm{O} 19-\mathrm{W} 5-\mathrm{O} 11$ & $89.2(3)$ & $\mathrm{O} 23-\mathrm{Na} 2-\mathrm{Cu} 3$ & $79.5(2)$ \\
\hline $\mathrm{O} 19-\mathrm{W} 5-\mathrm{O} 15$ & $155.7(3)$ & $\mathrm{O} 23-\mathrm{Na} 2-\mathrm{O} 31$ & $158.1(4)$ \\
\hline $\mathrm{O} 19-\mathrm{W} 5-\mathrm{O} 16$ & $93.1(3)$ & $\mathrm{O} 24^{\mathrm{v}}-\mathrm{Na} 2-\mathrm{W} 3^{\mathrm{ii}}$ & $150.1(3)$ \\
\hline $\mathrm{O} 19-\mathrm{W} 5-\mathrm{O} 18$ & $76.2(3)$ & $\mathrm{O} 24^{\mathrm{v}}-\mathrm{Na} 2-\mathrm{Cu} 2$ & $32.0(2)$ \\
\hline 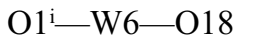 & $72.0(3)$ & $\mathrm{O} 24^{\mathrm{v}}-\mathrm{Na} 2-\mathrm{Cu} 3$ & $115.8(2)$ \\
\hline $\mathrm{O} 1-\mathrm{i}-\mathrm{W} 6-\mathrm{O} 19$ & $81.2(3)$ & $\mathrm{O} 24^{\mathrm{v}}-\mathrm{Na} 2-\mathrm{O} 10^{\mathrm{ii}}$ & $141.2(3)$ \\
\hline $\mathrm{O} 9-\mathrm{W} 6-\mathrm{O} 1^{\mathrm{i}}$ & $154.3(3)$ & $\mathrm{O} 24^{\mathrm{v}}-\mathrm{Na} 2-\mathrm{O} 23$ & $73.3(3)$ \\
\hline $\mathrm{O} 9-\mathrm{W} 6-\mathrm{O} 18$ & $84.3(3)$ & $\mathrm{O} 24^{\mathrm{v}}-\mathrm{Na} 2-\mathrm{O} 31$ & $84.8(4)$ \\
\hline $\mathrm{O} 9-\mathrm{W} 6-\mathrm{O} 19$ & $82.9(3)$ & $\mathrm{O} 31-\mathrm{Na} 2-\mathrm{W} 3^{\mathrm{ii}}$ & $125.0(4)$ \\
\hline $\mathrm{O} 19-\mathrm{W} 6-\mathrm{O} 18$ & $73.4(3)$ & $\mathrm{O} 31-\mathrm{Na} 2-\mathrm{Cu} 2$ & $116.7(4)$ \\
\hline $\mathrm{O} 20-\mathrm{W} 6-\mathrm{O}^{\mathrm{i}}$ & $100.5(3)$ & $\mathrm{O} 31-\mathrm{Na} 2-\mathrm{Cu} 3$ & $111.2(3)$ \\
\hline $\mathrm{O} 20-\mathrm{W} 6-\mathrm{O} 9$ & $100.5(3)$ & $\mathrm{O} 32-\mathrm{Na} 2-\mathrm{W} 3^{\mathrm{ii}}$ & $80.5(3)$ \\
\hline
\end{tabular}




\begin{tabular}{|c|c|c|c|}
\hline $\mathrm{O} 20-\mathrm{W} 6-\mathrm{O} 18$ & $166.0(3)$ & $\mathrm{O} 32-\mathrm{Na} 2-\mathrm{Cu} 2$ & $106.6(3)$ \\
\hline $\mathrm{O} 20-\mathrm{W} 6-\mathrm{O} 19$ & $94.0(3)$ & $\mathrm{O} 32-\mathrm{Na} 2-\mathrm{Cu} 3$ & $139.6(3)$ \\
\hline $\mathrm{O} 20-\mathrm{W} 6-\mathrm{O} 21$ & $102.8(4)$ & $\mathrm{O} 32-\mathrm{Na} 2-\mathrm{O} 6$ & $156.0(4)$ \\
\hline $\mathrm{O} 21-\mathrm{W} 6-\mathrm{O} 1^{\mathrm{i}}$ & $93.7(3)$ & $\mathrm{O} 32-\mathrm{Na} 2-\mathrm{O} 10^{\mathrm{ii}}$ & $106.6(4)$ \\
\hline $\mathrm{O} 21-\mathrm{W} 6-\mathrm{O} 9$ & $95.7(3)$ & $\mathrm{O} 32-\mathrm{Na} 2-\mathrm{O} 23$ & $101.3(4)$ \\
\hline $\mathrm{O} 21-\mathrm{W} 6-\mathrm{O} 18$ & $89.6(3)$ & $\mathrm{O} 32-\mathrm{Na} 2-\mathrm{O} 24^{\mathrm{v}}$ & $102.6(4)$ \\
\hline $\mathrm{O} 21-\mathrm{W} 6-\mathrm{O} 19$ & $163.0(3)$ & $\mathrm{O} 32-\mathrm{Na} 2-\mathrm{O} 31$ & $83.0(4)$ \\
\hline $\mathrm{O} 5^{\mathrm{i}}-\mathrm{Cu} 1-\mathrm{O} 5^{\mathrm{iii}}$ & $180.0(4)$ & $\mathrm{W} 6 \mathrm{i}-\mathrm{O} 1-\mathrm{W} 1$ & $119.2(4)$ \\
\hline $\mathrm{O} 13^{\mathrm{iv}}-\mathrm{Cu} 1-\mathrm{O} 5^{\mathrm{iii}}$ & $95.3(3)$ & $\mathrm{W} 1-\mathrm{O} 2-\mathrm{Na} 1$ & $165.1(4)$ \\
\hline $\mathrm{O} 13-\mathrm{Cu} 1-\mathrm{O} 5^{\mathrm{iii}}$ & $84.7(3)$ & $\mathrm{W} 1-\mathrm{O} 3-\mathrm{W} 3$ & $138.3(4)$ \\
\hline $\mathrm{O} 13-\mathrm{Cu} 1-\mathrm{O} 5^{\mathrm{i}}$ & $95.3(3)$ & $\mathrm{W} 1-\mathrm{O} 4-\mathrm{W} 2$ & $124.5(4)$ \\
\hline $\mathrm{O} 13^{\mathrm{iv}}-\mathrm{Cu} 1-\mathrm{O} 5^{\mathrm{i}}$ & $84.7(3)$ & $\mathrm{W} 1-\mathrm{O} 4-\mathrm{W} 4^{\mathrm{i}}$ & $138.3(4)$ \\
\hline $\mathrm{O} 13-\mathrm{Cu} 1-\mathrm{O} 13^{\mathrm{iv}}$ & 180.0 & $\mathrm{~W} 4-\mathrm{O} 4-\mathrm{W} 2$ & $95.7(3)$ \\
\hline $\mathrm{O} 13^{\mathrm{iv}}-\mathrm{Cu} 1-\mathrm{O} 22^{\mathrm{iv}}$ & $89.8(3)$ & $\mathrm{W} 2-\mathrm{O} 5-\mathrm{Cu} 1^{\mathrm{vi}}$ & $124.8(4)$ \\
\hline $\mathrm{O} 13-\mathrm{Cu} 1-\mathrm{O} 22^{\mathrm{iv}}$ & $90.2(3)$ & $\mathrm{W} 2-\mathrm{O} 6-\mathrm{Cu} 3$ & $128.5(4)$ \\
\hline $\mathrm{O} 13^{\mathrm{iv}}-\mathrm{Cu} 1-\mathrm{O} 22$ & $90.2(3)$ & $\mathrm{W} 2-\mathrm{O} 6-\mathrm{Na} 2$ & $136.6(4)$ \\
\hline $\mathrm{O} 13-\mathrm{Cu} 1-\mathrm{O} 22$ & $89.8(3)$ & $\mathrm{Na} 2-\mathrm{O} 6-\mathrm{Cu} 3$ & $92.4(3)$ \\
\hline $\mathrm{O} 22-\mathrm{Cu} 1-\mathrm{O} 5^{\mathrm{iii}}$ & $96.7(3)$ & $\mathrm{W} 2-\mathrm{O} 7-\mathrm{W} 4^{\mathrm{i}}$ & $116.8(4)$ \\
\hline $\mathrm{O} 22^{\mathrm{iv}}-\mathrm{Cu} 1-\mathrm{O}^{\mathrm{i}}$ & $96.7(3)$ & $\mathrm{W} 2-\mathrm{O} 8-\mathrm{W} 3$ & $118.1(4)$ \\
\hline $\mathrm{O} 22-\mathrm{Cu} 1-\mathrm{O}^{\mathrm{i}}$ & $83.3(3)$ & $\mathrm{W} 3-\mathrm{O} 9-\mathrm{W} 2$ & $93.0(3)$ \\
\hline $\mathrm{O} 22^{\mathrm{iv}}-\mathrm{Cu} 1-\mathrm{O} 5^{\mathrm{iii}}$ & $83.3(3)$ & $\mathrm{W} 6-\mathrm{O} 9-\mathrm{W} 2$ & $124.6(4)$ \\
\hline $\mathrm{O} 22-\mathrm{Cu} 1-\mathrm{O} 22^{\mathrm{iv}}$ & 180.0 & $\mathrm{~W} 6-\mathrm{O} 9-\mathrm{W} 3$ & $140.5(4)$ \\
\hline $\mathrm{Na} 2 \mathrm{v}-\mathrm{Cu} 2-\mathrm{Na} 2$ & 180.0 & $\mathrm{~W} 3-\mathrm{O} 10-\mathrm{Cu} 3$ & $142.8(4)$ \\
\hline $\mathrm{O} 20-\mathrm{Cu} 2-\mathrm{Na} 2^{\mathrm{v}}$ & $89.6(2)$ & $\mathrm{W} 3-\mathrm{O} 10-\mathrm{Na} 2^{\mathrm{ii}}$ & $116.0(4)$ \\
\hline $\mathrm{O} 20-\mathrm{Cu} 2-\mathrm{Na} 2$ & $90.4(2)$ & $\mathrm{Cu} 3-\mathrm{O} 10-\mathrm{Na}_{2}{ }^{\mathrm{ii}}$ & $101.2(3)$ \\
\hline $\mathrm{O} 20^{\mathrm{v}}-\mathrm{Cu} 2-\mathrm{Na} 2^{\mathrm{v}}$ & $90.4(2)$ & W3-O11-W5 & $147.9(4)$ \\
\hline $\mathrm{O} 20^{\mathrm{v}}-\mathrm{Cu} 2-\mathrm{Na} 2$ & $89.6(2)$ & $\mathrm{W} 4-\mathrm{O} 13-\mathrm{Cu} 1$ & $141.2(5)$ \\
\hline $\mathrm{O} 20-\mathrm{Cu} 2-\mathrm{O} 20^{\mathrm{v}}$ & $180.00(15)$ & W4-O15-W5 & $147.1(4)$ \\
\hline $\mathrm{O} 20-\mathrm{Cu} 2-\mathrm{O} 23$ & $90.5(3)$ & $\mathrm{W} 5-\mathrm{O} 16-\mathrm{W} 1^{\mathrm{i}}$ & $115.9(3)$ \\
\hline $\mathrm{O} 20-\mathrm{Cu} 2-\mathrm{O} 23^{\mathrm{v}}$ & $89.5(3)$ & $\mathrm{W} 1 \mathrm{i}-\mathrm{O} 18-\mathrm{W} 5$ & $95.2(3)$ \\
\hline $\mathrm{O} 20^{\mathrm{v}}-\mathrm{Cu} 2-\mathrm{O} 23$ & $89.5(3)$ & $\mathrm{W} 6-\mathrm{O} 18-\mathrm{W} 1^{\mathrm{i}}$ & $96.7(3)$ \\
\hline $\mathrm{O} 20^{\mathrm{v}}-\mathrm{Cu} 2-\mathrm{O} 23^{\mathrm{v}}$ & $90.5(3)$ & W6-O18-W5 & $96.3(3)$ \\
\hline $\mathrm{O} 23^{\mathrm{v}}-\mathrm{Cu} 2-\mathrm{Na} 2^{\mathrm{v}}$ & $45.2(2)$ & W5-O19-W6 & $114.1(4)$ \\
\hline $\mathrm{O} 23^{\mathrm{v}}-\mathrm{Cu} 2-\mathrm{Na} 2$ & $134.8(2)$ & $\mathrm{W} 6-\mathrm{O} 20-\mathrm{Cu} 2$ & $165.2(5)$ \\
\hline $\mathrm{O} 23-\mathrm{Cu} 2-\mathrm{Na} 2$ & $45.2(2)$ & $\mathrm{W} 6-\mathrm{O} 21-\mathrm{W} 4^{\mathrm{i}}$ & $138.5(4)$ \\
\hline $\mathrm{O} 23-\mathrm{Cu} 2-\mathrm{Na} 2^{\mathrm{v}}$ & $134.8(2)$ & $\mathrm{Cu} 2-\mathrm{O} 23-\mathrm{Na} 2$ & $93.5(3)$ \\
\hline $\mathrm{O} 23^{\mathrm{v}}-\mathrm{Cu} 2-\mathrm{O} 23$ & 180.0 & $\mathrm{Cu} 2-\mathrm{O} 24-\mathrm{Na}^{\mathrm{v}}$ & $107.0(4)$ \\
\hline $\mathrm{O} 24-\mathrm{Cu} 2-\mathrm{Na} 2$ & $138.9(2)$ & & \\
\hline
\end{tabular}

Symmetry codes: (i) $-x+1,-y+1,-z+1$; (ii) $-x,-y,-z+1$; (iii) $x, y, z-1$; (iv) $-x+1,-y+1,-z$; (v) $-x+1,-y,-z+1$; (vi) $x, y, z+1$.

(mo_ando241_p-1)

Crystal data

$\mathrm{Na}_{5} \mathrm{Fe}_{2.5}\left[\mathrm{~W}_{12} \mathrm{O}_{40}(\mathrm{OH})_{2}\right] \cdot 36 \mathrm{H}_{2} \mathrm{O}$

$M_{r}=3771.27$

Triclinic, $P \overline{1}$

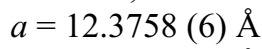

$b=14.7752(7) \AA$

$c=18.8919(8) \AA$

$$
\begin{aligned}
& \alpha=92.9341(14)^{\circ} \\
& \beta=100.6938(14)^{\circ} \\
& \gamma=94.1698(15)^{\circ} \\
& V=3378.1(3) \AA^{3} \\
& Z=2 \\
& F(000)=3388
\end{aligned}
$$


$D_{\mathrm{x}}=3.708 \mathrm{Mg} \mathrm{m}^{-3}$

Mo $K \alpha$ radiation, $\lambda=0.71073 \AA$

Cell parameters from 9822 reflections

$\theta=2.2-25.5^{\circ}$

\section{Data collection}

Bruker D8 Venture

diffractometer

Radiation source: sealed xray tube, Incoatec IuS

$\varphi$ and $\omega$ scans

Absorption correction: multi-scan

(SADABS; Bruker, 2016)

$T_{\min }=0.012, T_{\max }=0.044$

38068 measured reflections

\section{Refinement}

Refinement on $F^{2}$

Least-squares matrix: full

$R\left[F^{2}>2 \sigma\left(F^{2}\right)\right]=0.022$

$w R\left(F^{2}\right)=0.058$

$S=1.09$

12318 reflections

980 parameters

405 restraints

Hydrogen site location: difference Fourier map $\mu=21.02 \mathrm{~mm}^{-1}$

$T=100 \mathrm{~K}$

Needle, clear light orange

$0.37 \times 0.07 \times 0.04 \mathrm{~mm}$

12318 independent reflections

10876 reflections with $I>2 \sigma(I)$

$R_{\text {int }}=0.032$

$\theta_{\text {max }}=25.4^{\circ}, \theta_{\min }=2.3^{\circ}$

$h=-14 \rightarrow 14$

$k=-17 \rightarrow 17$

$l=-22 \rightarrow 22$

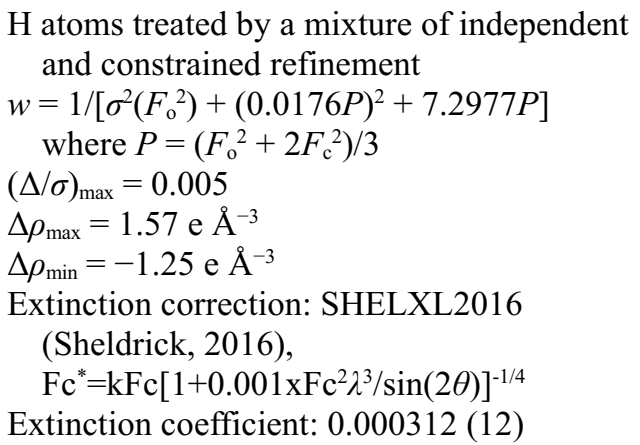

Special details

Geometry. All esds (except the esd in the dihedral angle between two 1.s. planes) are estimated using the full covariance matrix. The cell esds are taken into account individually in the estimation of esds in distances, angles and torsion angles; correlations between esds in cell parameters are only used when they are defined by crystal symmetry. An approximate (isotropic) treatment of cell esds is used for estimating esds involving l.s. planes.

Refinement._olex2_refinement_description

1. Fixed Uiso At 1.5 times of: All $\mathrm{O}(\mathrm{H}, \mathrm{H})$ groups 2. Restrained distances H64A-H61B_\$1 2.2 with sigma of 0.02 H59BH63B 2.2 with sigma of 0.02 H63B_\$2-H71A 2.2 with sigma of 0.02 H71A-H63B_\$2 2.2 with sigma of 0.05 H49B-Na1 2.7 with sigma of $0.01 \mathrm{H} 51 \mathrm{~B}-\mathrm{O} 35$ \$ 42.3 with sigma of $0.04 \mathrm{H} 51 \mathrm{~A}-\mathrm{H} 51 \mathrm{~B} 1.41$ with sigma of $0.01 \mathrm{O} 51-\mathrm{H} 51 \mathrm{~A}=\mathrm{O} 51$ $\mathrm{H} 51 \mathrm{~B} 0.87$ with sigma of $0.01 \mathrm{H} 52 \mathrm{~A}-\mathrm{Na} 32.7$ with sigma of $0.01 \mathrm{O} 52-\mathrm{H} 52 \mathrm{~B}=\mathrm{O} 52-\mathrm{H} 52 \mathrm{~A} 0.87$ with sigma of 0.01 H52A-H52B 1.41 with sigma of $0.01 \mathrm{H} 53 \mathrm{~A}-\mathrm{O} 362$ with sigma of $0.04 \mathrm{H} 53 \mathrm{~A}-\mathrm{H} 53 \mathrm{~B} 1.41$ with sigma of $0.01 \mathrm{O} 53-\mathrm{H} 53 \mathrm{~A}$ $=\mathrm{O} 53-\mathrm{H} 53 \mathrm{~B} 0.87$ with sigma of $0.01 \mathrm{H} 77 \mathrm{~B}-\mathrm{H} 28 \mathrm{~A} 2.2$ with sigma of $0.02 \mathrm{H} 77 \mathrm{~A}-\mathrm{H} 28 \mathrm{~A} 2.2$ with sigma of $0.02 \mathrm{O} 77$ $\mathrm{H} 77 \mathrm{~A}=\mathrm{O} 77-\mathrm{H} 77 \mathrm{~B}=\mathrm{O} 28-\mathrm{H} 28 \mathrm{~B}=\mathrm{O} 28-\mathrm{H} 28 \mathrm{~A} 0.87$ with sigma of $0.01 \mathrm{H} 77 \mathrm{~A}-\mathrm{H} 77 \mathrm{~B}=\mathrm{H} 28 \mathrm{~B}-\mathrm{H} 28 \mathrm{~A} 1.41$ with sigma of $0.01 \mathrm{H} 49 \mathrm{~B}-\mathrm{Na} 22.7$ with sigma of 0.01 Na2-H49A 2.7 with sigma of $0.02 \mathrm{O} 50-\mathrm{H} 50 \mathrm{~A} \sim \mathrm{O} 50-\mathrm{H} 50 \mathrm{~B} \sim \mathrm{O} 51-\mathrm{H} 51 \mathrm{~A} \sim$ O51$\mathrm{H} 51 \mathrm{~B} \sim \mathrm{O} 48-\mathrm{H} 48 \mathrm{~A} \sim \mathrm{O} 48-\mathrm{H} 48 \mathrm{~B} \sim \mathrm{O} 47-\mathrm{H} 47 \mathrm{~B} \mathrm{O} 47-\mathrm{H} 47 \mathrm{~A} \sim \mathrm{O} 65-\mathrm{H} 65 \mathrm{~B} \sim \mathrm{O} 65-\mathrm{H} 65 \mathrm{~A} \sim \mathrm{O} 64-\mathrm{H} 64 \mathrm{~A} \sim \mathrm{O} 64-\mathrm{H} 64 \mathrm{~B} \sim \mathrm{O} 74-$ $\mathrm{H} 74 \mathrm{~A} \sim \mathrm{O} 74-\mathrm{H} 74 \mathrm{~B}$ O63-H63A $\sim$ O63-H63B $\sim$ O60-H60B $\sim$ O60-H60A $\sim$ O167-H16B $\sim$ O167-H16A $\sim$ O67-H67B O67$\mathrm{H} 67 \mathrm{~A} \sim \mathrm{O} 27-\mathrm{H} 27 \mathrm{~B} \sim \mathrm{O} 27-\mathrm{H} 27 \mathrm{~A} \sim \mathrm{O} 28-\mathrm{H} 28 \mathrm{~A} \sim \mathrm{O} 28-\mathrm{H} 28 \mathrm{~B} \sim \mathrm{O} 77-\mathrm{H} 77 \mathrm{~A} \sim \mathrm{O} 77-\mathrm{H} 77 \mathrm{~B}$ with sigma of 0.023 . Others Fixed Sof: O66(0.5) O67(0.5) H67A(0.5) H67B(0.5) O166(0.5) O167(0.5) $\mathrm{H} 16 \mathrm{~A}(0.5) \mathrm{H} 16 \mathrm{~B}(0.5)$ 4.a Free rotating group: O49(H49A,H49B), O62(H62A,H62B), O57(H57A,H57B), O68(H68A,H68B), O69(H69A, H69B), O70(H70A,H70B), O71(H71A,H71B), O72(H72A,H72B), O73(H73A,H73B) 4.b Rotating group: O22(H22A,H22B), O23(H23A,H23B), O24(H24A,H24B), O27(H27A,H27B), O29(H29A, H29B), O47(H47A,H47B), O48(H48A,H48B), O50(H50A,H50B), O54(H54A,H54B), O55(H55A,H55B), O56(H56A,H56B), O58(H58A,H58B), O59(H59A,H59B), O60(H60A,H60B), O61(H61A,H61B), O63(H63A,H63B), O64(H64A,H64B), O65(H65A,H65B), O74(H74A, H74B), O67(H67A,H67B), O167(H16A,H16B) 
Fractional atomic coordinates and isotropic or equivalent isotropic displacement parameters $\left(\AA^{2}\right)$

\begin{tabular}{|c|c|c|c|c|c|}
\hline & $x$ & $y$ & $z$ & $U_{\text {iso }} * / U_{\text {eq }}$ & Occ. $(<1)$ \\
\hline W1 & $0.43982(2)$ & $0.96245(2)$ & $1.17681(2)$ & $0.01193(6)$ & \\
\hline $\mathrm{W} 2$ & $0.25947(2)$ & $0.81700(2)$ & $1.03223(2)$ & $0.01230(6)$ & \\
\hline W3 & $0.39839(2)$ & $0.69804(2)$ & $0.93379(2)$ & $0.01231(6)$ & \\
\hline W4 & $0.61176(2)$ & $0.80885(2)$ & $0.88516(2)$ & $0.01207(6)$ & \\
\hline W5 & $0.34632(2)$ & $0.93167(2)$ & $0.87838(2)$ & $0.01145(5)$ & \\
\hline W6 & $0.41877(2)$ & $1.15417(2)$ & $0.92471(2)$ & $0.01161(6)$ & \\
\hline W7 & $0.09965(2)$ & $0.79501(2)$ & $0.57766(2)$ & $0.01183(6)$ & \\
\hline W8 & $0.24036(2)$ & $0.68517(2)$ & $0.47575(2)$ & $0.01201(6)$ & \\
\hline W9 & $0.15423(2)$ & $0.56265(2)$ & $0.62612(2)$ & $0.01127(5)$ & \\
\hline W10 & $0.08150(2)$ & $0.34469(2)$ & $0.56793(2)$ & $0.01140(6)$ & \\
\hline W11 & $-0.06153(2)$ & $0.45282(2)$ & $0.67587(2)$ & $0.01177(6)$ & \\
\hline W12 & $-0.11444(2)$ & $0.68342(2)$ & $0.62245(2)$ & $0.01181(6)$ & \\
\hline Fe1 & 0.000000 & 1.000000 & 0.500000 & $0.0146(2)$ & \\
\hline $\mathrm{Fe} 2$ & $0.07450(6)$ & $0.82233(5)$ & $0.76386(4)$ & $0.01358(16)$ & \\
\hline $\mathrm{Fe} 3$ & $0.42245(6)$ & $0.66855(5)$ & $0.74623(4)$ & $0.01375(16)$ & \\
\hline $\mathrm{Na} 1$ & 0.500000 & 0.000000 & 0.500000 & $0.0205(7)$ & \\
\hline $\mathrm{Na} 2$ & 0.28394 (19) & $0.13921(14)$ & $0.60840(11)$ & $0.0213(5)$ & \\
\hline $\mathrm{Na} 3$ & 0.50905 (19) & $0.63218(14)$ & $0.41748(11)$ & $0.0193(5)$ & \\
\hline $\mathrm{Na} 4$ & -0.00109 (19) & $1.11946(14)$ & $0.90833(12)$ & $0.0205(5)$ & \\
\hline $\mathrm{Na} 5$ & 0.19312 (19) & $1.34276(14)$ & $0.89244(12)$ & $0.0216(5)$ & \\
\hline $\mathrm{Na} 6$ & 0.000000 & 1.500000 & 1.000000 & $0.0270(7)$ & \\
\hline $\mathrm{O} 75$ & $0.1001(3)$ & $0.8950(2)$ & $0.53226(19)$ & $0.0156(8)$ & \\
\hline $\mathrm{O} 77$ & 0.0108 & $1.0403(3)$ & $0.6088(2)$ & $0.0216(9)$ & \\
\hline H77A & $-0.022(3)$ & $1.079(2)$ & $0.634(2)$ & $0.032 *$ & \\
\hline H77B & $0.0826(10)$ & $1.056(3)$ & $0.6221(19)$ & $0.032 *$ & \\
\hline $\mathrm{O} 1$ & $0.3979(3)$ & $0.9495(2)$ & $1.25833(19)$ & $0.0168(8)$ & \\
\hline $\mathrm{O} 78$ & -0.2529 & $0.7055(2)$ & $0.60234(19)$ & $0.0157(8)$ & \\
\hline $\mathrm{O} 2$ & $0.5723(3)$ & $1.0360(2)$ & 1.20459 (19) & $0.0141(8)$ & \\
\hline $\mathrm{O} 76$ & $0.1546(3)$ & $1.0778(2)$ & 0.49860 (19) & $0.0167(8)$ & \\
\hline $\mathrm{O} 3$ & 0.5139 & $0.8592(2)$ & $1.16577(18)$ & $0.0141(8)$ & \\
\hline $\mathrm{O} 4$ & $0.3706(3)$ & $1.0748(2)$ & 1.14768 (19) & $0.0149(8)$ & \\
\hline $\mathrm{O} 5$ & 0.5025 & $0.9800(2)$ & $1.07220(18)$ & $0.0127(7)$ & \\
\hline O6 & 0.3153 & $0.9044(2)$ & 1.10875 (19) & $0.0137(8)$ & \\
\hline $\mathrm{O} 7$ & 0.2386 & $0.7250(2)$ & $1.08414(19)$ & $0.0177(8)$ & \\
\hline $\mathrm{O} 8$ & $0.4370(3)$ & $0.7922(2)$ & $1.03049(18)$ & $0.0138(8)$ & \\
\hline O9 & 0.3135 & $0.9243(2)$ & $0.96673(19)$ & $0.0144(8)$ & \\
\hline $\mathrm{O} 10$ & 0.1258 & $0.8510(2)$ & $1.00611(19)$ & $0.0162(8)$ & \\
\hline O11 & 0.2618 & $0.7439(2)$ & $0.94416(18)$ & $0.0140(8)$ & \\
\hline $\mathrm{O} 12$ & $0.3941(3)$ & $0.6005(2)$ & 0.98167 (19) & $0.0165(8)$ & \\
\hline $\mathrm{O} 13$ & 0.5521 & $0.7084(2)$ & 0.93601 (19) & $0.0145(8)$ & \\
\hline $\mathrm{O} 14$ & 0.3535 & $0.6564(2)$ & $0.84180(18)$ & $0.0142(8)$ & \\
\hline $\mathrm{O} 15$ & 0.7498 & $0.7843(2)$ & $0.90512(19)$ & $0.0163(8)$ & \\
\hline O16 & $0.5632(3)$ & $0.7473(2)$ & $0.80024(19)$ & $0.0154(8)$ & \\
\hline $\mathrm{O} 17$ & $0.4373(3)$ & $0.8345(2)$ & 0.88711 (19) & $0.0148(8)$ & \\
\hline O18 & $0.3123(3)$ & $1.0590(2)$ & $0.87348(18)$ & $0.0135(7)$ & \\
\hline
\end{tabular}




\begin{tabular}{|c|c|c|c|c|}
\hline O19 & $0.2265(3)$ & $0.8848(2)$ & $0.82087(19)$ & $0.0157(8)$ \\
\hline $\mathrm{O} 20$ & $0.3779(3)$ & $1.1284(2)$ & $1.00996(18)$ & $0.0135(8)$ \\
\hline $\mathrm{O} 21$ & $0.3490(3)$ & $1.2486(2)$ & 0.90007 (19) & $0.0162(8)$ \\
\hline $\mathrm{O} 22$ & $0.4906(3)$ & $0.5437(2)$ & $0.7766(2)$ & $0.0176(8)$ \\
\hline $\mathrm{H} 22 \mathrm{~A}$ & 0.471921 & 0.525896 & 0.818159 & $0.026^{*}$ \\
\hline $\mathrm{H} 22 \mathrm{~B}$ & 0.565119 & 0.549590 & 0.783409 & $0.026^{*}$ \\
\hline $\mathrm{O} 23$ & 0.4817 (3) & $0.6504(2)$ & $0.6488(2)$ & $0.0189(8)$ \\
\hline $\mathrm{H} 23 \mathrm{~A}$ & 0.534097 & 0.610923 & 0.653309 & $0.028^{*}$ \\
\hline H23B & 0.511449 & 0.703254 & 0.636478 & $0.028 *$ \\
\hline $\mathrm{O} 24$ & $0.3712(3)$ & 0.7970 (2) & $0.71232(19)$ & $0.0170(8)$ \\
\hline $\mathrm{H} 24 \mathrm{~A}$ & 0.400970 & 0.812752 & 0.674860 & $0.026^{*}$ \\
\hline $\mathrm{H} 24 \mathrm{~B}$ & 0.392232 & 0.840087 & 0.747703 & $0.026^{*}$ \\
\hline $\mathrm{O} 25$ & $0.2710(3)$ & $0.6072(2)$ & $0.68784(19)$ & $0.0145(8)$ \\
\hline $\mathrm{O} 26$ & 0.1417 (3) & $0.8342(2)$ & $0.67012(19)$ & $0.0163(8)$ \\
\hline $\mathrm{O} 27$ & $0.0118(3)$ & $0.8350(2)$ & 0.85913 (19) & $0.0175(8)$ \\
\hline $\mathrm{H} 27 \mathrm{~A}$ & -0.019350 & 0.780861 & 0.870617 & $0.026^{*}$ \\
\hline H27B & -0.041858 & 0.874914 & 0.855558 & $0.026^{*}$ \\
\hline O28 & $0.0044(3)$ & 0.9491 (2) & 0.73617 (19) & $0.0171(8)$ \\
\hline $\mathrm{H} 28 \mathrm{~A}$ & $0.025(4)$ & $0.961(3)$ & $0.6950(14)$ & $0.026^{*}$ \\
\hline $\mathrm{H} 28 \mathrm{~B}$ & $0.025(5)$ & 0.999 (2) & $0.7650(19)$ & $0.026^{*}$ \\
\hline O29 & $0.1294(3)$ & 0.6937 (2) & $0.7944(2)$ & $0.0171(8)$ \\
\hline $\mathrm{H} 29 \mathrm{~A}$ & 0.120845 & 0.654521 & 0.755798 & $0.026^{*}$ \\
\hline $\mathrm{H} 29 \mathrm{~B}$ & 0.090838 & 0.670087 & 0.825449 & $0.026^{*}$ \\
\hline O30 & $-0.0686(3)$ & $0.7429(2)$ & $0.70806(19)$ & $0.0173(8)$ \\
\hline $\mathrm{O} 31$ & $-0.0565(3)$ & 0.7844 (2) & $0.57317(19)$ & $0.0130(7)$ \\
\hline $\mathrm{O} 32$ & $0.0612(3)$ & 0.6594 (2) & $0.62006(19)$ & $0.0152(8)$ \\
\hline $\mathrm{O} 33$ & $0.0626(3)$ & $0.7070(2)$ & $0.47893(19)$ & $0.0138(8)$ \\
\hline $\mathrm{O} 34$ & 0.1905 (3) & $0.5774(2)$ & $0.53939(19)$ & $0.0158(8)$ \\
\hline $\mathrm{O} 35$ & $0.2371(3)$ & $0.7542(2)$ & $0.56669(18)$ & $0.0149(8)$ \\
\hline $\mathrm{O} 36$ & 0.2569 (3) & 0.7839 (2) & $0.42933(19)$ & $0.0150(8)$ \\
\hline $\mathrm{O} 37$ & $0.3757(3)$ & $0.6545(2)$ & $0.49892(19)$ & $0.0163(8)$ \\
\hline $\mathrm{O} 38$ & $-0.1295(3)$ & $0.5646(2)$ & $0.65250(19)$ & $0.0146(8)$ \\
\hline O39 & $-0.1851(3)$ & 0.3951 (2) & $0.60394(19)$ & $0.0137(7)$ \\
\hline $\mathrm{O} 40$ & $-0.1030(3)$ & 0.4288 (2) & $0.7553(2)$ & $0.0177(8)$ \\
\hline O41 & $0.0140(3)$ & $0.3492(2)$ & $0.65924(18)$ & $0.0134(7)$ \\
\hline $\mathrm{O} 42$ & $0.0032(3)$ & $0.4785(2)$ & $0.57249(18)$ & $0.0134(8)$ \\
\hline $\mathrm{O} 43$ & $0.0721(3)$ & $0.5233(2)$ & 0.70755 (19) & $0.0143(8)$ \\
\hline $\mathrm{O} 44$ & $-0.1231(3)$ & $0.6218(2)$ & $0.51595(18)$ & $0.0128(7)$ \\
\hline $\mathrm{O} 45$ & $0.1879(3)$ & $0.4362(2)$ & $0.62501(18)$ & $0.0134(7)$ \\
\hline $\mathrm{O} 46$ & $0.1513(3)$ & $0.2498(2)$ & $0.58872(19)$ & $0.0157(8)$ \\
\hline O47 & 0.3478 (3) & 0.2254 (2) & $0.7211(2)$ & $0.0203(9)$ \\
\hline H47A & 0.376148 & 0.281108 & 0.714149 & $0.030 *$ \\
\hline H47B & 0.400373 & 0.197678 & 0.748989 & $0.030^{*}$ \\
\hline O48 & $0.2635(4)$ & $0.0174(3)$ & 0.6787 (2) & $0.0340(11)$ \\
\hline H48A & 0.329102 & 0.003969 & 0.702825 & $0.051 *$ \\
\hline H48B & 0.232193 & -0.032050 & 0.651118 & $0.051^{*}$ \\
\hline O49 & $0.4680(4)$ & $0.0895(3)$ & $0.6048(2)$ & $0.0229(9)$ \\
\hline $\mathrm{H} 49 \mathrm{~A}$ & 0.505130 & 0.135577 & 0.631042 & $0.034 *$ \\
\hline
\end{tabular}




\begin{tabular}{|c|c|c|c|c|}
\hline H49B & 0.466632 & 0.046477 & 0.634452 & $0.034 *$ \\
\hline $\mathrm{O} 50$ & 0.7003 & $0.0214(2)$ & $0.5578(2)$ & $0.0216(9)$ \\
\hline H50A & 0.709887 & 0.009412 & 0.604986 & $0.032 *$ \\
\hline H50B & 0.727030 & 0.080448 & 0.559699 & $0.032 *$ \\
\hline O51 & $0.5203(4)$ & $0.1320(3)$ & $0.4330(2)$ & $0.0265(9)$ \\
\hline H51A & $0.478(4)$ & $0.139(4)$ & $0.3902(16)$ & $0.040 *$ \\
\hline H51B & $0.5888(17)$ & $0.150(4)$ & $0.427(3)$ & $0.040 *$ \\
\hline O52 & $0.6197(4)$ & $0.7649(2)$ & $0.4778(2)$ & $0.0215(9)$ \\
\hline H52A & $0.663(3)$ & $0.7394(10)$ & $0.5110(18)$ & $0.032 *$ \\
\hline H52B & $0.577(4)$ & $0.797(4)$ & $0.499(2)$ & $0.032 *$ \\
\hline $\mathrm{O} 53$ & $0.4156(3)$ & $0.7413(3)$ & $0.3450(2)$ & $0.0232(9)$ \\
\hline H53A & $0.355(2)$ & $0.745(4)$ & $0.362(2)$ & $0.035 *$ \\
\hline H53B & $0.397(4)$ & $0.728(4)$ & $0.2988(8)$ & $0.035 *$ \\
\hline O54 & $0.6019(3)$ & $0.6050(2)$ & $0.32011(19)$ & $0.0185(8)$ \\
\hline H54A & 0.569316 & 0.556728 & 0.292040 & $0.028 *$ \\
\hline H54B & 0.671969 & 0.594865 & 0.336455 & $0.028^{*}$ \\
\hline $\mathrm{O} 55$ & $0.6443(3)$ & $0.5476(2)$ & $0.4892(2)$ & $0.0193(8)$ \\
\hline H55A & 0.713115 & 0.572858 & 0.490711 & $0.029 *$ \\
\hline H55B & 0.640827 & 0.489651 & 0.470739 & $0.029 *$ \\
\hline O56 & 0.4026 & $0.4899(2)$ & $0.3769(2)$ & $0.0205(8)$ \\
\hline H56A & 0.351594 & 0.497092 & 0.338263 & $0.031 *$ \\
\hline H56B & 0.369101 & 0.469394 & 0.411358 & $0.031 *$ \\
\hline O58 & $0.1426(3)$ & $1.0451(2)$ & $0.9812(2)$ & $0.0220(9)$ \\
\hline H58A & 0.208095 & 1.069437 & 0.976634 & $0.033 *$ \\
\hline H58B & 0.137172 & 0.986597 & 0.967312 & $0.033^{*}$ \\
\hline O59 & 0.1030 & $1.0982(2)$ & $0.8154(2)$ & $0.0196(8)$ \\
\hline H59A & 0.172924 & 1.082290 & 0.833307 & $0.029 *$ \\
\hline Н59B & 0.111317 & 1.150360 & 0.791637 & $0.029 *$ \\
\hline $\mathrm{O} 60$ & $-0.1092(3)$ & $0.9738(2)$ & $0.8820(2)$ & $0.0231(9)$ \\
\hline H60A & -0.166090 & 0.977993 & 0.846658 & $0.035^{*}$ \\
\hline H60B & -0.134022 & 0.956833 & 0.920654 & $0.035 *$ \\
\hline O61 & $0.3140(3)$ & $1.4191(2)$ & $0.9940(2)$ & $0.0229(9)$ \\
\hline H61A & 0.369086 & 1.458527 & 0.980717 & $0.034 *$ \\
\hline H61B & 0.352070 & 1.378925 & 1.025227 & $0.034 *$ \\
\hline O62 & $0.1017(4)$ & $1.2528(3)$ & $0.9709(2)$ & $0.0218(9)$ \\
\hline H62A & 0.145760 & 1.229523 & 1.005333 & $0.033 *$ \\
\hline H62B & 0.060640 & 1.285112 & 0.993112 & $0.033 *$ \\
\hline O63 & $0.1595(3)$ & $1.2692(2)$ & $0.7723(2)$ & $0.0204(8)$ \\
\hline H63A & 0.214911 & 1.286341 & 0.744070 & $0.031 *$ \\
\hline H63B & 0.090044 & 1.282058 & 0.742511 & $0.031 *$ \\
\hline O64 & $-0.1812(4)$ & $1.4738(3)$ & $0.9349(2)$ & $0.0286(10)$ \\
\hline H64A & -0.234836 & 1.515714 & 0.949155 & $0.043^{*}$ \\
\hline H64B & -0.218263 & 1.412264 & 0.938246 & $0.043 *$ \\
\hline O65 & $-0.0222(4)$ & $1.3617(3)$ & $1.0594(2)$ & $0.0269(9)$ \\
\hline H65A & -0.092383 & 1.350512 & 1.064955 & $0.040^{*}$ \\
\hline H65B & 0.021278 & 1.365054 & 1.103450 & $0.040 *$ \\
\hline $\mathrm{O} 74$ & $0.2373(4)$ & $1.4742(3)$ & $0.8282(2)$ & $0.0339(11)$ \\
\hline H74A & 0.277054 & 1.459042 & 0.795994 & $0.051^{*}$ \\
\hline
\end{tabular}




\begin{tabular}{|c|c|c|c|c|c|}
\hline H74B & 0.275256 & 1.516954 & 0.859008 & $0.051^{*}$ & \\
\hline O66 & $0.0455(9)$ & $1.4372(6)$ & $0.8932(6)$ & $0.025(2)$ & 0.5 \\
\hline O67 & $-0.0980(10)$ & $1.2057(8)$ & $0.8245(6)$ & $0.040(3)$ & 0.5 \\
\hline H67A & -0.172297 & 1.223060 & 0.834440 & $0.061^{*}$ & 0.5 \\
\hline H67B & -0.118922 & 1.176966 & 0.772875 & $0.061^{*}$ & 0.5 \\
\hline O166 & $0.0109(11)$ & $1.3927(8)$ & $0.8881(6)$ & $0.036(3)$ & 0.5 \\
\hline O167 & $-0.1337(10)$ & $1.1537(9)$ & $0.8069(7)$ & $0.047(3)$ & 0.5 \\
\hline H16A & -0.120091 & 1.218036 & 0.783218 & $0.070^{*}$ & 0.5 \\
\hline H16B & -0.219353 & 1.157915 & 0.813213 & $0.070 *$ & 0.5 \\
\hline O57 & $0.3650(4)$ & $0.6621(3)$ & $0.2063(2)$ & $0.0269(9)$ & \\
\hline H57A & 0.426675 & 0.650088 & 0.193605 & $0.040^{*}$ & \\
\hline H57B & 0.322656 & 0.684798 & 0.170648 & $0.040^{*}$ & \\
\hline O68 & $0.8504(4)$ & $0.6607(2)$ & $0.8242(2)$ & $0.0222(9)$ & \\
\hline H68A & 0.874753 & 0.686576 & 0.789231 & $0.033^{*}$ & \\
\hline H68B & 0.811052 & 0.697443 & 0.843819 & $0.033^{*}$ & \\
\hline O69 & 0.6958 & $0.5233(3)$ & $0.7662(2)$ & $0.0230(9)$ & \\
\hline H69A & 0.706601 & 0.467755 & 0.777240 & $0.034^{*}$ & \\
\hline H69B & 0.749060 & 0.560225 & 0.790897 & $0.034 *$ & \\
\hline $\mathrm{O} 70$ & $0.4874(4)$ & $0.4128(3)$ & $0.8762(2)$ & $0.0258(9)$ & \\
\hline H70A & 0.526630 & 0.411288 & 0.919288 & $0.039 *$ & \\
\hline H70B & 0.422232 & 0.385367 & 0.874613 & $0.039 *$ & \\
\hline O71 & $0.9183(3)$ & 0.1787 (2) & $0.6740(2)$ & $0.0227(9)$ & \\
\hline H71A & 0.945097 & 0.231566 & 0.664001 & $0.034^{*}$ & \\
\hline H71B & 0.851249 & 0.167453 & 0.649725 & $0.034 *$ & \\
\hline $\mathrm{O} 72$ & $0.7852(4)$ & $0.9728(3)$ & $0.7021(2)$ & $0.0282(10)$ & \\
\hline $\mathrm{H} 72 \mathrm{~A}$ & 0.741468 & 0.923175 & 0.697101 & $0.042 *$ & \\
\hline H72B & 0.751210 & 1.018193 & 0.715589 & $0.042 *$ & \\
\hline $\mathrm{O} 73$ & $0.6434(4)$ & $0.8229(2)$ & $0.6820(2)$ & $0.0211(9)$ & \\
\hline H73A & 0.614540 & 0.802804 & 0.717447 & $0.032 *$ & \\
\hline H73B & 0.681198 & 0.781510 & 0.666241 & $0.032 *$ & \\
\hline
\end{tabular}

Atomic displacement parameters $\left(\AA^{2}\right)$

\begin{tabular}{lllllll}
\hline & $U^{11}$ & $U^{22}$ & $U^{33}$ & $U^{12}$ & $U^{13}$ & $U^{23}$ \\
\hline W1 & $0.01172(12)$ & $0.01254(10)$ & $0.01175(11)$ & $0.00075(8)$ & $0.00268(8)$ & $0.00154(8)$ \\
W2 & $0.01205(12)$ & $0.01225(10)$ & $0.01259(11)$ & $0.00030(8)$ & $0.00248(8)$ & $0.00132(8)$ \\
W3 & $0.01267(12)$ & $0.01148(10)$ & $0.01268(11)$ & $0.00047(8)$ & $0.00234(8)$ & $0.00094(8)$ \\
W4 & $0.01198(12)$ & $0.01195(10)$ & $0.01232(11)$ & $0.00099(8)$ & $0.00236(8)$ & $0.00104(8)$ \\
W5 & $0.01123(12)$ & $0.01134(10)$ & $0.01177(10)$ & $0.00078(8)$ & $0.00211(8)$ & $0.00128(8)$ \\
W6 & $0.01164(12)$ & $0.01129(10)$ & $0.01178(10)$ & $0.00095(8)$ & $0.00183(8)$ & $0.00125(8)$ \\
W7 & $0.01197(12)$ & $0.01111(10)$ & $0.01233(10)$ & $0.00082(8)$ & $0.00213(8)$ & $0.00099(8)$ \\
W8 & $0.01164(12)$ & $0.01182(10)$ & $0.01258(11)$ & $0.00048(8)$ & $0.00245(8)$ & $0.00124(8)$ \\
W9 & $0.01105(12)$ & $0.01091(10)$ & $0.01184(10)$ & $0.00080(8)$ & $0.00205(8)$ & $0.00127(8)$ \\
W10 & $0.01133(12)$ & $0.01107(10)$ & $0.01177(10)$ & $0.00089(8)$ & $0.00202(8)$ & $0.00114(8)$ \\
W11 & $0.01170(12)$ & $0.01198(10)$ & $0.01187(11)$ & $0.00078(8)$ & $0.00277(8)$ & $0.00146(8)$ \\
W12 & $0.01179(12)$ & $0.01161(10)$ & $0.01209(11)$ & $0.00098(8)$ & $0.00230(8)$ & $0.00121(8)$ \\
Fe1 & $0.0158(6)$ & $0.0140(5)$ & $0.0141(5)$ & $0.0019(4)$ & $0.0030(4)$ & $0.0021(4)$ \\
Fe2 & $0.0130(4)$ & $0.0142(3)$ & $0.0132(4)$ & $0.0001(3)$ & $0.0022(3)$ & $0.0005(3)$
\end{tabular}




\begin{tabular}{|c|c|c|c|c|c|c|}
\hline $\mathrm{Fe} 3$ & $0.0133(4)$ & $0.0137(3)$ & $0.0140(4)$ & $-0.0001(3)$ & $0.0023(3)$ & $0.0005(3)$ \\
\hline $\mathrm{Na} 1$ & $0.0215(18)$ & $0.0183(15)$ & $0.0213(16)$ & $0.0016(13)$ & $0.0035(13)$ & $0.0010(12)$ \\
\hline $\mathrm{Na} 2$ & $0.0235(13)$ & $0.0208(10)$ & $0.0191(11)$ & $0.0053(10)$ & $0.0010(9)$ & $0.0018(9)$ \\
\hline $\mathrm{Na} 3$ & $0.0198(13)$ & $0.0191(10)$ & $0.0198(11)$ & $0.0021(9)$ & 0.0057 (9) & $0.0023(9)$ \\
\hline $\mathrm{Na} 4$ & $0.0204(13)$ & $0.0220(11)$ & $0.0208(11)$ & $0.0027(9)$ & $0.0074(9)$ & $0.0044(9)$ \\
\hline $\mathrm{Na} 5$ & $0.0222(13)$ & $0.0223(11)$ & $0.0206(11)$ & $0.0046(10)$ & $0.0032(9)$ & $0.0024(9)$ \\
\hline $\mathrm{Na} 6$ & $0.0214(19)$ & $0.0287(17)$ & $0.0322(19)$ & $0.0043(15)$ & $0.0050(15)$ & $0.0118(14)$ \\
\hline $\mathrm{O} 75$ & $0.014(2)$ & $0.0130(17)$ & $0.0200(19)$ & $0.0037(15)$ & $0.0038(15)$ & $0.0025(14)$ \\
\hline O77 & $0.019(2)$ & $0.025(2)$ & $0.020(2)$ & 0.0053 (17) & 0.0017 (17) & $-0.0017(16)$ \\
\hline $\mathrm{O} 1$ & $0.017(2)$ & $0.0177(18)$ & $0.0156(19)$ & $-0.0001(16)$ & $0.0028(15)$ & $-0.0009(15)$ \\
\hline $\mathrm{O} 78$ & $0.014(2)$ & $0.0158(17)$ & $0.0178(19)$ & $0.0008(15)$ & $0.0033(15)$ & 0.0003 (14) \\
\hline $\mathrm{O} 2$ & $0.013(2)$ & $0.0142(17)$ & $0.0142(18)$ & $-0.0001(15)$ & $0.0011(15)$ & $0.0016(14)$ \\
\hline O76 & $0.017(2)$ & $0.0150(17)$ & $0.0184(19)$ & $0.0002(15)$ & $0.0045(16)$ & $0.0013(15)$ \\
\hline $\mathrm{O} 3$ & $0.016(2)$ & $0.0140(17)$ & $0.0128(18)$ & $0.0000(15)$ & $0.0045(15)$ & $0.0042(14)$ \\
\hline $\mathrm{O} 4$ & $0.015(2)$ & $0.0136(17)$ & $0.0171(19)$ & $0.0022(15)$ & $0.0052(15)$ & 0.0009 (14) \\
\hline O5 & $0.013(2)$ & $0.0111(16)$ & $0.0135(18)$ & $-0.0014(15)$ & $0.0024(15)$ & $-0.0003(14)$ \\
\hline O6 & $0.012(2)$ & $0.0143(17)$ & $0.0159(18)$ & $0.0033(15)$ & $0.0039(15)$ & $0.0011(14)$ \\
\hline $\mathrm{O} 7$ & $0.021(2)$ & $0.0144(17)$ & $0.0174(19)$ & $-0.0015(16)$ & $0.0046(16)$ & $0.0022(15)$ \\
\hline O8 & $0.014(2)$ & $0.0127(17)$ & $0.0147(18)$ & $0.0021(15)$ & $0.0019(15)$ & $0.0028(14)$ \\
\hline O9 & $0.016(2)$ & $0.0135(17)$ & $0.0138(18)$ & $0.0016(15)$ & $0.0025(15)$ & $0.0016(14)$ \\
\hline $\mathrm{O} 10$ & $0.015(2)$ & $0.0137(17)$ & $0.0184(19)$ & $-0.0022(15)$ & $0.0012(15)$ & $-0.0007(14)$ \\
\hline O11 & $0.011(2)$ & $0.0149(17)$ & $0.0148(18)$ & $-0.0024(15)$ & $0.0003(15)$ & $-0.0010(14)$ \\
\hline O12 & $0.018(2)$ & $0.0147(17)$ & 0.0169 (19) & $0.0038(16)$ & 0.0033 (16) & $0.0023(14)$ \\
\hline $\mathrm{O} 13$ & $0.012(2)$ & $0.0163(17)$ & $0.0146(18)$ & $0.0023(15)$ & $0.0002(15)$ & $0.0003(14)$ \\
\hline O14 & $0.015(2)$ & $0.0183(18)$ & 0.0095 (17) & $0.0022(15)$ & $0.0027(14)$ & $0.0003(14)$ \\
\hline O15 & $0.014(2)$ & $0.0161(18)$ & $0.0182(19)$ & $0.0009(15)$ & $0.0013(15)$ & $0.0007(15)$ \\
\hline O16 & $0.016(2)$ & $0.0160(17)$ & $0.0133(18)$ & $-0.0009(15)$ & $0.0020(15)$ & $-0.0003(14)$ \\
\hline O17 & $0.014(2)$ & $0.0129(17)$ & $0.0180(19)$ & $0.0029(15)$ & $0.0039(15)$ & $0.0044(14)$ \\
\hline $\mathrm{O} 18$ & $0.013(2)$ & $0.0138(17)$ & $0.0147(18)$ & $0.0038(15)$ & $0.0028(15)$ & $0.0027(14)$ \\
\hline O19 & $0.018(2)$ & $0.0138(17)$ & $0.0160(19)$ & $0.0027(15)$ & $0.0029(15)$ & $0.0021(14)$ \\
\hline $\mathrm{O} 20$ & $0.014(2)$ & $0.0128(17)$ & $0.0136(18)$ & $-0.0009(15)$ & $0.0037(15)$ & $-0.0009(14)$ \\
\hline $\mathrm{O} 21$ & $0.016(2)$ & $0.0149(17)$ & $0.0171(19)$ & $0.0007(15)$ & $0.0018(15)$ & $0.0016(15)$ \\
\hline $\mathrm{O} 22$ & $0.013(2)$ & $0.0175(18)$ & $0.021(2)$ & $0.0005(16)$ & $0.0000(16)$ & $0.0004(15)$ \\
\hline $\mathrm{O} 23$ & $0.017(2)$ & $0.0179(18)$ & $0.023(2)$ & $0.0025(16)$ & $0.0065(16)$ & $0.0014(15)$ \\
\hline $\mathrm{O} 24$ & $0.022(2)$ & $0.0154(18)$ & $0.0125(18)$ & $0.0026(16)$ & $0.0007(16)$ & $0.0007(14)$ \\
\hline $\mathrm{O} 25$ & $0.016(2)$ & $0.0141(17)$ & $0.0139(18)$ & $0.0022(15)$ & $0.0029(15)$ & $0.0005(14)$ \\
\hline $\mathrm{O} 26$ & $0.014(2)$ & $0.0159(17)$ & $0.0192(19)$ & $0.0007(15)$ & $0.0035(15)$ & $0.0003(15)$ \\
\hline $\mathrm{O} 27$ & $0.017(2)$ & $0.0180(18)$ & $0.0181(19)$ & $0.0028(16)$ & $0.0035(16)$ & $0.0023(15)$ \\
\hline $\mathrm{O} 28$ & $0.020(2)$ & $0.0147(17)$ & $0.0152(19)$ & $-0.0001(16)$ & $0.0015(16)$ & $-0.0009(15)$ \\
\hline O29 & $0.020(2)$ & $0.0152(18)$ & $0.0157(19)$ & $0.0030(16)$ & $0.0017(16)$ & $-0.0011(14)$ \\
\hline $\mathrm{O} 30$ & $0.018(2)$ & $0.0194(18)$ & $0.0145(19)$ & $0.0013(16)$ & $0.0019(15)$ & $0.0023(15)$ \\
\hline $\mathrm{O} 31$ & $0.0106(19)$ & $0.0127(16)$ & $0.0159(18)$ & $0.0025(15)$ & $0.0019(14)$ & $0.0028(14)$ \\
\hline $\mathrm{O} 32$ & $0.017(2)$ & $0.0133(17)$ & $0.0163(19)$ & $0.0012(15)$ & $0.0047(15)$ & $0.0034(14)$ \\
\hline $\mathrm{O} 33$ & $0.014(2)$ & $0.0131(17)$ & $0.0152(18)$ & $0.0025(15)$ & $0.0036(15)$ & $0.0031(14)$ \\
\hline $\mathrm{O} 34$ & $0.018(2)$ & $0.0128(17)$ & $0.0180(19)$ & $0.0031(15)$ & $0.0075(16)$ & $0.0005(14)$ \\
\hline $\mathrm{O} 35$ & $0.012(2)$ & $0.0174(18)$ & $0.0143(18)$ & $-0.0024(15)$ & $0.0029(15)$ & $-0.0012(14)$ \\
\hline $\mathrm{O} 36$ & $0.018(2)$ & $0.0109(17)$ & $0.0161(18)$ & $-0.0002(15)$ & $0.0039(15)$ & $0.0011(14)$ \\
\hline $\mathrm{O} 37$ & 0.014 (2) & $0.0140(17)$ & 0.0188 (19) & $-0.0014(15)$ & $-0.0003(15)$ & $-0.0019(14)$ \\
\hline
\end{tabular}




\begin{tabular}{|c|c|c|c|c|c|c|}
\hline $\mathrm{O} 38$ & $0.011(2)$ & $0.0153(17)$ & $0.0182(19)$ & $0.0011(15)$ & $0.0034(15)$ & $0.0029(14)$ \\
\hline O39 & $0.013(2)$ & $0.0149(17)$ & $0.0137(18)$ & $0.0044(15)$ & $0.0017(15)$ & $0.0020(14)$ \\
\hline $\mathrm{O} 40$ & $0.016(2)$ & $0.0197(18)$ & $0.0183(19)$ & $0.0029(16)$ & $0.0049(16)$ & $0.0037(15)$ \\
\hline O41 & $0.014(2)$ & $0.0143(17)$ & $0.0115(17)$ & $0.0004(15)$ & $0.0025(15)$ & $0.0025(14)$ \\
\hline $\mathrm{O} 42$ & $0.016(2)$ & $0.0116(16)$ & $0.0119(17)$ & $-0.0014(15)$ & $0.0004(15)$ & $0.0010(14)$ \\
\hline $\mathrm{O} 43$ & $0.013(2)$ & $0.0158(17)$ & $0.0140(18)$ & $0.0013(15)$ & $0.0023(15)$ & $-0.0011(14)$ \\
\hline $\mathrm{O} 44$ & 0.0098 (19) & $0.0121(16)$ & $0.0155(18)$ & $-0.0009(14)$ & $0.0016(14)$ & -0.0020 \\
\hline $\mathrm{O} 45$ & $0.012(2)$ & $0.0141(17)$ & $0.0145(18)$ & $0.0036(15)$ & $0.0038(15)$ & $0.0017(14)$ \\
\hline $\mathrm{O} 46$ & 0.015 & $0.0143(17)$ & $0.0172(19)$ & $0.0001(15)$ & $0.0023(15)$ & $0.0014(14)$ \\
\hline O47 & $0.023(2)$ & $0.0176(18)$ & $0.020(2)$ & $0.0025(17)$ & $0.0021(17)$ & $0.0033(15)$ \\
\hline O48 & $0.032(3)$ & $0.027(2)$ & 0.035 & $-0.010(2)$ & $-0.013(2)$ & $0.0112(19)$ \\
\hline O49 & $0.029(3)$ & $0.0205(19)$ & $0.019(2)$ & $0.0028(18)$ & $0.0034(17)$ & $0.0034(16)$ \\
\hline $\mathrm{O} 50$ & $0.023(2)$ & $0.0148(18)$ & $0.026(2)$ & $0.0003(17)$ & $0.0049(18)$ & $-0.0003(16)$ \\
\hline O51 & $0.026(2)$ & $0.027(2)$ & $0.027(2)$ & $-0.0014(19)$ & $0.0041(18)$ & $0.0060(18)$ \\
\hline O52 & $0.023(2)$ & 0.0185 (19) & $0.021(2)$ & $0.0062(17)$ & $-0.0009(17)$ & $0.0043(16)$ \\
\hline $\mathrm{O} 53$ & $0.021(2)$ & $0.030(2)$ & $0.021(2)$ & $0.0054(18)$ & $0.0070(17)$ & $0.0020(18)$ \\
\hline O54 & 0.019 (2) & $0.0185(18)$ & $0.0167(19)$ & $0.0018(16)$ & $0.0011(16)$ & $0.0002(15)$ \\
\hline O55 & 0.019 (2) & $0.0170(18)$ & $0.022(2)$ & $0.0009(16)$ & $0.0042(17)$ & $0.0041(16)$ \\
\hline O56 & $0.020(2)$ & 0.0204 (19) & $0.021(2)$ & $-0.0001(17)$ & $0.0053(17)$ & $0.0002(16)$ \\
\hline $\mathrm{O} 58$ & $0.022(2)$ & $0.0171(19)$ & $0.030(2)$ & $0.0059(17)$ & $0.0077(18)$ & $0.0077(16)$ \\
\hline O59 & $0.020(2)$ & $0.0163(18)$ & $0.022(2)$ & $0.0026(16)$ & $0.0018(17)$ & $0.0020(15)$ \\
\hline O60 & $0.021(2)$ & $0.023(2)$ & $0.024(2)$ & $0.0038(17)$ & $0.0026(17)$ & $0.0005(17)$ \\
\hline O61 & $0.027(2)$ & $0.0155(18)$ & $0.026(2)$ & $0.0017(17)$ & $0.0041(18)$ & $0.0049(16)$ \\
\hline O62 & $0.021(2)$ & $0.021(2)$ & $0.023(2)$ & $0.0034(17)$ & $0.0019(17)$ & $0.0039(16)$ \\
\hline O63 & $0.019(2)$ & $0.0216(19)$ & $0.020(2)$ & $0.0010(17)$ & $0.0016(16)$ & $0.0040(16)$ \\
\hline O64 & $0.027(3)$ & $0.018(2)$ & $0.042(3)$ & $0.0026(18)$ & $0.009(2)$ & $0.0024(18)$ \\
\hline O65 & $0.026(2)$ & $0.030(2)$ & $0.025(2)$ & $0.0000(19)$ & $0.0054(18)$ & $0.0091(18)$ \\
\hline O74 & $0.044(3)$ & $0.026(2)$ & $0.026(2)$ & $-0.008(2)$ & $-0.003(2)$ & $0.0047(18)$ \\
\hline O66 & $0.024(6)$ & $0.024(5)$ & $0.025(5)$ & $0.003(4)$ & $0.001(4)$ & $0.002(5)$ \\
\hline O67 & $0.030(7)$ & $0.075(9)$ & $0.028(6)$ & $0.033(6)$ & $0.017(5)$ & $0.027(6)$ \\
\hline O166 & $0.050(8)$ & $0.043(7)$ & $0.021(5)$ & $0.025(6)$ & $0.011(5)$ & $0.011(5)$ \\
\hline O167 & $0.029(7)$ & $0.079(9)$ & $0.042(7)$ & $0.013(6)$ & $0.021(6)$ & $0.033(7)$ \\
\hline O57 & $0.034(3)$ & $0.027(2)$ & 0.019 (2) & $0.008(2)$ & $0.0013(18)$ & 0.0053 (17) \\
\hline O68 & $0.025(2)$ & $0.0220(19)$ & $0.022(2)$ & $-0.0006(18)$ & $0.0110(17)$ & $0.0005(16)$ \\
\hline O69 & $0.017(2)$ & 0.0207 (19) & $0.029(2)$ & $-0.0010(17)$ & $0.0008(17)$ & 0.0005 (17) \\
\hline $\mathrm{O} 70$ & $0.025(3)$ & $0.031(2)$ & $0.020(2)$ & -0.0015 (19) & $-0.0007(17)$ & $0.0079(18)$ \\
\hline O71 & $0.023(2)$ & $0.0154(18)$ & $0.028(2)$ & $-0.0029(17)$ & 0.0007 (18) & $0.0046(16)$ \\
\hline $\mathrm{O} 72$ & $0.024(3)$ & $0.021(2)$ & $0.041(3)$ & $0.0019(18)$ & $0.011(2)$ & $-0.0005(19)$ \\
\hline $\mathrm{O} 73$ & $0.026(2)$ & $0.0209(19)$ & $0.019(2)$ & $0.0026(18)$ & $0.0106(17)$ & $0.0032(16)$ \\
\hline
\end{tabular}

Geometric parameters $(\AA, \stackrel{\circ}{)})$

\begin{tabular}{llll}
\hline $\mathrm{W} 1-\mathrm{O} 1$ & $1.729(4)$ & $\mathrm{Fe} 1-\mathrm{O} 77^{\mathrm{iii}}$ & $2.087(4)$ \\
$\mathrm{W} 1-\mathrm{O} 2$ & $1.873(4)$ & $\mathrm{Fe} 1-\mathrm{O} 77$ & $2.087(4)$ \\
$\mathrm{W} 1-\mathrm{O} 3$ & $1.859(3)$ & $\mathrm{Fe} 1-\mathrm{O} 76^{\mathrm{iii}}$ & $2.165(4)$ \\
$\mathrm{W} 1-\mathrm{O} 4$ & $1.979(3)$ & $\mathrm{Fe} 1-\mathrm{O} 76$ & $2.165(4)$ \\
$\mathrm{W} 1-\mathrm{O} 5$ & $2.273(3)$ & $\mathrm{Fe} 2-\mathrm{O} 19$ & $2.108(4)$ \\
$\mathrm{W} 1-\mathrm{O} 6$ & $1.930(4)$ & $\mathrm{Fe} 2-\mathrm{O} 26$ & $2.101(4)$
\end{tabular}




\begin{tabular}{|c|c|c|c|}
\hline $\mathrm{W} 2-\mathrm{O} 6$ & $1.882(3)$ & $\mathrm{Fe} 2-\mathrm{O} 27$ & $2.093(4)$ \\
\hline $\mathrm{W} 2-\mathrm{O} 7$ & $1.748(3)$ & $\mathrm{Fe} 2-\mathrm{O} 28$ & $2.169(4)$ \\
\hline $\mathrm{W} 2-\mathrm{O} 8$ & $2.259(4)$ & $\mathrm{Fe} 2-\mathrm{O} 29$ & $2.136(4)$ \\
\hline $\mathrm{W} 2-\mathrm{O} 9$ & $2.201(3)$ & $\mathrm{Fe} 2-\mathrm{O} 30$ & $2.122(4)$ \\
\hline $\mathrm{W} 2-\mathrm{O} 10$ & $1.754(4)$ & $\mathrm{Fe} 3-\mathrm{O} 14$ & $2.145(3)$ \\
\hline $\mathrm{W} 2-\mathrm{O} 11$ & $1.943(3)$ & $\mathrm{Fe} 3-\mathrm{O} 16$ & $2.087(4)$ \\
\hline $\mathrm{W} 3-\mathrm{O} 8$ & $2.195(3)$ & $\mathrm{Fe} 3-\mathrm{O} 22$ & $2.145(4)$ \\
\hline $\mathrm{W} 3-\mathrm{O} 11$ & $1.905(4)$ & $\mathrm{Fe} 3-\mathrm{O} 23$ & $2.116(4)$ \\
\hline $\mathrm{W} 3-\mathrm{O} 12$ & $1.743(3)$ & $\mathrm{Fe} 3-\mathrm{O} 24$ & $2.136(3)$ \\
\hline $\mathrm{W} 3-\mathrm{O} 13$ & $1.890(4)$ & $\mathrm{Fe} 3-\mathrm{O} 25$ & $2.106(4)$ \\
\hline $\mathrm{W} 3-\mathrm{O} 14$ & $1.786(3)$ & $\mathrm{Na} 1-\mathrm{O} 49$ & $2.434(4)$ \\
\hline W3-O17 & 2.297 (3) & $\mathrm{Na} 1-\mathrm{O} 49^{\mathrm{iv}}$ & $2.434(4)$ \\
\hline $\mathrm{W} 4-\mathrm{O} 4^{\mathrm{i}}$ & $1.869(3)$ & $\mathrm{Na} 1-\mathrm{O} 50$ & $2.507(4)$ \\
\hline $\mathrm{W} 4-\mathrm{O} 13$ & $1.980(3)$ & $\mathrm{Na} 1-\mathrm{O} 50^{\mathrm{iv}}$ & $2.507(4)$ \\
\hline $\mathrm{W} 4-\mathrm{O} 15$ & $1.748(4)$ & $\mathrm{Na} 1-\mathrm{O} 51^{\mathrm{iv}}$ & $2.402(4)$ \\
\hline W4-O16 & $1.782(3)$ & $\mathrm{Na} 1-\mathrm{O} 51$ & $2.402(4)$ \\
\hline W4-O17 & $2.226(4)$ & $\mathrm{Na} 2-\mathrm{Na}^{\mathrm{v}}$ & $4.194(3)$ \\
\hline $\mathrm{W} 4-\mathrm{O} 20^{\mathrm{i}}$ & $2.122(3)$ & $\mathrm{Na} 2-\mathrm{O} 76^{\mathrm{vi}}$ & $2.460(4)$ \\
\hline $\mathrm{W} 5-\mathrm{O} 2^{\mathrm{i}}$ & $2.071(4)$ & $\mathrm{Na} 2-\mathrm{O} 46$ & $2.394(4)$ \\
\hline $\mathrm{W} 5-\mathrm{O} 5^{\mathrm{i}}$ & $2.224(4)$ & $\mathrm{Na} 2-\mathrm{O} 47$ & $2.395(4)$ \\
\hline W5-O9 & $1.796(3)$ & $\mathrm{Na} 2-\mathrm{O} 48$ & $2.317(4)$ \\
\hline $\mathrm{W} 5-\mathrm{O} 17$ & $1.883(3)$ & $\mathrm{Na} 2-\mathrm{O} 49$ & $2.456(5)$ \\
\hline $\mathrm{W} 5-\mathrm{O} 18$ & $1.960(3)$ & $\mathrm{Na} 2-\mathrm{O} 52^{\mathrm{v}}$ & $2.606(4)$ \\
\hline W5-O19 & $1.743(4)$ & $\mathrm{Na} 3-\mathrm{Na} 2^{\mathrm{v}}$ & 4.194 (3) \\
\hline $\mathrm{W} 6-\mathrm{O}^{\mathrm{i}}$ & $2.043(3)$ & $\mathrm{Na} 3-\mathrm{O} 37$ & $2.484(4)$ \\
\hline $\mathrm{W} 6-\mathrm{O} 5^{\mathrm{i}}$ & $2.271(3)$ & $\mathrm{Na} 3-\mathrm{O} 52$ & $2.412(4)$ \\
\hline $\mathrm{W} 6-08^{\mathrm{i}}$ & $1.923(4)$ & $\mathrm{Na} 3-\mathrm{O} 53$ & $2.392(5)$ \\
\hline $\mathrm{W} 6-\mathrm{O} 18$ & $1.942(4)$ & $\mathrm{Na} 3-\mathrm{O} 54$ & $2.375(4)$ \\
\hline $\mathrm{W} 6-\mathrm{O} 20$ & $1.826(3)$ & $\mathrm{Na} 3-\mathrm{O} 55$ & $2.416(4)$ \\
\hline $\mathrm{W} 6-\mathrm{O} 21$ & $1.732(4)$ & $\mathrm{Na} 3-\mathrm{O} 56$ & $2.408(4)$ \\
\hline $\mathrm{W} 7-\mathrm{O} 75$ & $1.746(3)$ & $\mathrm{Na} 4-\mathrm{Na} 5$ & $3.996(3)$ \\
\hline $\mathrm{W} 7-\mathrm{O} 26$ & $1.781(4)$ & $\mathrm{Na} 4-\mathrm{O} 10^{\text {vii }}$ & $2.477(4)$ \\
\hline $\mathrm{W} 7-\mathrm{O} 31$ & $1.913(4)$ & $\mathrm{Na} 4-\mathrm{O} 58$ & $2.407(5)$ \\
\hline $\mathrm{W} 7-\mathrm{O} 32$ & $2.247(3)$ & $\mathrm{Na} 4-\mathrm{O} 59$ & $2.383(4)$ \\
\hline $\mathrm{W} 7-\mathrm{O} 33$ & $2.174(3)$ & $\mathrm{Na} 4-\mathrm{O} 60$ & $2.427(4)$ \\
\hline $\mathrm{W} 7-\mathrm{O} 35$ & $1.888(4)$ & $\mathrm{Na} 4-\mathrm{O} 62$ & $2.395(4)$ \\
\hline $\mathrm{W} 8-\mathrm{O} 33$ & $2.258(4)$ & $\mathrm{Na} 4-\mathrm{O} 67$ & $2.302(11)$ \\
\hline $\mathrm{W} 8-\mathrm{O} 34$ & $2.166(3)$ & $\mathrm{Na} 4-\mathrm{O} 167$ & $2.382(13)$ \\
\hline $\mathrm{W} 8-\mathrm{O} 35$ & $1.961(3)$ & $\mathrm{Na} 5-\mathrm{Na} 6$ & $4.167(2)$ \\
\hline $\mathrm{W} 8-\mathrm{O} 36$ & $1.759(3)$ & $\mathrm{Na} 5-\mathrm{O} 21$ & $2.447(4)$ \\
\hline $\mathrm{W} 8-\mathrm{O} 37$ & $1.750(4)$ & $\mathrm{Na} 5-\mathrm{O} 61$ & $2.380(4)$ \\
\hline $\mathrm{W} 8-\mathrm{O} 39^{\mathrm{ii}}$ & $1.860(3)$ & $\mathrm{Na} 5-\mathrm{O} 62$ & $2.418(4)$ \\
\hline W9-O25 & $1.743(4)$ & $\mathrm{Na} 5-\mathrm{O} 63$ & $2.416(4)$ \\
\hline W9-O32 & $1.895(4)$ & $\mathrm{Na} 5-\mathrm{O} 74$ & $2.424(4)$ \\
\hline W9-O34 & $1.797(4)$ & $\mathrm{Na} 5-\mathrm{O} 66$ & $2.379(11)$ \\
\hline W9-O42 & $2.213(4)$ & $\mathrm{Na} 5-\mathrm{O} 166$ & $2.412(12)$ \\
\hline W9-O43 & $2.079(4)$ & $\mathrm{Na} 6-\mathrm{Na} 5^{\text {viii }}$ & $4.167(2)$ \\
\hline W9-O45 & $1.943(3)$ & $\mathrm{Na} 6-\mathrm{O} 64^{\text {viii }}$ & $2.344(4)$ \\
\hline
\end{tabular}




\begin{tabular}{|c|c|c|c|}
\hline $\mathrm{W} 10-\mathrm{O} 33^{\mathrm{ii}}$ & $1.922(4)$ & $\mathrm{Na} 6-\mathrm{O} 64$ & $2.344(4)$ \\
\hline $\mathrm{W} 10-\mathrm{O} 41$ & $2.052(3)$ & $\mathrm{Na} 6-\mathrm{O} 65$ & $2.404(4)$ \\
\hline $\mathrm{W} 10-\mathrm{O} 42$ & $2.268(3)$ & $\mathrm{Na} 6-\mathrm{O} 65^{\text {viii }}$ & $2.404(4)$ \\
\hline $\mathrm{W} 10-\mathrm{O} 44^{\mathrm{ii}}$ & $1.835(3)$ & $\mathrm{Na} 6-\mathrm{O} 66$ & $2.358(11)$ \\
\hline $\mathrm{W} 10-\mathrm{O} 45$ & $1.947(4)$ & $\mathrm{Na} 6-\mathrm{O}_{6} 6^{\text {viii }}$ & $2.358(11)$ \\
\hline $\mathrm{W} 10-\mathrm{O} 46$ & $1.724(4)$ & $\mathrm{Na} 6-\mathrm{O} 166^{\text {viii }}$ & $2.606(11)$ \\
\hline $\mathrm{W} 11-\mathrm{O} 38$ & $1.942(3)$ & $\mathrm{Na} 6-\mathrm{O} 166$ & $2.606(11)$ \\
\hline $\mathrm{W} 11-\mathrm{O} 39$ & $1.958(4)$ & $\mathrm{O} 2-\mathrm{W} 5^{\mathrm{i}}$ & $2.071(3)$ \\
\hline $\mathrm{W} 11-\mathrm{O} 40$ & $1.719(4)$ & $\mathrm{O} 76-\mathrm{Na} 2^{\mathrm{ix}}$ & $2.460(4)$ \\
\hline $\mathrm{W} 11-\mathrm{O} 41$ & $1.892(3)$ & $\mathrm{O} 3-\mathrm{W} 6^{\mathrm{i}}$ & $2.043(3)$ \\
\hline W11-O42 & $2.283(3)$ & $\mathrm{O} 4-\mathrm{W} 4^{\mathrm{i}}$ & $1.869(3)$ \\
\hline $\mathrm{W} 11-\mathrm{O} 43$ & $1.870(4)$ & $\mathrm{O} 5-\mathrm{W} 5^{\mathrm{i}}$ & $2.224(3)$ \\
\hline $\mathrm{W} 12-\mathrm{O} 78$ & $1.743(4)$ & $\mathrm{O} 5-\mathrm{W} 6^{\mathrm{i}}$ & $2.271(3)$ \\
\hline $\mathrm{W} 12-\mathrm{O} 30$ & $1.775(4)$ & $\mathrm{O} 8-\mathrm{W} 6^{\mathrm{i}}$ & $1.923(4)$ \\
\hline $\mathrm{W} 12-\mathrm{O} 31$ & $1.960(3)$ & $\mathrm{O} 10-\mathrm{Na} 4^{\mathrm{vii}}$ & $2.477(4)$ \\
\hline $\mathrm{W} 12-\mathrm{O} 32$ & $2.237(4)$ & $\mathrm{O} 20-\mathrm{W} 4^{\mathrm{i}}$ & $2.122(3)$ \\
\hline $\mathrm{W} 12-\mathrm{O} 38$ & $1.879(3)$ & $\mathrm{O} 33-\mathrm{W} 10^{\mathrm{ii}}$ & $1.922(4)$ \\
\hline $\mathrm{W} 12-\mathrm{O} 44$ & $2.146(3)$ & $\mathrm{O} 39-\mathrm{W} 8^{\mathrm{ii}}$ & $1.860(3)$ \\
\hline $\mathrm{Fe} 1-\mathrm{O} 75^{\mathrm{iii}}$ & $2.100(3)$ & $\mathrm{O} 44-\mathrm{W} 10^{\mathrm{ii}}$ & $1.835(3)$ \\
\hline $\mathrm{Fe} 1-\mathrm{O} 75$ & $2.100(3)$ & $\mathrm{O} 52-\mathrm{Na} 2^{\mathrm{v}}$ & $2.606(4)$ \\
\hline $\mathrm{O} 67 \mathrm{a}-\mathrm{Na} 4-\mathrm{Na} 5$ & $72.3(3)$ & $\mathrm{O} 38-\mathrm{W} 12-\mathrm{O} 44$ & $86.63(14)$ \\
\hline $\mathrm{O} 167 \mathrm{~b}-\mathrm{Na} 4-\mathrm{Na} 5$ & $91.8(3)$ & $\mathrm{O} 44-\mathrm{W} 12-\mathrm{O} 32$ & $76.83(13)$ \\
\hline $\mathrm{O} 67 \mathrm{a}-\mathrm{Na} 4-\mathrm{O} 10^{\text {vii }}$ & $91.4(3)$ & $\mathrm{O} 75^{\mathrm{iii}}-\mathrm{Fe} 1-\mathrm{O} 75$ & $180.00(17)$ \\
\hline $\mathrm{O} 1-\mathrm{W} 1-\mathrm{O} 2$ & $102.23(16)$ & $\mathrm{O} 75-\mathrm{Fe} 1-\mathrm{O} 76$ & $84.52(14)$ \\
\hline $\mathrm{O} 1-\mathrm{W} 1-\mathrm{O} 3$ & $102.82(16)$ & $\mathrm{O} 75^{\mathrm{iii}}-\mathrm{Fe} 1-\mathrm{O} 76^{\mathrm{iii}}$ & $84.51(14)$ \\
\hline $\mathrm{O} 1-\mathrm{W} 1-\mathrm{O} 4$ & $100.36(16)$ & $\mathrm{O} 75-\mathrm{Fe} 1-\mathrm{O} 76^{\mathrm{iii}}$ & $95.48(14)$ \\
\hline $\mathrm{O} 1-\mathrm{W} 1-\mathrm{O} 5$ & $177.56(16)$ & $\mathrm{O} 75^{\mathrm{iii}}-\mathrm{Fe} 1-\mathrm{O} 76$ & $95.49(14)$ \\
\hline $\mathrm{O} 1-\mathrm{W} 1-\mathrm{O} 6$ & $102.08(16)$ & $\mathrm{O} 77-\mathrm{Fe} 1-\mathrm{O} 75$ & $88.54(15)$ \\
\hline $\mathrm{O} 167 \mathrm{~b}-\mathrm{Na} 4-\mathrm{O} 10^{\mathrm{vii}}$ & $92.8(3)$ & $\mathrm{O} 77^{\mathrm{iii}}-\mathrm{Fe} 1-\mathrm{O} 75^{\mathrm{iii}}$ & $88.54(15)$ \\
\hline $\mathrm{O} 167 \mathrm{~b}-\mathrm{Na} 4-\mathrm{O} 58$ & $160.3(3)$ & $\mathrm{O} 77^{\mathrm{iii}}-\mathrm{Fe} 1-\mathrm{O} 75$ & $91.46(15)$ \\
\hline $\mathrm{O} 67 \mathrm{a}-\mathrm{Na} 4-\mathrm{O} 58$ & $164.0(3)$ & $\mathrm{O} 77-\mathrm{Fe} 1-\mathrm{O} 75^{\mathrm{iii}}$ & $91.46(15)$ \\
\hline $\mathrm{O} 167 \mathrm{~b}-\mathrm{Na} 4-\mathrm{O} 59$ & $80.4(3)$ & $\mathrm{O} 77^{\mathrm{iii}}-\mathrm{Fe} 1-\mathrm{O} 77$ & $180.0(2)$ \\
\hline $\mathrm{O} 67 \mathrm{a}-\mathrm{Na} 4-\mathrm{O} 59$ & $82.0(3)$ & $\mathrm{O} 77-\mathrm{Fe} 1-\mathrm{O}^{6} 6^{\mathrm{iii}}$ & $89.27(15)$ \\
\hline $\mathrm{O} 67 \mathrm{a}-\mathrm{Na} 4-\mathrm{O} 60$ & $100.9(3)$ & $\mathrm{O} 77^{\mathrm{iii}}-\mathrm{Fe} 1-\mathrm{O} 76^{\mathrm{iii}}$ & $90.73(15)$ \\
\hline $\mathrm{O} 167 \mathrm{~b}-\mathrm{Na} 4-\mathrm{O} 60$ & $79.8(3)$ & $\mathrm{O} 77^{\mathrm{iii}}-\mathrm{Fe} 1-\mathrm{O} 76$ & $89.27(15)$ \\
\hline $\mathrm{O} 67 \mathrm{a}-\mathrm{Na} 4-\mathrm{O} 62$ & $91.2(3)$ & $\mathrm{O} 77-\mathrm{Fe} 1-\mathrm{O} 76$ & $90.73(15)$ \\
\hline $\mathrm{O} 167 \mathrm{~b}-\mathrm{Na} 4-\mathrm{O} 62$ & $112.6(3)$ & $\mathrm{O} 76^{\mathrm{iii}}-\mathrm{Fe} 1-\mathrm{O} 76$ & 180.0 \\
\hline $\mathrm{O} 66 \mathrm{a}-\mathrm{Na} 5-\mathrm{Na} 4$ & $91.9(2)$ & $\mathrm{O} 19-\mathrm{Fe} 2-\mathrm{O} 28$ & $94.86(14)$ \\
\hline $\mathrm{O} 2-\mathrm{W} 1-\mathrm{O} 4$ & $86.64(15)$ & $\mathrm{O} 19-\mathrm{Fe} 2-\mathrm{O} 29$ & $88.73(14)$ \\
\hline $\mathrm{O} 2-\mathrm{W} 1-\mathrm{O} 5$ & $75.66(14)$ & $\mathrm{O} 19-\mathrm{Fe} 2-\mathrm{O} 30$ & $172.37(14)$ \\
\hline $\mathrm{O} 2-\mathrm{W} 1-\mathrm{O} 6$ & $154.94(15)$ & $\mathrm{O} 26-\mathrm{Fe} 2-\mathrm{O} 19$ & $86.58(14)$ \\
\hline $\mathrm{O} 166 \mathrm{~b}-\mathrm{Na} 5-\mathrm{Na} 4$ & $74.3(3)$ & $\mathrm{O} 26-\mathrm{Fe} 2-\mathrm{O} 28$ & $84.95(14)$ \\
\hline $\mathrm{O} 66 \mathrm{a}-\mathrm{Na} 5-\mathrm{Na} 6$ & $28.3(3)$ & $\mathrm{O} 26-\mathrm{Fe} 2-\mathrm{O} 29$ & $99.31(14)$ \\
\hline $\mathrm{O} 166 \mathrm{~b}-\mathrm{Na} 5-\mathrm{Na} 6$ & $35.4(3)$ & $\mathrm{O} 26-\mathrm{Fe} 2-\mathrm{O} 30$ & $93.00(14)$ \\
\hline $\mathrm{O} 166 \mathrm{~b}-\mathrm{Na} 5-\mathrm{O} 21$ & $163.0(3)$ & $\mathrm{O} 27-\mathrm{Fe} 2-\mathrm{O} 19$ & $88.57(15)$ \\
\hline $\mathrm{O} 3-\mathrm{W} 1-\mathrm{O} 2$ & $92.10(16)$ & $\mathrm{O} 27-\mathrm{Fe} 2-\mathrm{O} 26$ & $170.10(14)$ \\
\hline $\mathrm{O} 3-\mathrm{W} 1-\mathrm{O} 4$ & $156.51(14)$ & $\mathrm{O} 27-\mathrm{Fe} 2-\mathrm{O} 28$ & $86.87(14)$ \\
\hline
\end{tabular}




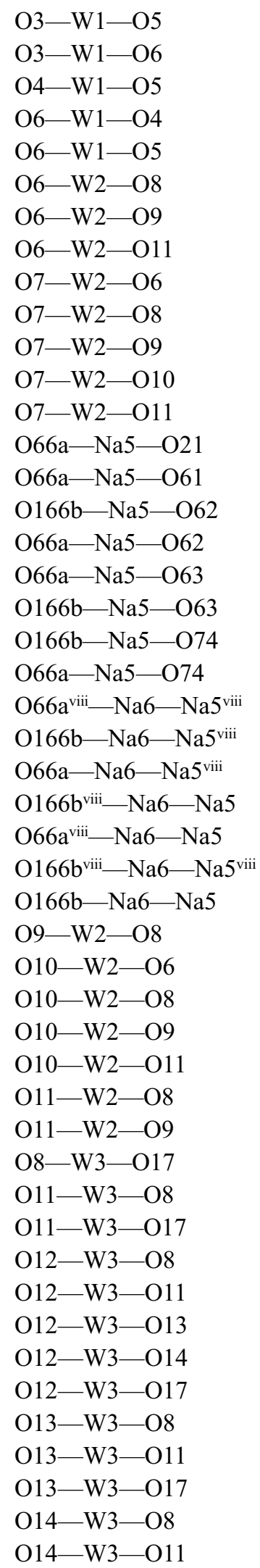

\begin{tabular}{|c|c|}
\hline $76.16(13)$ & $\mathrm{O} 27-\mathrm{Fe} 2-\mathrm{O} 29$ \\
\hline $88.40(15)$ & $\mathrm{O} 27-\mathrm{Fe} 2-\mathrm{O} 30$ \\
\hline $80.82(13)$ & $\mathrm{O} 29-\mathrm{Fe} 2-\mathrm{O} 28$ \\
\hline $83.09(15)$ & $\mathrm{O} 30-\mathrm{Fe} 2-\mathrm{O} 28$ \\
\hline $80.15(14)$ & $\mathrm{O} 30-\mathrm{Fe} 2-\mathrm{O} 29$ \\
\hline $86.58(14)$ & $\mathrm{O} 16-\mathrm{Fe} 3-\mathrm{O} 14$ \\
\hline $82.77(14)$ & $\mathrm{O} 16-\mathrm{Fe} 3-\mathrm{O} 22$ \\
\hline $155.70(15)$ & $\mathrm{O} 16-\mathrm{Fe} 3-\mathrm{O} 23$ \\
\hline $97.75(16)$ & $\mathrm{O} 16-\mathrm{Fe} 3-\mathrm{O} 24$ \\
\hline 93.99 (16) & $\mathrm{O} 16-\mathrm{Fe} 3-\mathrm{O} 25$ \\
\hline $170.19(16)$ & $\mathrm{O} 22-\mathrm{Fe} 3-\mathrm{O} 14$ \\
\hline $102.74(17)$ & $\mathrm{O} 23-\mathrm{Fe} 3-\mathrm{O} 14$ \\
\hline $95.65(15)$ & $\mathrm{O} 23-\mathrm{Fe} 3-\mathrm{O} 22$ \\
\hline $176.3(3)$ & $\mathrm{O} 23-\mathrm{Fe} 3-\mathrm{O} 24$ \\
\hline $96.4(3)$ & $\mathrm{O} 24-\mathrm{Fe} 3-\mathrm{O} 14$ \\
\hline $71.7(3)$ & $\mathrm{O} 24-\mathrm{Fe} 3-\mathrm{O} 22$ \\
\hline $84.3(3)$ & $\mathrm{O} 25-\mathrm{Fe} 3-\mathrm{O} 14$ \\
\hline $105.1(3)$ & $\mathrm{O} 25-\mathrm{Fe} 3-\mathrm{O} 22$ \\
\hline $97.0(3)$ & $\mathrm{O} 25-\mathrm{Fe} 3-\mathrm{O} 23$ \\
\hline $88.6(3)$ & $\mathrm{O} 25-\mathrm{Fe} 3-\mathrm{O} 24$ \\
\hline $74.2(3)$ & $\mathrm{O} 49-\mathrm{Na} 1-\mathrm{O} 49^{\mathrm{iv}}$ \\
\hline $28.5(3)$ & $\mathrm{O} 49-\mathrm{Na} 1-\mathrm{O} 50^{\mathrm{iv}}$ \\
\hline $147.6(3)$ & $\mathrm{O} 49^{\mathrm{iv}}-\mathrm{Na} 1-\mathrm{O} 50^{\mathrm{iv}}$ \\
\hline $151.5(3)$ & $\mathrm{O} 49^{\mathrm{iv}}-\mathrm{Na} 1-\mathrm{O} 50$ \\
\hline $147.6(3)$ & $\mathrm{O} 49-\mathrm{Na} 1-\mathrm{O} 50$ \\
\hline $151.5(3)$ & $\mathrm{O} 50^{\mathrm{iv}}-\mathrm{Na} 1-\mathrm{O} 50$ \\
\hline $32.4(3)$ & $\mathrm{O} 51-\mathrm{Na} 1-\mathrm{O} 49^{\mathrm{iv}}$ \\
\hline $32.4(3)$ & $\mathrm{O} 51-\mathrm{Na} 1-\mathrm{O} 49$ \\
\hline $76.25(13)$ & $\mathrm{O} 51^{\mathrm{iv}}-\mathrm{Na} 1-\mathrm{O} 49$ \\
\hline 100.05 (16) & $\mathrm{O} 51^{\mathrm{iv}}-\mathrm{Na} 1-\mathrm{O} 49^{\mathrm{iv}}$ \\
\hline $160.90(15)$ & $\mathrm{O} 51^{\mathrm{iv}}-\mathrm{Na} 1-\mathrm{O} 50^{\mathrm{iv}}$ \\
\hline $86.76(15)$ & $\mathrm{O} 51-\mathrm{Na} 1-\mathrm{O} 50^{\mathrm{iv}}$ \\
\hline $96.62(16)$ & $\mathrm{O} 51^{\mathrm{iv}}-\mathrm{Na} 1-\mathrm{O} 50$ \\
\hline $72.31(14)$ & $\mathrm{O} 51-\mathrm{Na} 1-\mathrm{O} 50$ \\
\hline $80.65(13)$ & $\mathrm{O} 51-\mathrm{Na} 1-\mathrm{O} 51^{\mathrm{iv}}$ \\
\hline $77.47(13)$ & $\mathrm{O} 76^{\mathrm{vi}}-\mathrm{Na} 2-\mathrm{Na}^{\mathrm{v}}$ \\
\hline 74.48 (14) & $\mathrm{O} 76^{\mathrm{vi}}-\mathrm{Na} 2-\mathrm{O} 52^{\mathrm{v}}$ \\
\hline $85.82(14)$ & $\mathrm{O} 46-\mathrm{Na} 2-\mathrm{Na}^{\mathrm{v}}$ \\
\hline $94.71(15)$ & $\mathrm{O} 46-\mathrm{Na} 2-\mathrm{O} 76^{\mathrm{vi}}$ \\
\hline $100.68(16)$ & $\mathrm{O} 46-\mathrm{Na} 2-\mathrm{O} 47$ \\
\hline $97.09(16)$ & $\mathrm{O} 46-\mathrm{Na} 2-\mathrm{O} 49$ \\
\hline $103.75(16)$ & $\mathrm{O} 46-\mathrm{Na} 2-\mathrm{O} 52^{\mathrm{v}}$ \\
\hline $168.24(16)$ & $\mathrm{O} 47-\mathrm{Na} 2-\mathrm{Na}^{\mathrm{v}}$ \\
\hline $85.38(14)$ & $\mathrm{O} 47-\mathrm{Na} 2-\mathrm{O} 76^{\mathrm{vi}}$ \\
\hline $154.04(14)$ & $\mathrm{O} 47-\mathrm{Na} 2-\mathrm{O} 49$ \\
\hline $73.75(14)$ & $\mathrm{O} 47-\mathrm{Na} 2-\mathrm{O} 52^{\mathrm{v}}$ \\
\hline $160.44(14)$ & $\mathrm{O} 48-\mathrm{Na} 2-\mathrm{Na}^{\mathrm{v}}$ \\
\hline $95.49(16)$ & $\mathrm{O} 48-\mathrm{Na} 2-\mathrm{O} 76^{\mathrm{vi}}$ \\
\hline
\end{tabular}

89.20 (14)

92.94 (15)

174.61 (15) 92.69 (14) 83.82 (14) 93.57 (14) 92.74 (14) 94.67 (15) 83.16 (14) $171.65(14)$ 83.38 (14) 167.85 (14) 87.31 (14) 88.30 (14) 101.55 (14) $173.74(15)$ $87.26(14)$ 95.61 (14) 85.89 (14) 88.53 (14) 180.0 94.06 (13) 85.94 (13) 94.06 (13) 85.94 (13) 180.0 87.15 (13) 92.85 (13) 87.15 (13) 92.85 (13) 90.54 (14) 89.46 (14) 89.46 (14) 90.54 (14) 180.0 115.31 (11) 85.72 (14) 80.08 (10) 76.13 (14) 83.98 (14) 151.08 (16) 83.19 (14) $69.12(11)$ 158.13 (17) 92.49 (15) 101.02 (15) 144.46 (14) 97.84 (16) 


\begin{tabular}{|c|c|}
\hline $\mathrm{O} 14-\mathrm{W} 3-\mathrm{O} 13$ & $98.50(16)$ \\
\hline $\mathrm{O} 14-\mathrm{W} 3-\mathrm{O} 17$ & $85.19(14)$ \\
\hline $\mathrm{O} 4 \mathrm{i}-\mathrm{W} 4-\mathrm{O} 13$ & $158.70(15)$ \\
\hline $\mathrm{O} 4 \mathrm{i}-\mathrm{W} 4-\mathrm{O} 17$ & $87.50(14)$ \\
\hline $\mathrm{O} 4^{\mathrm{i}}-\mathrm{W} 4-\mathrm{O} 20^{\mathrm{i}}$ & $87.80(14)$ \\
\hline $\mathrm{O} 13-\mathrm{W} 4-\mathrm{O} 17$ & $73.81(14)$ \\
\hline $\mathrm{O} 13-\mathrm{W} 4-\mathrm{O} 20^{\mathrm{i}}$ & $78.33(13)$ \\
\hline $\mathrm{O} 15-\mathrm{W} 4-\mathrm{O} 4^{\mathrm{i}}$ & $99.78(16)$ \\
\hline $\mathrm{O} 15-\mathrm{W} 4-\mathrm{O} 13$ & $96.69(16)$ \\
\hline $\mathrm{O} 15-\mathrm{W} 4-\mathrm{O} 16$ & $102.35(17)$ \\
\hline $\mathrm{O} 15-\mathrm{W} 4-\mathrm{O} 17$ & $166.45(15)$ \\
\hline $\mathrm{O} 15-\mathrm{W} 4-\mathrm{O} 20^{\mathrm{i}}$ & $91.54(16)$ \\
\hline $\mathrm{O} 16-\mathrm{W} 4-\mathrm{O} 4^{\mathrm{i}}$ & $98.64(16)$ \\
\hline $\mathrm{O} 16-\mathrm{W} 4-\mathrm{O} 13$ & $90.86(15)$ \\
\hline $\mathrm{O} 16-\mathrm{W} 4-\mathrm{O} 17$ & $87.68(16)$ \\
\hline $\mathrm{O} 16-\mathrm{W} 4-\mathrm{O} 20^{\mathrm{i}}$ & $163.37(16)$ \\
\hline $\mathrm{O} 20^{\mathrm{i}}-\mathrm{W} 4-\mathrm{O} 17$ & $77.24(14)$ \\
\hline $\mathrm{O} 2^{\mathrm{i}}-\mathrm{W} 5-\mathrm{O} 5^{\mathrm{i}}$ & $73.17(13)$ \\
\hline $\mathrm{O} 9-\mathrm{W} 5-\mathrm{O} 2^{\mathrm{i}}$ & $161.79(16)$ \\
\hline $\mathrm{O} 9-\mathrm{W} 5-\mathrm{O} 5^{\mathrm{i}}$ & $88.63(15)$ \\
\hline $\mathrm{O} 9-\mathrm{W} 5-\mathrm{O} 17$ & $94.60(16)$ \\
\hline $\mathrm{O} 9-\mathrm{W} 5-\mathrm{O} 18$ & $92.85(15)$ \\
\hline $\mathrm{O} 17-\mathrm{W} 5-\mathrm{O} 2^{\mathrm{i}}$ & $83.63(14)$ \\
\hline $\mathrm{O} 17-\mathrm{W} 5-\mathrm{O}^{\mathrm{i}}$ & $86.14(15)$ \\
\hline $\mathrm{O} 66 \mathrm{a}-\mathrm{Na} 6-\mathrm{Na} 5$ & $28.5(3)$ \\
\hline $\mathrm{O} 66 \mathrm{a}-\mathrm{Na} 6-\mathrm{O} 65^{\mathrm{viii}}$ & $81.5(3)$ \\
\hline O66a ${ }^{\text {viii }-N a 6-O 65^{\text {viii }}}$ & $98.5(3)$ \\
\hline $\mathrm{O}^{2} \mathrm{a}^{\mathrm{vii}-\mathrm{Na} 6}-\mathrm{O} 65$ & $81.5(3)$ \\
\hline $\mathrm{O} 66 \mathrm{a}-\mathrm{Na} 6-\mathrm{O} 65$ & $98.5(3)$ \\
\hline O66a-Na6-O66 ${ }^{\text {viii }}$ & 180.0 \\
\hline $\mathrm{O} 166 \mathrm{~b}-\mathrm{Na} 6-\mathrm{O} 166^{\mathrm{viii}}$ & 180.0 \\
\hline $\mathrm{Na} 5-\mathrm{O} 166 \mathrm{~b}-\mathrm{Na} 6$ & $112.2(5)$ \\
\hline $\mathrm{Na} 6-\mathrm{O} 66 \mathrm{a}-\mathrm{Na} 5$ & $123.2(5)$ \\
\hline O17-W5-O18 & $156.21(16)$ \\
\hline $\mathrm{O} 18-\mathrm{W} 5-\mathrm{O} 2^{\mathrm{i}}$ & $82.29(14)$ \\
\hline $\mathrm{O} 18-\mathrm{W} 5-\mathrm{O} 5^{\mathrm{i}}$ & $71.47(14)$ \\
\hline $\mathrm{O} 19-\mathrm{W} 5-\mathrm{O} 2^{\mathrm{i}}$ & $94.39(15)$ \\
\hline $\mathrm{O} 19-\mathrm{W} 5-\mathrm{O}^{\mathrm{i}}$ & $163.66(14)$ \\
\hline O19-W5-O9 & $103.63(17)$ \\
\hline O19-W5-O17 & $103.30(16)$ \\
\hline O19-W5-O18 & $96.82(16)$ \\
\hline $\mathrm{O} 3^{\mathrm{i}}-\mathrm{W} 6-\mathrm{O}^{\mathrm{i}}$ & $72.91(13)$ \\
\hline $\mathrm{O} 8^{\mathrm{i}}-\mathrm{W} 6-\mathrm{O} 3^{\mathrm{i}}$ & $84.52(15)$ \\
\hline 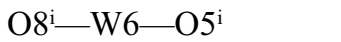 & $85.68(14)$ \\
\hline $\mathrm{O} 8 \mathrm{i}-\mathrm{W} 6-\mathrm{O} 18$ & $155.62(15)$ \\
\hline $\mathrm{O} 18-\mathrm{W} 6-\mathrm{O} 3^{\mathrm{i}}$ & $82.76(14)$ \\
\hline $\mathrm{O} 18-\mathrm{W} 6-\mathrm{O} 5^{\mathrm{i}}$ & $70.73(14)$ \\
\hline $\mathrm{O} 20-\mathrm{W} 6-\mathrm{O} 3^{\mathrm{i}}$ & $160.28(14)$ \\
\hline
\end{tabular}

\begin{tabular}{|c|c|}
\hline $\mathrm{O} 48-\mathrm{Na} 2-\mathrm{O} 46$ & $122.29(18)$ \\
\hline $\mathrm{O} 48-\mathrm{Na} 2-\mathrm{O} 47$ & $85.06(16)$ \\
\hline $\mathrm{O} 48-\mathrm{Na} 2-\mathrm{O} 49$ & $85.78(16)$ \\
\hline $\mathrm{O} 48-\mathrm{Na} 2-\mathrm{O} 52^{\mathrm{v}}$ & $154.45(18)$ \\
\hline $\mathrm{O} 49-\mathrm{Na} 2-\mathrm{Na}^{\mathrm{v}}$ & $71.91(11)$ \\
\hline $\mathrm{O} 49-\mathrm{Na} 2-\mathrm{O} 76^{\mathrm{vi}}$ & $109.31(15)$ \\
\hline $\mathrm{O} 49-\mathrm{Na} 2-\mathrm{O} 52^{\mathrm{v}}$ & $69.29(14)$ \\
\hline $\mathrm{O} 52^{\mathrm{v}}-\mathrm{Na} 2-\mathrm{Na}^{\mathrm{v}}$ & $31.89(9)$ \\
\hline $\mathrm{O} 37-\mathrm{Na} 3-\mathrm{Na} 2^{\mathrm{v}}$ & $116.60(11)$ \\
\hline $\mathrm{O} 52-\mathrm{Na} 3-\mathrm{Na}_{2}{ }^{\mathrm{v}}$ & $34.80(10)$ \\
\hline $\mathrm{O} 52-\mathrm{Na} 3-\mathrm{O} 37$ & $88.80(14)$ \\
\hline $\mathrm{O} 52-\mathrm{Na} 3-\mathrm{O} 55$ & $85.00(15)$ \\
\hline $\mathrm{O} 53-\mathrm{Na} 3-\mathrm{Na} 2^{v}$ & $66.75(11)$ \\
\hline $\mathrm{O} 53-\mathrm{Na} 3-\mathrm{O} 37$ & $86.52(14)$ \\
\hline $\mathrm{O} 53-\mathrm{Na} 3-\mathrm{O} 52$ & $83.44(15)$ \\
\hline $\mathrm{O} 53-\mathrm{Na} 3-\mathrm{O} 55$ & $165.38(17)$ \\
\hline $\mathrm{O} 53-\mathrm{Na} 3-\mathrm{O} 56$ & $104.30(16)$ \\
\hline $\mathrm{O} 54-\mathrm{Na} 3-\mathrm{Na}_{2}{ }^{\mathrm{v}}$ & $69.01(10)$ \\
\hline $\mathrm{O} 54-\mathrm{Na} 3-\mathrm{O} 37$ & $167.68(17)$ \\
\hline $\mathrm{O} 54-\mathrm{Na} 3-\mathrm{O} 52$ & $100.05(16)$ \\
\hline $\mathrm{O} 54-\mathrm{Na} 3-\mathrm{O} 53$ & $86.01(15)$ \\
\hline $\mathrm{O} 54-\mathrm{Na} 3-\mathrm{O} 55$ & $87.22(15)$ \\
\hline $\mathrm{O} 54-\mathrm{Na} 3-\mathrm{O} 56$ & $86.22(14)$ \\
\hline $\mathrm{O} 55-\mathrm{Na} 3-\mathrm{Na} 2^{\mathrm{v}}$ & $98.71(11)$ \\
\hline $\mathrm{O} 55-\mathrm{Na} 3-\mathrm{O} 37$ & $102.18(14)$ \\
\hline $\mathrm{O} 56-\mathrm{Na} 3-\mathrm{Na} 2^{\mathrm{v}}$ & $153.79(12)$ \\
\hline $\mathrm{O} 56-\mathrm{Na} 3-\mathrm{O} 37$ & $86.15(14)$ \\
\hline $\mathrm{O} 56-\mathrm{Na} 3-\mathrm{O} 52$ & $170.45(16)$ \\
\hline $\mathrm{O} 56-\mathrm{Na} 3-\mathrm{O} 55$ & $88.15(15)$ \\
\hline $\mathrm{O} 10^{\mathrm{vii}}-\mathrm{Na} 4-\mathrm{Na} 5$ & $110.91(11)$ \\
\hline $\mathrm{O} 58-\mathrm{Na} 4-\mathrm{Na} 5$ & $94.56(12)$ \\
\hline $\mathrm{O} 58-\mathrm{Na} 4-\mathrm{O} 10^{\mathrm{vii}}$ & $102.18(15)$ \\
\hline $\mathrm{O} 58-\mathrm{Na} 4-\mathrm{O} 60$ & $89.00(15)$ \\
\hline $\mathrm{O} 59-\mathrm{Na} 4-\mathrm{Na} 5$ & $68.57(10)$ \\
\hline $\mathrm{O} 59-\mathrm{Na} 4-\mathrm{O} 10^{\mathrm{vii}}$ & $173.18(17)$ \\
\hline $\mathrm{O} 59-\mathrm{Na} 4-\mathrm{O} 58$ & $84.62(15)$ \\
\hline $\mathrm{O} 59-\mathrm{Na} 4-\mathrm{O} 60$ & $95.17(15)$ \\
\hline $\mathrm{O} 59-\mathrm{Na} 4-\mathrm{O} 62$ & $98.88(15)$ \\
\hline $\mathrm{O} 60-\mathrm{Na} 4-\mathrm{Na} 5$ & $162.87(13)$ \\
\hline $\mathrm{O} 60-\mathrm{Na} 4-\mathrm{O} 10^{\mathrm{vii}}$ & $84.57(14)$ \\
\hline $\mathrm{O} 62-\mathrm{Na} 4-\mathrm{Na} 5$ & $34.07(10)$ \\
\hline $\mathrm{O} 62-\mathrm{Na} 4-\mathrm{O} 10^{\mathrm{vii}}$ & $82.69(14)$ \\
\hline $\mathrm{O} 62-\mathrm{Na} 4-\mathrm{O} 58$ & $82.18(15)$ \\
\hline $\mathrm{O} 62-\mathrm{Na} 4-\mathrm{O} 60$ & $162.60(16)$ \\
\hline $\mathrm{Na} 4-\mathrm{Na} 5-\mathrm{Na} 6$ & $90.45(5)$ \\
\hline $\mathrm{O} 21-\mathrm{Na} 5-\mathrm{Na} 4$ & $88.71(11)$ \\
\hline $\mathrm{O} 21-\mathrm{Na} 5-\mathrm{Na} 6$ & $148.08(11)$ \\
\hline $\mathrm{O} 61-\mathrm{Na} 5-\mathrm{Na} 4$ & $122.47(12)$ \\
\hline
\end{tabular}




$$
\begin{aligned}
& \mathrm{O} 20-\mathrm{W} 6-\mathrm{O} 5^{\mathrm{i}} \\
& \mathrm{O} 20-\mathrm{W} 6-\mathrm{O}^{\mathrm{i}} \\
& \mathrm{O} 20-\mathrm{W} 6-\mathrm{O} 18 \\
& \mathrm{O} 21-\mathrm{W} 6-\mathrm{O}^{\mathrm{i}} \\
& \mathrm{O} 21-\mathrm{W} 6-\mathrm{O}^{\mathrm{i}} \\
& \mathrm{O} 21-\mathrm{W} 6-\mathrm{O}^{\mathrm{i}} \\
& \text { O21-W6-O18 } \\
& \text { O21-W6-O20 } \\
& \text { O75-W7-O26 } \\
& \mathrm{O} 75-\mathrm{W} 7-\mathrm{O} 31 \\
& \mathrm{O} 75-\mathrm{W} 7-\mathrm{O} 32 \\
& \text { O75-W7-O33 } \\
& \text { O75-W7-O35 } \\
& \mathrm{O} 26-\mathrm{W} 7-\mathrm{O} 31 \\
& \mathrm{O} 26-\mathrm{W} 7-\mathrm{O} 32 \\
& \mathrm{O} 26-\mathrm{W} 7-\mathrm{O} 33 \\
& \mathrm{O} 26-\mathrm{W} 7-\mathrm{O} 35 \\
& \text { O31-W7-O32 } \\
& \text { O31-W7-O33 } \\
& \mathrm{O} 33-\mathrm{W} 7-\mathrm{O} 32 \\
& \mathrm{O} 35-\mathrm{W} 7-\mathrm{O} 31 \\
& \mathrm{O} 35-\mathrm{W} 7-\mathrm{O} 32 \\
& \mathrm{O} 35-\mathrm{W} 7-\mathrm{O} 33 \\
& \mathrm{O} 34-\mathrm{W} 8-\mathrm{O} 33 \\
& \mathrm{O} 35-\mathrm{W} 8-\mathrm{O} 33 \\
& \mathrm{O} 35-\mathrm{W} 8-\mathrm{O} 34 \\
& \text { O36-W8-O33 } \\
& \text { O36-W8-O34 } \\
& \mathrm{O} 36-\mathrm{W} 8-\mathrm{O} 35 \\
& \text { O36-W8-O39ii } \\
& \text { O37-W8-O33 } \\
& \text { O37-W8-O34 } \\
& \text { O37-W8-O35 } \\
& \text { O37-W8-O36 } \\
& \text { O37-W8-O39ii } \\
& \mathrm{O} 39^{\mathrm{ii}}-\mathrm{W} 8-\mathrm{O} 33 \\
& \mathrm{O} 39^{\mathrm{ii}}-\mathrm{W} 8-\mathrm{O} 34 \\
& \text { O39i-W8-O35 } \\
& \text { O25-W9-O32 } \\
& \text { O25-W9-O34 } \\
& \text { O25-W9-O42 } \\
& \text { O25-W9-O43 } \\
& \text { O25-W9-O45 } \\
& \text { O32-W9-O42 } \\
& \text { O32-W9-O43 } \\
& \text { O32-W9-O45 } \\
& \text { O34-W9-O32 } \\
& \text { O34-W9-O42 }
\end{aligned}
$$

$87.38(14)$
$94.35(16)$
$90.72(15)$
$96.16(15)$
$165.95(15)$
$102.36(16)$
$99.63(16)$
$103.29(16)$
$103.42(16)$
$94.64(16)$
$167.01(16)$
$93.94(15)$
$100.95(16)$
$98.53(16)$
$85.41(14)$
$161.89(14)$
$95.91(16)$
$74.41(14)$
$84.98(14)$
$78.40(13)$
$155.66(14)$
$87.39(14)$
$75.47(14)$
$76.31(13)$
$72.16(14)$
$79.27(14)$
$92.20(15)$
$167.70(15)$
$93.19(15)$
$97.99(15)$
$162.25(15)$
$88.05(15)$
$97.00(16)$
$102.61(17)$
$101.14(16)$
$86.20(15)$
$85.75(14)$
$156.00(15)$
$103.17(16)$
$104.38(17)$
$163.37(14)$
$92.49(15)$
$97.59(16)$
$84.68(14)$
$84.03(14)$
$155.61(16)$
$94.46(15)$
$89.37(15)$

$\mathrm{O} 61-\mathrm{Na} 5-\mathrm{Na} 6$

$\mathrm{O} 61-\mathrm{Na} 5-\mathrm{O} 21$

O61-Na5-O62

O61- Na5-O63

O61-Na5-O74

O61-Na5-O166

O62- $\mathrm{Na} 5-\mathrm{Na} 4$

$\mathrm{O} 62-\mathrm{Na} 5-\mathrm{Na} 6$

$\mathrm{O} 62-\mathrm{Na} 5-\mathrm{O} 21$

$\mathrm{O} 62-\mathrm{Na} 5-\mathrm{O} 74$

$\mathrm{O} 63-\mathrm{Na} 5-\mathrm{Na} 4$

$\mathrm{O} 63-\mathrm{Na} 5-\mathrm{Na} 6$

$\mathrm{O} 63-\mathrm{Na} 5-\mathrm{O} 21$

$\mathrm{O} 63-\mathrm{Na} 5-\mathrm{O} 62$

$\mathrm{O} 63-\mathrm{Na} 5-\mathrm{O} 74$

$\mathrm{O} 74-\mathrm{Na} 5-\mathrm{Na} 4$

$\mathrm{O} 74-\mathrm{Na} 5-\mathrm{Na} 6$

$\mathrm{O} 74-\mathrm{Na} 5-\mathrm{O} 21$

$\mathrm{Na} 5^{\text {viii- }} \mathrm{Na} 6-\mathrm{Na} 5$

$\mathrm{O} 64-\mathrm{Na} 6-\mathrm{Na} 5$

$\mathrm{O} 64^{\text {viii- }} \mathrm{Na}$ 6- $\mathrm{Na} 5^{\text {vii }}$

$\mathrm{O} 64-\mathrm{Na} 6-\mathrm{Na} 5^{\text {viii }}$

$\mathrm{O} 64^{\mathrm{vii}}-\mathrm{Na} 6-\mathrm{Na} 5$

$\mathrm{O} 64-\mathrm{Na} 6-\mathrm{O} 64^{\text {viii }}$

$\mathrm{O} 64-\mathrm{Na} 6-\mathrm{O} 65^{\text {viii }}$

$\mathrm{O} 64-\mathrm{Na} 6-\mathrm{O} 65$

O64 viii-Na6-O65

O64 ${ }^{\text {viii }-\mathrm{Na} 6-\mathrm{O} 65^{\text {viii }}}$

O64 viii_-Na6-O66

O64-Na6-O66

O64-Na6-O66 viii

O64 ${ }^{\text {viii-Na6-O66 }} 6^{\text {viii }}$

O64-Na6-O166 ${ }^{\text {viii }}$

O64-Na6-O166

O64 $4^{\text {viii-Na6-O166 }}{ }^{\text {viii }}$

O64 ${ }^{\mathrm{vii}}-\mathrm{Na} 6-\mathrm{O} 166$

O65-Na6-Na5

$\mathrm{O} 65^{\mathrm{viii}}-\mathrm{Na} 6-\mathrm{Na} 5^{\mathrm{viii}}$

O65-Na6-Na5 ${ }^{\text {viii }}$

O65 viii- $\mathrm{Na} 6-\mathrm{Na} 5$

O65-Na6-O65 viii

O65-Na6-O166 viii

O65 $5^{\text {viii }-\mathrm{Na} 6-\mathrm{O} 166^{\text {viii }}}$

O65 $5^{\mathrm{vii}}-\mathrm{Na} 6-\mathrm{O} 166$

O65-Na6-O166

$\mathrm{W} 7-\mathrm{O} 75-\mathrm{Fe} 1$

$\mathrm{W} 1-\mathrm{O} 2-\mathrm{W} 5^{\mathrm{i}}$

$\mathrm{Fe} 1-\mathrm{O} 76-\mathrm{Na} 2^{\mathrm{ix}}$
$73.42(11)$

80.23 (14)

90.73 (15)

$151.18(18)$

85.58 (15)

$108.6(3)$

33.69 (10)

68.87 (11)

$94.18(15)$

$157.62(18)$

76.45 (11)

$131.93(13)$

78.65 (14)

$110.12(15)$

82.05 (15)

$150.39(14)$

88.95 (13)

$106.88(17)$

180.0

$106.43(10)$

$106.43(11)$

73.57 (10)

73.57 (11)

180.0

89.83 (14)

$90.17(15)$

89.83 (14)

$90.17(15)$

96.6 (3)

83.4 (3)

$96.6(3)$

83.4 (3)

$105.8(3)$

74.2 (3)

$74.2(3)$

105.8 (3)

81.74 (11)

81.74 (11)

98.26 (11)

98.26 (11)

180.0

95.1 (3)

84.9 (3)

95.1 (3)

84.9 (3)

141.2 (2)

$115.40(17)$

123.52 (16) 


\begin{tabular}{|c|c|}
\hline $\mathrm{O} 34-\mathrm{W} 9-\mathrm{O} 43$ & $162.93(16)$ \\
\hline $\mathrm{O} 34-\mathrm{W} 9-\mathrm{O} 45$ & $92.62(15)$ \\
\hline $\mathrm{O} 43-\mathrm{W} 9-\mathrm{O} 42$ & $73.56(13)$ \\
\hline O45-W9-O42 & $72.07(14)$ \\
\hline $\mathrm{O} 45-\mathrm{W} 9-\mathrm{O} 43$ & $82.42(14)$ \\
\hline $\mathrm{O} 33^{\mathrm{ii}}-\mathrm{W} 10-\mathrm{O} 41$ & 84.49 (14) \\
\hline $\mathrm{O} 33^{\mathrm{ii}}-\mathrm{W} 10-\mathrm{O} 42$ & 85.99 (14) \\
\hline $\mathrm{O} 33^{\mathrm{ii}}-\mathrm{W} 10-\mathrm{O} 45$ & $155.71(15)$ \\
\hline $\mathrm{O} 41-\mathrm{W} 10-\mathrm{O} 42$ & $72.95(13)$ \\
\hline $\mathrm{O} 44^{\mathrm{ii}}-\mathrm{W} 10-\mathrm{O} 33^{\mathrm{ii}}$ & $94.56(15)$ \\
\hline 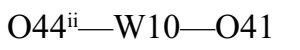 & $160.62(14)$ \\
\hline $\mathrm{O} 44^{\mathrm{ii}}-\mathrm{W} 10-\mathrm{O} 42$ & $87.67(14)$ \\
\hline $\mathrm{O} 44^{\mathrm{ii}}-\mathrm{W} 10-\mathrm{O} 45$ & $91.49(15)$ \\
\hline $\mathrm{O} 45-\mathrm{W} 10-\mathrm{O} 41$ & $82.10(14)$ \\
\hline $\mathrm{O} 45-\mathrm{W} 10-\mathrm{O} 42$ & $70.76(13)$ \\
\hline $\mathrm{O} 46-\mathrm{W} 10-\mathrm{O} 33^{\mathrm{ii}}$ & $102.68(16)$ \\
\hline $\mathrm{O} 46-\mathrm{W} 10-\mathrm{O} 41$ & $95.21(15)$ \\
\hline $\mathrm{O} 46-\mathrm{W} 10-\mathrm{O} 42$ & $164.79(15)$ \\
\hline $\mathrm{O} 46-\mathrm{W} 10-\mathrm{O} 44^{\mathrm{ii}}$ & $103.85(16)$ \\
\hline $\mathrm{O} 46-\mathrm{W} 10-\mathrm{O} 45$ & $98.64(16)$ \\
\hline $\mathrm{O} 38-\mathrm{W} 11-\mathrm{O} 39$ & $84.45(15)$ \\
\hline $\mathrm{O} 38-\mathrm{W} 11-\mathrm{O} 42$ & $81.24(14)$ \\
\hline $\mathrm{O} 39-\mathrm{W} 11-\mathrm{O} 42$ & $79.88(14)$ \\
\hline $\mathrm{O} 40-\mathrm{W} 11-\mathrm{O} 38$ & $102.46(16)$ \\
\hline $\mathrm{O} 40-\mathrm{W} 11-\mathrm{O} 39$ & $101.77(16)$ \\
\hline $\mathrm{O} 40-\mathrm{W} 11-\mathrm{O} 41$ & $100.97(16)$ \\
\hline $\mathrm{O} 40-\mathrm{W} 11-\mathrm{O} 42$ & $176.04(16)$ \\
\hline $\mathrm{O} 40-\mathrm{W} 11-\mathrm{O} 43$ & $102.78(17)$ \\
\hline $\mathrm{O} 41-\mathrm{W} 11-\mathrm{O} 38$ & $156.18(15)$ \\
\hline $\mathrm{O} 41-\mathrm{W} 11-\mathrm{O} 39$ & $86.64(15)$ \\
\hline $\mathrm{O} 41-\mathrm{W} 11-\mathrm{O} 42$ & $75.46(13)$ \\
\hline $\mathrm{O} 43-\mathrm{W} 11-\mathrm{O} 38$ & $87.92(15)$ \\
\hline $\mathrm{O} 43-\mathrm{W} 11-\mathrm{O} 39$ & $155.33(15)$ \\
\hline $\mathrm{O} 43-\mathrm{W} 11-\mathrm{O} 41$ & $91.09(15)$ \\
\hline $\mathrm{O} 43-\mathrm{W} 11-\mathrm{O} 42$ & $75.78(14)$ \\
\hline $\mathrm{O} 78-\mathrm{W} 12-\mathrm{O} 30$ & $102.32(17)$ \\
\hline $\mathrm{O} 78-\mathrm{W} 12-\mathrm{O} 31$ & $97.07(15)$ \\
\hline $\mathrm{O} 78-\mathrm{W} 12-\mathrm{O} 32$ & $166.24(15)$ \\
\hline $\mathrm{O} 78-\mathrm{W} 12-\mathrm{O} 38$ & $99.83(16)$ \\
\hline $\mathrm{O} 78-\mathrm{W} 12-\mathrm{O} 44$ & $91.39(15)$ \\
\hline $\mathrm{O} 30-\mathrm{W} 12-\mathrm{O} 31$ & $91.54(15)$ \\
\hline $\mathrm{O} 30-\mathrm{W} 12-\mathrm{O} 32$ & $88.42(16)$ \\
\hline $\mathrm{O} 30-\mathrm{W} 12-\mathrm{O} 38$ & $99.13(16)$ \\
\hline $\mathrm{O} 30-\mathrm{W} 12-\mathrm{O} 44$ & $163.89(16)$ \\
\hline $\mathrm{O} 31-\mathrm{W} 12-\mathrm{O} 32$ & $73.79(13)$ \\
\hline $\mathrm{O} 31-\mathrm{W} 12-\mathrm{O} 44$ & $78.25(13)$ \\
\hline
\end{tabular}

\begin{tabular}{|c|c|}
\hline $\mathrm{W} 1-\mathrm{O} 3-\mathrm{W} 6^{\mathrm{i}}$ & $116.84(17)$ \\
\hline $\mathrm{W} 4 \mathrm{i}-\mathrm{O} 4-\mathrm{W} 1$ & $147.3(2)$ \\
\hline $\mathrm{W} 5^{\mathrm{i}}-\mathrm{O} 5-\mathrm{W} 1$ & $95.76(13)$ \\
\hline $\mathrm{W} 5^{\mathrm{i}}-\mathrm{O} 5-\mathrm{W} 6^{\mathrm{i}}$ & $97.18(14)$ \\
\hline $\mathrm{W} 6 \mathrm{i}-\mathrm{O} 5-\mathrm{W} 1$ & $94.06(12)$ \\
\hline $\mathrm{W} 2-\mathrm{O} 6-\mathrm{W} 1$ & $149.1(2)$ \\
\hline $\mathrm{W} 3-\mathrm{O} 8-\mathrm{W} 2$ & $95.05(14)$ \\
\hline $\mathrm{W} 6{ }^{\mathrm{i}}-\mathrm{O} 8-\mathrm{W} 2$ & $137.59(17)$ \\
\hline $\mathrm{W} 6-\mathrm{O} 8-\mathrm{W} 3$ & $125.95(18)$ \\
\hline $\mathrm{W} 5-\mathrm{O} 9-\mathrm{W} 2$ & $137.20(18)$ \\
\hline $\mathrm{W} 2-\mathrm{O} 10-\mathrm{Na} 4^{\mathrm{vii}}$ & $123.67(18)$ \\
\hline W3-O11-W2 & $117.26(18)$ \\
\hline $\mathrm{W} 3-\mathrm{O} 13-\mathrm{W} 4$ & $117.31(18)$ \\
\hline $\mathrm{W} 3-\mathrm{O} 14-\mathrm{Fe} 3$ & $133.7(2)$ \\
\hline $\mathrm{W} 4-\mathrm{O} 16-\mathrm{Fe} 3$ & $138.8(2)$ \\
\hline $\mathrm{W} 4-\mathrm{O} 17-\mathrm{W} 3$ & $93.90(13)$ \\
\hline $\mathrm{W} 5-\mathrm{O} 17-\mathrm{W} 3$ & $125.71(18)$ \\
\hline $\mathrm{W} 5-\mathrm{O} 17-\mathrm{W} 4$ & $139.11(18)$ \\
\hline $\mathrm{W} 6-\mathrm{O} 18-\mathrm{W} 5$ & $119.54(18)$ \\
\hline W5-O19-Fe2 & $172.3(2)$ \\
\hline $\mathrm{W} 6-\mathrm{O} 20-\mathrm{W} 4^{\mathrm{i}}$ & $138.94(18)$ \\
\hline $\mathrm{W} 6-\mathrm{O} 21-\mathrm{Na} 5$ & $154.0(2)$ \\
\hline $\mathrm{W} 9-\mathrm{O} 25-\mathrm{Fe} 3$ & $169.9(2)$ \\
\hline $\mathrm{W} 7-\mathrm{O} 26-\mathrm{Fe} 2$ & $135.4(2)$ \\
\hline $\mathrm{W} 12-\mathrm{O} 30-\mathrm{Fe} 2$ & $137.9(2)$ \\
\hline $\mathrm{W} 7-\mathrm{O} 31-\mathrm{W} 12$ & $116.06(17)$ \\
\hline W9-O32-W7 & $124.93(18)$ \\
\hline W9-O32-W12 & $139.49(18)$ \\
\hline $\mathrm{W} 12-\mathrm{O} 32-\mathrm{W} 7$ & $94.25(13)$ \\
\hline $\mathrm{W} 7-\mathrm{O} 33-\mathrm{W} 8$ & $95.16(14)$ \\
\hline $\mathrm{W} 10^{\mathrm{ii}}-\mathrm{O} 33-\mathrm{W} 7$ & $125.80(18)$ \\
\hline $\mathrm{W} 10^{\mathrm{ii}}-\mathrm{O} 33-\mathrm{W} 8$ & $137.86(18)$ \\
\hline W9-O34-W8 & $139.68(18)$ \\
\hline $\mathrm{W} 7-\mathrm{O} 35-\mathrm{W} 8$ & $116.42(18)$ \\
\hline $\mathrm{W} 8-\mathrm{O} 37-\mathrm{Na} 3$ & $127.58(19)$ \\
\hline $\mathrm{W} 12-\mathrm{O} 38-\mathrm{W} 11$ & $147.9(2)$ \\
\hline $\mathrm{W} 8^{\mathrm{ii}}-\mathrm{O} 39-\mathrm{W} 11$ & $150.9(2)$ \\
\hline $\mathrm{W} 11-\mathrm{O} 41-\mathrm{W} 10$ & $116.54(16)$ \\
\hline $\mathrm{W} 9-\mathrm{O} 42-\mathrm{W} 10$ & $96.86(14)$ \\
\hline W9-O42-W11 & $95.65(13)$ \\
\hline $\mathrm{W} 10-\mathrm{O} 42-\mathrm{W} 11$ & $95.01(12)$ \\
\hline W11-O43-W9 & $114.98(17)$ \\
\hline $\mathrm{W} 10^{\mathrm{ii}}-\mathrm{O} 44-\mathrm{W} 12$ & $136.52(18)$ \\
\hline $\mathrm{W} 9-\mathrm{O} 45-\mathrm{W} 10$ & $119.05(18)$ \\
\hline $\mathrm{W} 10-\mathrm{O} 46-\mathrm{Na} 2$ & $166.1(2)$ \\
\hline $\mathrm{Na} 1-\mathrm{O} 49-\mathrm{Na} 2$ & $120.25(17)$ \\
\hline
\end{tabular}


supporting information

\begin{tabular}{llll}
$\mathrm{O} 38-\mathrm{W} 12-\mathrm{O} 31$ & $157.54(15)$ & $\mathrm{Na} 3-\mathrm{O} 52-\mathrm{Na} 2^{\mathrm{v}}$ & $113.31(16)$ \\
$\mathrm{O} 38-\mathrm{W} 12-\mathrm{O} 32$ & $86.74(14)$ & $\mathrm{Na} 4-\mathrm{O} 62-\mathrm{Na} 5$ & $112.24(17)$ \\
\hline
\end{tabular}

Symmetry codes: (i) $-x+1,-y+2,-z+2$; (ii) $-x,-y+1,-z+1$; (iii) $-x,-y+2,-z+1$; (iv) $-x+1,-y,-z+1$; (v) $-x+1,-y+1,-z+1$; (vi) $x, y-1, z$; (vii) $-x,-y+2$, $-z+2$; (viii) $-x,-y+3,-z+2$; (ix) $x, y+1, z$. 